\title{
Modeling and forecasting Daily stock Returns of Guaranty Trust Bank Nigeria PIc Using ARMA-GARCH Models, Persistence, Half-life Volatility and Backtesting
}

\author{
${ }^{1}$ Emenogu, G. Ngozi; ${ }^{2 *}$ Adenomon, Monday Osagie $\&{ }^{2}$ Nweze, Nwaze Obinna \\ 1. Department of Statistics, Federal Polytechnic, Bida, Niger State, Nigeria \\ 2. Department of Statistics, Nasarawa State University, Keffi, Nasarawa State, Nigeria \\ * Corresponding Authors: adenomonmo@nsuk.edu.ng;ngelive4jesus@gmail.com; \\ obininweze@yahoo.com +2347036990145; +2348036759112
}

\begin{abstract}
In financial time series modelling and forecasting, combining ARMA and GARCH models tend to produce superior and reliable models for volatility persistence, half-life volatility and backtesting (application of model in real life). In Nigeria, banking stocks are mostly traded because of its potential benefits to investors. This study modelled and forecasted the Guaranty Trust (GT) Bank daily stock returns from January $2^{\text {nd }} 2001$ to May $8^{\text {th }} 2017$ data set collected from a secondary source. The ARMA-GARCH models, persistence, half-life and backtesting were used to analysed the collected data using student $t$ and skewed student $t$ distributions, and the analyses are carried out $\mathrm{R}$ environment using rugard and performanceAnaytics Packages. The study revealed that using the lowest information criteria values only could be misleading rather we added the use of backtesing. The $\operatorname{ARMA}(1,1)-\mathrm{GARCH}(1,1)$ models fitted exhibited high persistency in the daily stock returns while the days it takes for mean-reverting of the models ranges from 5 days to 100 days but unfortunately the models failed backtesting. The results further revealed ARMA(1,1)-eGARCH $(2,2)$ model with student $t$ distribution provides a suitable model for evaluating the GT bank stock returns among the competing models while it takes less than 30 days for the persistence volatility to return back to its average value of the stock returns. This study recommended that researchers should adopt backtesting approach while fitting GARCH models while GT bank stocks investor should be assured that no matter the fluctuations in the stock market, the GT bank stock returns has the ability to returns to its mean price return.
\end{abstract}

Keywords: Returns, Stocks, Guaranty Trust (GT) Bank, Generalized Autoregressive Conditional Heteroskedasticity (GARCH), Persistence, Half-life, Volatility, Backtesting. 


\subsection{Introduction}

Autoregressive Moving Average (ARMA) and Autoregressive Integrated Moving Average (ARIMA) models are popular and excellent for modeling and forecasting univariate time series data as proposed by Box and Jenkins (1970), and its extension with exogenous variables as Autoregressive Integrated Moving Average with Explanatory Variable (ARIMAX) (Kongcharoen and Kruangpradit, 2013). These models are applied in almost all fields of endeavours such as engineering, geophysics, business, economics, finance, agriculture, medical sciences, social sciences, meteorology, quality control etc (Kirchgassner and Wolters, 2007; Adenomon, 2017a, Adenomon, 2017b; Cooray, 2008; Dobre and Alexandru, 2008; Gujarati, 2003; Adekeye and Aiyelabegan, 2006). The ARMA and ARIMA models are used to model conditional expectation of a process but in ARMA model, the conditional variance is constant. This means that ARMA model cannot capture process with time-varying conditional variance (volatility) which is mostly common with economic and financial data.

Actually, with economic and financial time series data, time-varying is more common than constant volatility, and accurate modeling of time volatility is of great importance in financial time series analysis (Ruppert, 2011). Financial time series contains uncertainty, volatility, excess kurtosis, high standard deviation, high skewness and sometimes non normality (Pedroni (2001); Grigoletto \& Lisi (2009); Emenogu and Adenomon, 2018; Emenogu et al. 2018). To model and capture properly the characteristics of financial time series models such as Auto-Regressive Conditional Heteroscedastic (ARCH), Generalized Auto-Regressive Conditional Heteroscedastic (GARCH), multivariate GARCH, Stochastic volatitlity (SV) and various variants of the models have been proposed to handle these characteristics of financial time series (Lawrance, 2003).

From the foregoing, considering the flexibility and simplicity of the ARMA model and the capability of the GARCH model to capture volatility in financial time series, combining the ARMA model with the GARCH model for the innovations, yielding the so-called ARMAGARCH model, provides the econometricians and financial analyst with a more flexible and yet tractable model that allows the model to capture the mean and variance components that is common with financial time series volatility (Lange, 2011; Panait and Slavescu, 2012) meaning that the ARMA-GARCH model will produced a more reliable estimates for financial analyst to 
take a better decision. This paper therefore investigates the persistence, half-life volatility and forecasting (Backtesting that is providing real life model) of daily stock returns of Guaranty Trust Bank, Nigeria plc using ARMA-GARCH Models.

Guaranty Trust (GT) Bank plc was incorporated as a limited liability company licensed to provide commercial and other banking services to the Nigerian public in 1990. The Bank commenced operations in February 1991, and has since then grown to become one of the most respected and service focused banks in Nigeria. Also, In September 1996, Guaranty Trust Bank plc became a publicly quoted company and won the Nigerian Stock Exchange President's Merit award that same year, and subsequently in the years 2000, 2003, 2005, 2006, 2007, 2008 and 2009. In February 2002, the Bank was granted a universal banking license and later appointed a settlement bank by the Central Bank of Nigeria (CBN) in 2003. In May 2011, The Bank successfully launched a US\$500 million bond - which bring about the first non-sovereign benchmark bond offering from sub-Saharan Africa (outside South Africa), to the international community. The highly successful offering, which matures in 2016, went further to show that the international finance community's believe in the GT Bank brand. The Bank's culture is tied to eight guiding principles called the Orange Rules; Simplicity, Professionalism, Service, Friendliness, Excellence, Trustworthiness, Social Responsibility and Innovation. With these features of GT Bank, the bank stands suitable for customers and investor both in Nigeria and abroad where the bank has branches (The European Financial Review, 2012).

\subsection{Brief literature on the persistence and half-life Volatility of Stocks Returns}

The volatility of asset returns is a measure of how much the returns fluctuates around its means (Marra, 2015). In addition, volatility is the purest measure of risk in financial markets and by this, it has becomes the expected price of uncertainty. A good volatility model and forecast help impact the public confidence significantly and by extension on the broader global economy. What comes to mind again is the persistence and half-life volatility of any given stock.

The persistence of financial stock is the extent to which events today have an efficient influence on the whole future history of a stochastic process, and as such is a central issue in financial time series, macroeconomic theory and policy (Caporale and Pittis, 2001). In a stationary GARCH process, the persistence volatility returns back to its means at the long term horizon and it is a rate calculated by the sum of GARCH and ARCH coefficients. And in many 
financial time series it is usually close to one (1) (Ahmed et al., 2018; Engle and Patton, 2001; Vosvrda, 2006). While on the other hand, the half-life of the volatility shocks measure the average time period for the volatility to return back to it mean value in the long run horizon (Ahmed et al., 2018; Sahai, 2016).

Engle and Patton (2001) examine the Dow Jones Industrial index from 23 August 1988 to 22 August 2000. Their result indicated that the volatility returns are quite persistent.

Magnus and Fosu (2006) modeled and forecasted the volatility of returns on Ghana Stock exchange using GARCH models. They found that presence of high level of persistence in the returns in the stock market.

Vosvrda (2006) compared empirical analysis of persistence and dependence patterns among capital market using univariate and multivariate measures. The results revealed that the univariate measure shows a low level of persistence while multivariate measure shows that the persistence change dependence on structure in different period of lags.

Panait and Slavescus (2012) investigated the volatility and persistence of seven Romanian companies traded on Bucharest Stock Exchange and three market indices from 19972012 using GARCH-in-Mean Models. They found out that persistency is more in the daily returns as compared to weekly and monthly series.

Emenike and Ani (2014) examined the nature of volatility of stock returns in the Nigerian banking sector using ARMA-GARCH models using data covering $3^{\text {rd }}$ January to December 2012. Their results revealed volatility persistence was high for the sample period they considered.

Usman et al. (2017) examined the performance of eleven competing GARCH models for fitting the rate of returns of monthly observations on the index returns series of the market over a period of January 1996 to December 2015. The overall results revealed increased volatility of the market returns.

Chu et al (2017) provided the first GARCH modeling of the seven most popular cryptocurrencies using twelve GARCH models fitted for each cryptocurrencies. Their work concluded IGARCH and GJR-GARCH models provided the best fits in terms of modeling of the volatility in the most popular and largest cryptocurrencies. 
Kuhe (2018) examined the volatility persistence and asymmetry with exogenous break in Nigerian stock market using data from $3^{\text {rd }}$ July 1999 to $12^{\text {th }}$ June 2017 using standard symmetric GARCH $(1,1)$, asymmetric EGARCH $(1,1)$ and GJR-GARCH $(1,1)$ models. The study revealed among other results a high persistence of shocks in the return series for the estimated models.

Ahmed et al. (2018) examined and compared the mean reversion phenomenon in developed and emerging stock markets, they employed data from $1^{\text {st }}$ January to $30^{\text {th }}$ June 2016 using GARCH $(1,1)$ model. There results revealed that South Korean market has the slowest mean reversion and thus has the highest half-life period while Pakistan stock exhibited fastest reverting process.

\subsection{Model Specification}

This study focuses on the ARMA-GARCH models that are robust for forecasting the volatility of financial time series data; so ARMA-GARCH model and some of its extensions are presented in this section.

ARMA-GARCH specification is employed to model the conditional mean and conditional variance (volatility) of any financial time series because of its superiority in modelling such series. GARCH models model conditional variances much as the conditional expectation by an ARMA model (Ruppert, 2011). Therefore ARMA model can be combined to any form of GARCH model.

The ARMA (p,q)-GARCH $(1,1)$ model can be specified as follows:

$$
\left.\begin{array}{l}
r_{t}=\sum_{i=1}^{p} \theta_{i} r_{t-i}+\sum_{j}^{q} \phi_{j} \varepsilon_{t-j}+\varepsilon_{t} \\
\varepsilon_{t} \sqrt{\sigma_{t}^{2} Z_{t}}, \quad Z_{t} \sim \mathrm{D}\left(0, \sigma_{\mathrm{t}}^{2}\right) \\
\sigma_{\mathrm{t}}^{2}=\omega+\alpha_{1} \varepsilon_{\mathrm{t}-1}^{2}+\beta_{1} \sigma_{\mathrm{t}-1}^{2}
\end{array}\right\}
$$

Where $r_{t}$ is the daily rate of return, $\theta$ is the $\operatorname{AR}(\mathrm{p})$ term in the mean equation in order to account for time dependence in returns, $\phi$ is the $\operatorname{MA}(q)$ term in the mean equation, $\varepsilon_{t}$ is the residual term in the mean equation, $\mathrm{Z}_{\mathrm{t}}$ is the standardized residual sequence of iid random variable with mean zero and variance as one while D represents distribution of the shock returns (Moshiri and Foroutan, 2006; Chen et al., 2013; Meitz and Saikkonen, 2011; Francq and Zakoian, 2004; Ling and McAleer, 2003; Koul and Ling, 2006; Zhu and Ling, 2011). 
Because of space we will discussion the follow family GARCH models below:

\subsection{Autoregressive Conditional Heteroskedasticity (ARCH) Family Model}

Every ARCH or GARCH family model requires two distinct specifications, namely: the mean and the variance equations (Atoi, 2014). The mean equation for a conditional heteroskedasticity in a return series, $y_{t}$ is given by

$$
y_{t}=E_{t-1}\left(y_{t}\right)+\varepsilon_{t}
$$

where

$$
\varepsilon_{t}=\phi_{t} \sigma_{t}
$$

The mean equation in equation (2) also applies to other GARCH family models. $E_{t-1}($.$) is the$ expected value conditional on information available at time $t-1$, while $\varepsilon_{t}$ is the error generated from the mean equation at time $\mathrm{t}$ and $\phi_{t}$ is the sequence of independent and identically distributed random variables with zero mean and unit variance.

The variance equation for an $\mathrm{ARCH}(\mathrm{p})$ model is given by

$$
\sigma_{t}^{2}=\omega+\alpha_{1} a_{t-1}^{2}+\ldots+\alpha_{p} a_{t-p}^{2}
$$

It can be seen in the equation that large values of the innovation of asset returns have bigger impact on the conditional variance because they are squared, which means that a large shock tends to follow another large shock and that is the same way the clusters of the volatility behave. So the $\mathrm{ARCH}(\mathrm{p})$ model becomes:

$$
a_{t}=\sigma_{t} \varepsilon_{t}, \quad \sigma_{t}^{2}=\omega+\alpha_{1} a_{t-1}^{2}+\ldots+\alpha_{p} a_{t-p}^{2}
$$

Where $\varepsilon_{t} \sim N(0,1)$ iid, $\omega>0$ and $\alpha_{i} \geq 0$ for $i>0$. In practice, $\varepsilon_{t}$ is assumed to follow the standard normal or a standardized student- $t$ distribution or a generalized error distribution (Tsay 2005).

\subsection{Asymmetric Power ARCH}

According to Rossi (2004), the asymmetric power ARCH model proposed by Ding, Engle \& Granger (1993) given below forms the basis for deriving the GARCH family models

Given that:

$$
\begin{aligned}
& r=\mu+a_{t}, \\
& \varepsilon_{t}=\sigma_{t} \varepsilon_{t}, \\
& \varepsilon_{t} \sim N(0,1)
\end{aligned}
$$




$$
\sigma_{t}^{\delta}=\omega+\sum_{i=1}^{p} \alpha_{i}\left(\left|a_{t-i}\right|-\gamma_{i} a_{t-i}\right)^{\delta}+\sum_{j=1}^{q} \beta_{j} \sigma_{t-j}^{\delta},
$$

where

$$
\begin{array}{ll}
\omega>0, & \delta \geq 0, \\
\alpha_{i} \geq 0 & i=1,2, \ldots, p \\
-1<\gamma_{i}<1 & i=1,2, \ldots, p \\
\beta_{j}>0 & j=1,2, \ldots, q
\end{array}
$$

This model imposes a Box-Cox transformation of the conditional standard deviation process and the asymmetric absolute residuals. The leverage effect is the asymmetric response of volatility to positive and negative "shocks".

\subsection{Standard $\operatorname{GARCH}(\mathbf{p}, q)$ Model:}

The mathematical model for the $\operatorname{sGARCH}(p, q)$ model is obtained from equation (5) by letting $\delta=2$ and $\gamma_{i}=0, i=1, \ldots, p$ to be:

$$
a_{t}=\sigma_{t} \varepsilon_{t}, \quad \sigma_{t}^{2}=\omega+\sum_{i=1}^{p} \alpha_{i} a_{t-i}^{2}+\sum_{j=1}^{q} \beta_{j} \sigma_{t-j}^{2}
$$

Where $a_{t}=r_{t}-\mu_{t}\left(r_{t}\right.$ is the continuously compounded log return series), and $\varepsilon_{t} \sim N(0,1)$ iid, the parameter $\alpha_{i}$ is the $\mathrm{ARCH}$ parameter and $\beta_{j}$ is the GARCH parameter, and $\omega>0, \alpha_{i} \geq 0, \beta_{j} \geq 0$, and $\sum_{i=1}^{\max (p, q)}\left(\alpha_{i}+\beta_{i}\right)<1$, (Rossi, 2004; Tsay, 2005 and Jiang, 2012).

The restriction on $\mathrm{ARCH}$ and GARCH parameters $\left(\alpha_{i}, \beta_{j}\right)$ suggests that the volatility $\left(a_{i}\right)$ is finite and that the conditional standard deviation $\left(\sigma_{i}\right)$ increases. It can be observed that if $\mathrm{q}=0$, then the model GARCH parameter $\left(\beta_{j}\right)$ becomes extinct and what is left is an ARCH(p) model. To expatiate on the properties of GARCH models, the following representation is necessary: Let $\eta_{t}=a_{t}^{2}-\sigma_{t}^{2}$ so that $\sigma_{t}^{2}=a_{t}^{2}-\eta_{t}$. By substituting $\sigma_{t-i}^{2}=a_{t-i}^{2}-\eta_{t-i},(i=0, \ldots, q)$ into Eq. (4), the GARCH model can be rewritten as

$$
a_{t}=\alpha_{0}+\sum_{i=1}^{\max (p, q)}\left(\alpha_{i}+\beta_{i}\right) a_{t-i}^{2}+\eta_{t}-\sum_{j=1}^{q} \beta_{j} \eta_{t-j},
$$

It can be seen that $\left\{\eta_{t}\right\}$ is a martingale difference series (i.e., $E\left(\eta_{t}\right)=0$ and 
$\operatorname{cov}\left(\eta_{t}, \eta_{t-j}\right)=0$, for $\left.j \geq 1\right)$. However, $\left\{\eta_{t}\right\}$ in general is not an iid sequence.

A GARCH model can be regarded as an application of the ARMA idea to the squared series $a_{t}^{2}$. Using the unconditional mean of an ARMA model, results in this

$$
\mathrm{E}\left(a_{t}^{2}\right)=\frac{\alpha_{0}}{1-\sum_{i=1}^{\max (p, q)}\left(\alpha_{i}+\beta_{i}\right)}
$$

provided that the denominator of the prior fraction is positive. (Tsay, 2005)

When $\mathrm{p}=1$ and $\mathrm{q}=1$, we have $\operatorname{GARCH}(1,1)$ model given by:

$$
\begin{aligned}
& a_{t}=\sigma_{t} \varepsilon_{t}, \\
& \sigma_{t}^{2}=\omega+\alpha_{1} a_{t-1}^{2}+\beta_{1} \sigma_{t-1}^{2},
\end{aligned}
$$

\subsection{GJR-GARCH(p, q) Model}

The Glosten-Jagannathan-Runkle GARCH (GJRGARCH) model, which is a model that attempts to address volatility clustering in an innovation process, is obtained by letting $\delta=2$.

When $\delta=2$ and $0 \leq \gamma_{i}<1$,

$$
\begin{aligned}
& \sigma_{t}^{2}=\omega+\sum_{i=1}^{p} \alpha_{i}\left(\left|\varepsilon_{t-i}\right|-\gamma_{i} \varepsilon_{t-i}\right)^{2}+\sum_{j=1}^{q} \beta_{j} \sigma_{t-j}^{2} \\
& =\omega+\sum_{i=1}^{p} \alpha_{i}\left(\left|\varepsilon_{t-i}\right|^{2}+\gamma_{i}^{2} \varepsilon_{t-1}^{2}-2 \gamma_{i}\left|\varepsilon_{t-i}\right| \varepsilon_{t-i}\right)+\sum_{j=1}^{q} \beta_{j} \sigma_{t-j}^{2} \\
& \sigma_{t}^{2}= \begin{cases}\omega+\sum_{i=1}^{p} \alpha_{i}^{2}\left(1+\gamma_{i}\right)^{2} \varepsilon_{t-i}^{2}+\sum_{j=1}^{q} \beta_{j} \sigma_{t-j}^{2}, & \varepsilon_{t-i}<0 \\
\omega+\sum_{i=1}^{p} \alpha_{i}\left(1-\gamma_{i}\right)^{2} \varepsilon_{t-i}^{2}+\sum_{j=1}^{q} \beta_{j} \sigma_{t-j}^{2}, & \varepsilon_{t-i}>0\end{cases}
\end{aligned}
$$

i.e; $\quad \sigma_{t}^{2}=\omega+\sum_{i=1}^{p} \alpha_{i}\left(1-\gamma_{i}\right)^{2} \varepsilon_{t-i}^{2}+\sum_{i=1}^{p} \alpha_{i}\left\{\left(1+\gamma_{i}\right)^{2}-\left(1-\gamma_{i}\right)^{2}\right\} S_{i}^{-} \varepsilon_{t-i}^{2}+\sum_{j=1}^{q} \beta_{j} \sigma_{t-j}^{2}$

$$
\sigma_{t}^{2}=\omega+\sum_{i=1}^{p} \alpha_{i}\left(1-\gamma_{i}\right)^{2} \varepsilon_{t-1}^{2}+\sum_{j=1}^{q} \beta_{j} \sigma_{t-1}^{2}+\sum_{i=1}^{p} 4 \alpha_{i} \gamma_{i} S_{i}^{-} \varepsilon_{t-i}^{2}
$$

where

$$
S_{i}^{-}=\left\{\begin{array}{l}
1 \text { if } \varepsilon_{t-i}<0 \\
0 \text { if } \varepsilon_{t-i} \geq 0
\end{array},\right.
$$

Now define 


$$
\alpha_{i}^{*}=\alpha_{i}\left(1-\gamma_{i}\right)^{2} \text { and } \gamma_{i}^{*}=4 \alpha_{i} \gamma_{i}
$$

then

$$
\sigma_{t}^{2}=\omega+\sum_{i=1}^{p} \alpha_{i}\left(1-\gamma_{i}\right)^{2} \varepsilon_{t-i}^{2}+\sum_{j=1}^{q} \beta_{j} \sigma_{t-i}^{2}+\sum_{i=1}^{p} \gamma_{i}^{*} S_{i}^{-} \varepsilon_{t-1}^{2}
$$

Which is the GJRGARCH model (Rossi, 2004).

But when $-1 \leq \gamma_{i}<0$,

Then recall Eq. (9)

$$
\begin{aligned}
& \sigma_{t}^{2}=\omega+\sum_{i=1}^{p} \alpha_{i}\left(\left|\varepsilon_{t-i}\right|-\gamma_{i} \varepsilon_{t-i}\right)^{2}+\sum_{j=1}^{q} \beta_{j} \sigma_{t-j}^{2} \\
& =\omega+\sum_{i=1}^{p} \alpha_{i}\left(\left|\varepsilon_{t-i}\right|^{2}+\gamma_{i}^{2} \varepsilon_{t-1}^{2}-2 \gamma_{i}\left|\varepsilon_{t-i}\right| \varepsilon_{t-i}\right)+\sum_{j=1}^{q} \beta_{j} \sigma_{t-j}^{2} \\
& \sigma_{t}^{2}= \begin{cases}\omega+\sum_{i=1}^{p} \alpha_{i}^{2}\left(1-\gamma_{i}\right)^{2} \varepsilon_{t-i}^{2}+\sum_{j=1}^{q} \beta_{j} \sigma_{t-j}^{2}, & \varepsilon_{t-i}>0 \\
\omega+\sum_{i=1}^{p} \alpha_{i}\left(1+\gamma_{i}\right)^{2} \varepsilon_{t-i}^{2}+\sum_{j=1}^{q} \beta_{j} \sigma_{t-j}^{2}, & \varepsilon_{t-i}<0\end{cases} \\
& \sigma_{t}^{2}=\omega+\sum_{i=1}^{p} \alpha_{i}\left(1+\gamma_{i}\right)^{2} \varepsilon_{t-i}^{2}+\sum_{j=1}^{q} \beta_{j} \sigma_{t-j}^{2}+\sum_{i=1}^{p} \alpha_{i}\left\{\left(1-\gamma_{i}\right)^{2}-\left(1+\gamma_{i}\right)^{2}\right\} S_{i}^{+} \varepsilon_{t-i}^{2} \\
& =\omega+\sum_{i=1}^{p} \alpha_{i}\left(1+\gamma_{i}\right)^{2} \varepsilon_{t-i}^{2}+\sum_{j=1}^{q} \beta_{j} \sigma_{t-j}^{2}+\sum_{i=1}^{p} \alpha_{i}\left\{1+\gamma_{i}^{2}-2 \gamma_{i}-1-\gamma_{i}^{2}-2 \gamma_{i}\right\} S_{i}^{+} \varepsilon_{t-i}^{2}
\end{aligned}
$$

Where

$$
S_{i}^{+}=\left\{\begin{array}{l}
1 \text { if } \varepsilon_{t-i}>0 \\
0 \text { if } \varepsilon_{t-i} \leq 0
\end{array}\right.
$$

also define

$$
\alpha_{i}^{*}=\alpha_{i}\left(1+\gamma_{i}\right)^{2} \text { and } \gamma_{i}^{*}=-4 \alpha_{i} \gamma_{i} \text {, }
$$

then

$$
\sigma_{t}^{2}=\omega+\sum_{i=1}^{p} \alpha_{i}^{*} \varepsilon_{t-i}^{2}+\sum_{j=1}^{q} \beta_{j} \sigma_{t-i}^{2}+\sum_{i=1}^{p} \gamma_{i}^{*} S_{i}^{+} \varepsilon_{t-1}^{2}
$$

which allows positive shocks to have a stronger effect on volatility than negative shocks (Rossi, 2004). But when $p=q=1$, the $\operatorname{GJRGARCH}(1,1)$ model will be written as 


$$
\sigma_{t}^{2}=\omega+\alpha \varepsilon_{t}^{2}+\gamma S_{i} \varepsilon_{t-1}^{2}+\beta \sigma_{t-1}^{2} .
$$

\subsection{IGARCH $(1,1)$ Model}

The integrated GARCH (IGARCH) models are unit- root GARCH models. The IGARCH $(1,1)$ model is specified in Tsay (2005) and Grek (2014) as

$$
a_{t}=\sigma_{t} \varepsilon_{t} ; \quad \sigma_{t}^{2}=\alpha_{0}+\beta_{1} \sigma_{t-1}^{2}+\left(1-\beta_{1}\right) a_{t-1}^{2}
$$

Where $\varepsilon_{t} \sim \mathrm{N}(0,1)$ iid, and $0<\beta_{1}<1$, Ali (2013) used $\alpha_{i}$ to denote $1-\beta_{i}$.

The model is also an exponential smoothing model for the $\left\{a_{t}^{2}\right\}$ series. To see this, rewrite the model as

$$
\begin{aligned}
\sigma_{t}^{2} & =\left(1-\beta_{1}\right) a_{t-1}^{2}+\beta_{1} \sigma_{t-1}^{2} \\
& =\left(1-\beta_{1}\right) a_{t-1}^{2}+\beta_{1}\left[(1-\beta) a_{t-2}^{2}+\beta_{1} \sigma_{t-2}^{2}\right] \\
& =\left(1-\beta_{1}\right) a_{t-1}^{2}+\left(1-\beta_{1}\right) \beta_{1} a_{t-2}^{2}+\beta_{1}^{2} \sigma_{t-2}^{2} .
\end{aligned}
$$

By repeated substitutions, we have

$$
\sigma_{t}^{2}=\left(1-\beta_{1}\right)\left(a_{t-1}^{2}+\beta_{1} a_{t-2}^{2}+\beta_{1}^{2} a_{t-3}^{3}+\cdots\right),
$$

which is the well-known exponential smoothing formation with $\beta_{1}$ being the discounting factor (Tsay, 2005).

\subsection{TGARCH(p, q) Model}

The Threshold GARCH model is another model used to handle leverage effects, and a $\operatorname{TGARCH}(\mathrm{p}, \mathrm{q})$ model is given by the following:

$$
\sigma_{t}^{2}=\alpha_{0}+\sum_{i=1}^{p}\left(\alpha_{i}+\gamma_{i} N_{t=i}\right) a_{t-i}^{2}+\sum_{j=1}^{q} \beta_{j} \sigma_{t-j}^{2},
$$

where $N_{t-i}$ is an indicator for negative $a_{t-i}$, that is,

$$
N_{t-i}=\left\{\begin{array}{l}
1 \text { if } a_{t-i}<0 \\
0 \text { if } a_{t-i} \geq 0
\end{array}\right.
$$

and $\alpha_{i}, \gamma_{i}$, and $\beta_{j}$ are nonnegative parameters satisfying conditions similar to those of GARCH models, (Tsay, 2005). When $p=1, q=1$, the TGARCH(1, 1) model becomes:

$$
\sigma_{t}^{2}=\omega+\left(\alpha+\gamma N_{t-1}\right) a_{t-1}^{2}+\beta \sigma_{t-1}^{2}
$$




\subsection{NGARCH $(p, q)$ Model}

The Nonlinear Generalized Autoregressive Conditional Heteroskedasticity (NGARCH) Model has been presented variously in literature by the following scholars: Hsieh \& Ritchken (2005), Lanne \& Saikkonen (2005), Malecka (2014) and Kononovicius \& Ruseckas (2015). The following model can be shown to represent all the presentations:

$$
h_{t}=\omega+\sum_{i=1}^{q} \alpha_{i} \varepsilon_{t-i}^{2}+\sum_{i=1}^{q} \gamma_{i} \varepsilon_{t-i}+\sum_{j=1}^{p} \beta_{j} h_{t-j}
$$

Where $h_{t}$ is the conditional variance, and $\omega, \beta$ and $\alpha$ satisfy $\omega>0, \beta \geq 0$ and $\alpha \geq 0$.

Which can also be written as

$$
\sigma_{t}=\omega+\sum_{i=1}^{q} \alpha_{i} \varepsilon_{t-i}^{2}+\sum_{i=1}^{q} \gamma_{i} \varepsilon_{t-i}+\sum_{j=1}^{p} \beta_{j} \sigma_{t-j}
$$

\subsection{The Exponential Generalized Autoregressive Conditional Heteroskedasticity (EGARCH) Model}

The EGARCH model was proposed by Nelson (1991) to overcome some weaknesses of the GARCH model in handling financial time series pointed out by Enocksson and Skoog(2012), In particular, to allow for asymmetric effects between positive and negative asset returns, he considered the weighted innovation

$$
g\left(\varepsilon_{t}\right)=\theta \varepsilon_{t}+\gamma\left[\left|\varepsilon_{t}\right|-E\left(\left|\varepsilon_{t}\right|\right)\right]
$$

where $\theta$ and $\gamma$ are real constants. Both $\varepsilon_{t}$ and $\left|\varepsilon_{t}\right|-E\left(\left|\varepsilon_{t}\right|\right)$ are zero-mean iid sequences with continuous distributions. Therefore, $E\left[g\left(\varepsilon_{t}\right)\right]=0$. The asymmetry of $g\left(\varepsilon_{t}\right)$ can easily be seen by rewriting it as

$$
g\left(\varepsilon_{t}\right)= \begin{cases}(\theta+\gamma) \varepsilon_{t}-\gamma E\left(\left|\varepsilon_{t}\right|\right) & \text { if } \varepsilon_{t} \geq 0 \\ (\theta-\gamma) \varepsilon_{t}-\gamma E\left(\left|\varepsilon_{t}\right|\right) & \text { if } \varepsilon_{t}<0\end{cases}
$$

$\operatorname{An} \operatorname{EGARCH}(m, s)$ model, according to Tsay (2005), Dhamija and Bhalla (2010), Jiang (2012), Ali (2013) and Grek (2014), can be written as

$$
a_{t}=\sigma_{t} \varepsilon_{t}, \quad \ln \left(\sigma_{t}^{2}\right)=\omega+\sum_{i=1}^{s} \alpha_{i} \frac{\left|a_{t-i}\right|+\theta_{i} a_{t-i}}{\sigma_{t-i}}+\sum_{j=1}^{m} \beta_{j} \ln \left(\sigma_{t-i}^{2}\right),
$$

Which specifically results in $\operatorname{EGARCH}(1,1)$ being written as 


$$
\begin{gathered}
a_{t}=\sigma_{t} \varepsilon_{t} \\
\ln \left(\sigma_{t}^{2}\right)=\omega+\alpha\left(\left[\left|a_{t-1}\right|-E\left(\left|a_{t-1}\right|\right)\right]\right)+\theta a_{t-1}+\beta \ln \left(\sigma_{t-1}^{2}\right)
\end{gathered}
$$

where $\left|a_{t-1}\right|-E\left(\left|a_{t-1}\right|\right)$ are iid and have mean zero. When the EGARCH model has a Gaussian distribution of error term, then $E\left(\left|\varepsilon_{t}\right|\right)=\sqrt{2 / \pi}$, which gives:

$$
\ln \left(\sigma_{t}^{2}\right)=\omega+\alpha\left(|| a_{t-1} \mid-\sqrt{2 / \pi}\right)+\theta a_{t-1}+\beta \ln \left(\sigma_{t-1}^{2}\right)
$$

\subsection{The Absolute Value GARCH (AVGARCH):}

An asymmetric GARCH (AGARCH), according to Ali (2013) is simply

$$
a_{t}=\sigma_{t} \varepsilon_{t} ; \sigma^{2}=\omega+\sum_{i=1}^{p} \alpha_{i}\left|\varepsilon_{t-i}-b\right|^{2}+\sum_{j=1}^{q} \beta_{j} \sigma_{t-j}^{2}
$$

While the absolute value generalized autoregressive conditional heteroskedasticity (AVGARCH) model is specified as:

$$
a_{t}=\sigma_{t} \varepsilon_{t} ; \quad \sigma^{2}=\omega+\sum_{i=1}^{p} \alpha_{i}\left(\left|\varepsilon_{t-i}+b\right|-c\left(\varepsilon_{t-i}+b\right)\right)^{2}+\sum_{j=1}^{q} \beta_{j} \sigma_{t-j}^{2}
$$

\subsection{Nonlinear (Asymmetric) GARCH, or N(A)GARCH or NAGARCH}

NAGARCH plays key role in option pricing with stochastic volatility because, as we shall see later on, NAGARCH allows you to derive closed-form expressions for European option prices in spite of the rich volatility dynamics. Because a NAGARCH may be written as

$$
\sigma_{t+1}^{2}=\omega+\alpha \sigma_{t}^{2}\left(z_{t}-\delta\right)^{2}+\beta \sigma_{t}^{2}
$$

And if $z_{t} \sim \operatorname{IIDN}(0,1), z_{t}$ is independent of $\sigma_{t}^{2}$ as $\sigma_{t}^{2}$ is only a function of an infinite number of past squared returns, it is possible to easily derive the long run, unconditional variance under NGARCH and the assumption of stationarity:

$$
\begin{aligned}
& E\left[\sigma_{t+1}^{2}\right]=\bar{\sigma}^{2}=\omega+\alpha E\left[\sigma_{t}^{2}\left(z_{t}-\delta\right)^{2}\right]+\beta E\left[\sigma_{t}^{2}\right] \\
& =\omega+\alpha E\left[\sigma_{t}^{2}\right] E\left(z_{t}^{2}+\delta^{2}-2 \delta z_{t}\right]+\beta E\left[\sigma_{t}^{2}\right] \\
& =\omega+\alpha \bar{\sigma}^{2}\left(1+\delta^{2}\right)+\beta \bar{\sigma}^{2}
\end{aligned}
$$

Where $\bar{\sigma}^{2}=E\left[\sigma_{t}^{2}\right]$ and $E\left[\sigma_{t}^{2}\right]=E\left[\sigma_{t+1}^{2}\right]$ because of stationary. Therefore 


$$
\bar{\sigma}^{2}\left[1-\alpha\left(1+\delta^{2}\right)+\beta\right]=\omega \Rightarrow \bar{\sigma}^{2}=\frac{\omega}{1-\alpha\left(1+\delta^{2}\right)+\beta}
$$

Which exists and positive if and only if $\alpha\left(1+\delta^{2}\right)+\beta<1$. This has two implications:

(i) The persistence index of a NAGARCH$(1,1)$ is $\alpha\left(1+\delta^{2}\right)+\beta$ and not simply $\alpha+\beta$;

(ii) a NAGARCH$(1,1)$ model is stationary if and only if $\alpha\left(1+\delta^{2}\right)+\beta<1$.

See details in Nelson (1991); Hall \& Yao (2003); Enders (2004); Christoffersen, et al. (2008) and Engle \& Rangel (2008).

\subsection{Persistence, Half-life Volatility and Backtesting}

\subsubsection{Persistence}

The low or high persistency in volatility exhibited by financial time series can be determined by the GARCH coefficients of a stationary GARCH model. The persistence of a GARCH model can be calculated as the sum of GARCH $\left(\beta_{1}\right)$ and $\mathrm{ARCH}\left(\alpha_{1}\right)$ coefficients that is $\alpha+\beta_{1}$. In most financial time series, it is very close to one (1) (Banerjee and Sarkar, 2006; Ahmed et al, 2018). Persistence could take the following conditions:

If $\alpha+\beta_{1}<1$ : The model ensures positive conditional variance as well as stationary.

If $\alpha+\beta_{1}=1$ : we have an exponential decay model, then the half-life becomes infinite. Meaning the model is strictly stationary.

If $\alpha+\beta_{1}>1$ : The GARCH model is said to be non-stationary, meaning that the volatility ultimately detonates toward the infinitude (Ahmed et al, 2018). In addition, the model shows that the conditional variance is unstable, unpredicted and the process is non-stationary (Kuhe, 2018).

\subsubsection{Half-Life Volatility}

Half-life volatility measures the mean reverting speed (average time) of a stock price or returns. The mathematical expression of half-life volatility is given as

$$
\text { Half }- \text { Life }=\frac{\ln (0.5)}{\ln \left(\alpha_{1}+\beta_{2}\right)}
$$

It can be noted that the value of $\alpha+\beta_{1}$ influences the mean reverting speed (Ahmed et al., 2018), which means that if the value of $\alpha+\beta_{1}$ is closer to one (1), then the volatility shocks of the half-life will be longer. 


\subsubsection{Backtesting}

Financial risk model evaluation or backtesting is an important part of the internal model's approach to market risk management as put out by Basle Committee on Banking Supervision (Christoffersen \& Pelletier, 2004). Backtesting is a statistical procedure where actual profits and losses are systematically compared to corresponding VaR estimates (Nieppola, 2009; Emenogu, et al., 2018). This study adopted Backstesting techniques of Christoffersen \& Pelletier, (2004); The test was implemented in $\mathrm{R}$ using rugarch package and this test considered both the unconditional (Kupiec) and conditional (Christoffersen) coverage tests for the correct number of exceedances (see details in Christoffersen(1998) and Christoffersen et al. (2001)).

The unconditional (Kupiec) test also refer to as POF-test (Proportion of failure) with its null hypothesis given as

$$
H_{0}: p=\hat{p}=\frac{y}{T}
$$

Here $\mathrm{y}$ is the number of exceptions and $\mathrm{T}$ is the number of observations and $\mathrm{k}$ is the confidence level. The test is given as

$$
L R_{P O F}=-2 \ln \left(\frac{(1-p)^{T-y} p^{y}}{\left[1-\left(\frac{y}{p}\right)^{T-y}\left(\frac{y}{T}\right)^{y}\right.}\right)
$$

Under the null hypothesis that the model is correct and $L R_{P O F}$ is asymptotically chi-squared $\left(\chi^{2}\right)$ distributed with degree of freedom as one (1). If the value of the $L R_{P O F}$ statistic is greater than the critical value (or $\mathrm{p}$-value $<0.01$ for $1 \%$ level of significant or $\mathrm{p}$-value $<0.05$ for $5 \%$ level of significant) the null hypothesis is rejected and the model then is inaccurate.

The Christoffersen's Interval Forecast Test combined the independence statistic with the Kupiec's POF test to obtained the joint test (Christoffersen, 1998; Nieppola, 2009). This test examined the properties of a good VaR model, the correct failure rate and independence of exceptions, that is condition coverage (cc). the conditional coverage (cc) is given as

$$
L R_{c c}=L R_{P O F}+L R_{\text {ind }}
$$

Where

$$
L R_{\text {ind }}=\sum_{i=2}^{n}\left[-2 \ln \left(\frac{p(1-p)^{u_{i}-1}}{\left(\frac{1}{u_{i}}\right)\left(1-\frac{1}{u_{i}}\right)^{u_{i}-1}}\right)\right]-2 \ln \left(\frac{p(1-p)^{u-1}}{\left(\frac{1}{u}\right)\left(1-\frac{1}{u}\right)^{u-1}}\right)
$$


Where $u_{i}$ is the time between exceptions I and i- 1 while $\mathrm{u}$ is the sum of $u_{i}$

If the value of the $L R_{c c}$ statistic is greater than the critical value (or p-value $<0.01$ for $1 \%$ level of significant or $\mathrm{p}$-value $<0.05$ for $5 \%$ level of significant) the null hypothesis is rejected and that leads to the rejection of the model.

\subsection{Distributions of GARCH models}

In this study we employed two innovations namely student $\mathrm{t}$ and skewed student $\mathrm{t}$ distributions they can account for excess kurtosis and non-normality in financial returns (Heracleous, 2003; Wilhelmsson, 2016; Kuhe, 2018).

The student $\mathrm{t}$ distribution is given as

$$
f(y)=\frac{\Gamma\left(\frac{v+1}{2}\right)}{\sqrt{v \pi} \Gamma\left(\frac{v}{2}\right)}\left(1+\frac{y^{2}}{v}\right)^{-\frac{(v+1)}{2}} ;-\infty<y<\infty
$$

The Skewed student $\mathrm{t}$ distribution is given as

$$
f(y ; \mu, \sigma, v, \lambda)=\left\{\begin{array}{l}
b c\left(1+\frac{1}{v-2}\left(\frac{b\left(\frac{y-\mu}{\sigma}\right)+a}{1-\lambda}\right)^{2}\right)^{-\frac{v+1}{2}}, \text { if } y<-\frac{a}{b} \\
b c\left(1+\frac{1}{v-2}\left(\frac{b\left(\frac{y-\mu}{\sigma}\right)+a}{1+\lambda}\right)^{2}\right)^{-\frac{v+1}{2}}, \text { if } y \geq-\frac{a}{b}
\end{array}\right.
$$

Where $v$ is the shape parameter with $2<v<\infty$ and $\lambda$ is the skewness parameter with $-1<\lambda<1$. The constants $\mathrm{a}, \mathrm{b}$ and $\mathrm{c}$ are given as

$$
a=4 \lambda c\left(\frac{v-2}{v-1}\right) ; b=1+3(\lambda)^{2}-a^{2} ; c=\frac{\Gamma\left(\frac{v+1}{2}\right)}{\sqrt{\pi(v-2) \Gamma\left(\frac{v}{2}\right)}}
$$

Where $\mu$ and $\sigma$ are the mean and the standard deviation of the skewed student $\mathrm{t}$ distribution respectively.

\subsection{Materials and Methods}

The data used in this study was collected from www.cashcraft.com under stock trend and analysis. Daily stock price for Guaranty Trust Bank Nigeria plc from January $2^{\text {nd }} 2001$ to May $8^{\text {th }}$ 2017 (a total of 4017 observations) was collected from the website. The returns was calculated using the formula below

$$
R_{t}=\ln P_{t}-\ln P_{t-1} .
$$

Then a total observation becomes 4016 . 


\subsection{Results}

The analyses of this study was carried in $\mathrm{R}$ environment using rugarch package by Ghalanos (2018) and PerformanceAnalytics package by Peterson et al.(2018). The section begins with the descriptive statistics of the daily stock price of GT Bank Nigeria, plc. Figures 1, 2, 3 and 4 presents the plot of the daily actual price of GT bank stock, the plot of the log Transform of the actual price of GT bank stock, the plot of log transformed of stock returns of GT Bank daily stock price and the plot of cleansed log transform of stock returns of GT Bank respectively.

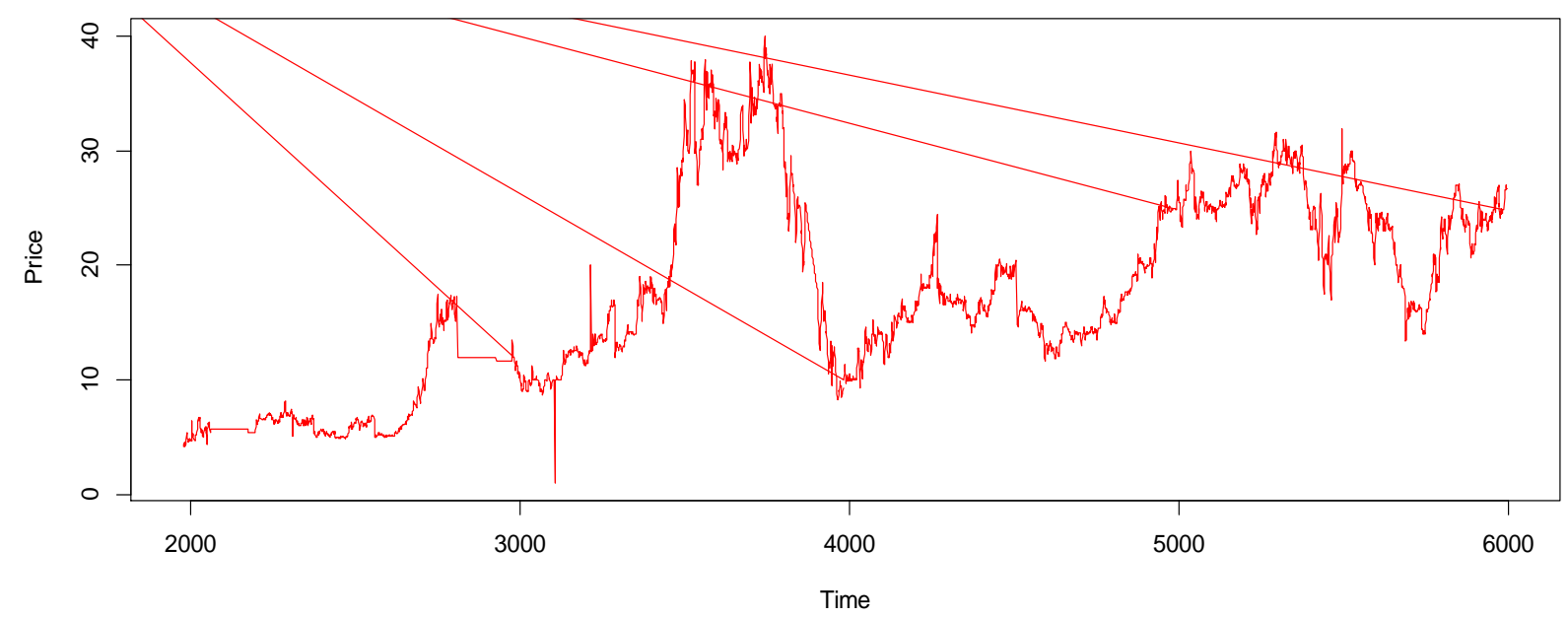

Fig. 1: Plot of the Actual price of GT bank stock

Figure 1 above presents the Actual price of the Guaranty Bank stock plc from January $2^{\text {nd }} 2001$ to May $8^{\text {th }} 2017$. The figure exhibited some trend. 


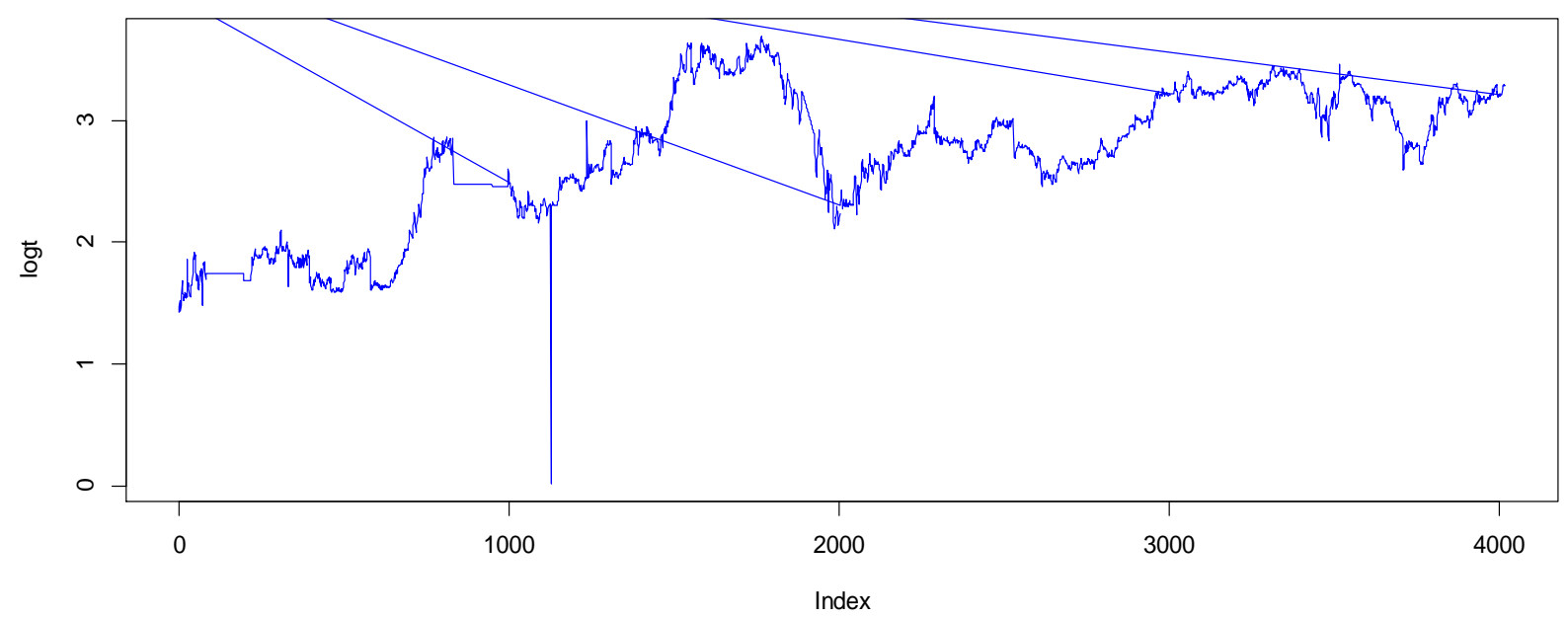

Fig. 2: Plot of the log Transform of the Actual price of GT bank stock Figure 2 above presents the log transform of the Actual price of the Guaranty Bank stock plc from January $2^{\text {nd }} 2001$ to May $8^{\text {th }} 2017$. The figure exhibited some pattern and achieved stability through transformation.

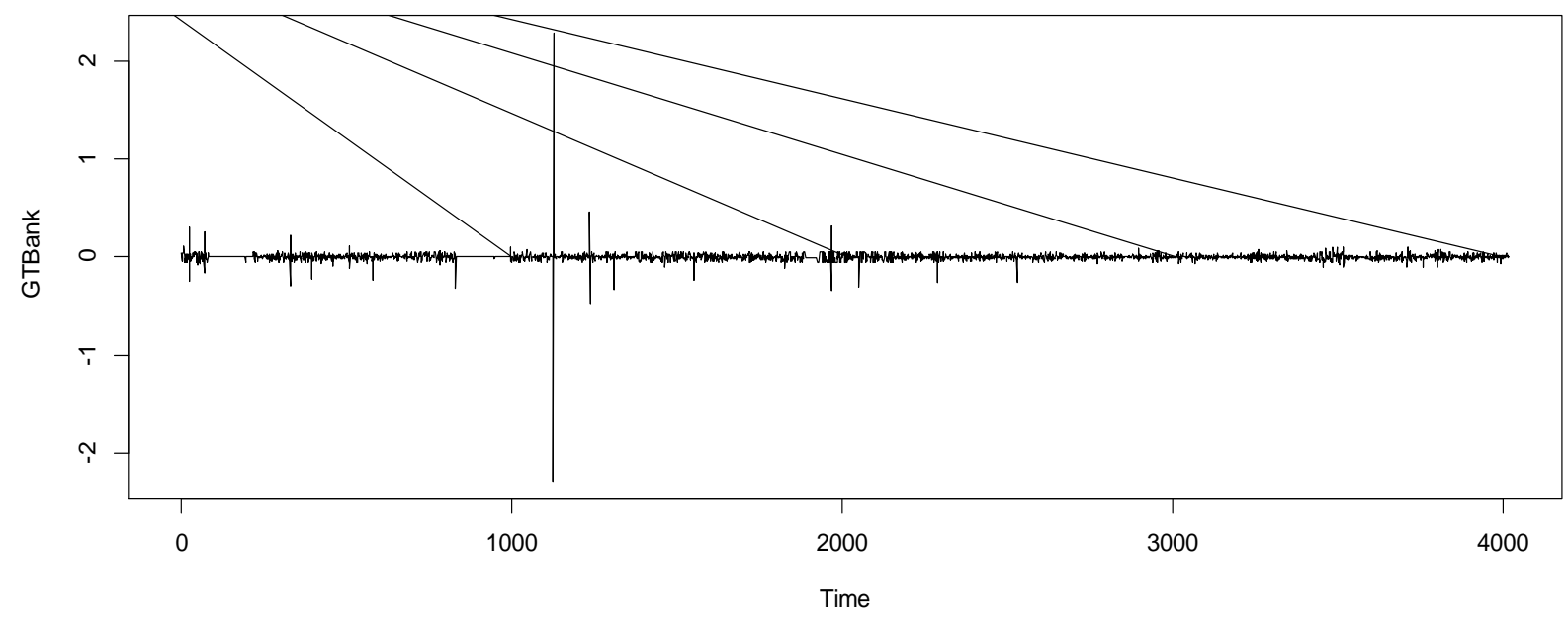

Fig 3: Plot of log transformed of stock returns of GT Bank

Figure 3 above presents the log transform of the stock returns of the Guaranty Bank stock plc from January $2^{\text {nd }} 2001$ to May $8^{\text {th }} 2017$. The figure actually exhibited the pattern of a typical financial time series that is volatility. 


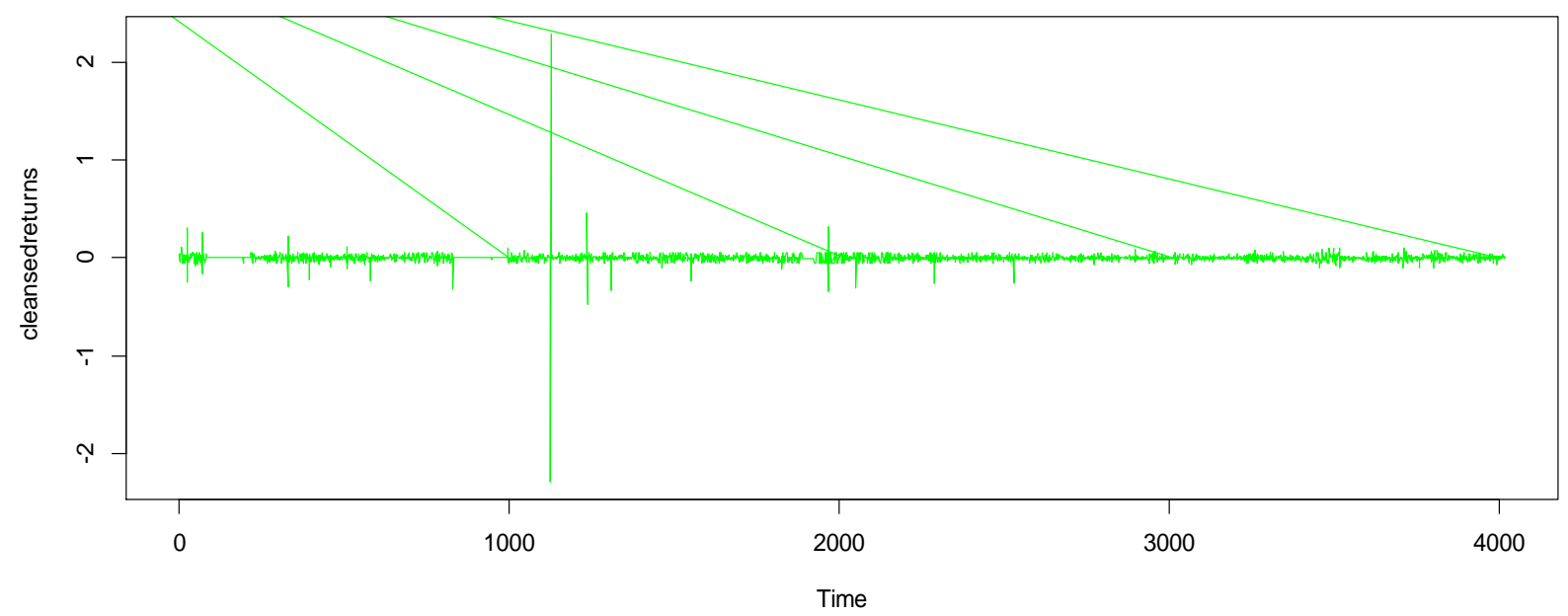

Fig 4: Plot of cleansed log transform of stock returns of GT Bank

Figure 4 above presents the cleansed log transform of the stock returns of the Guaranty Bank stock plc from January $2^{\text {nd }} 2001$ to May $8^{\text {th }} 2017$. His is done to remove the effects of possible outliers if any in the financial time series. The analysis of the financial time series in this study will be based on this cleansed series.

Table 1: Summary Statistics of Daily stock Returns of Guaranty Trust Bank Nigeria Plc

\begin{tabular}{|c|c|c|c|}
\hline Statistics & $\begin{array}{l}\text { Log of returns of Daily } \\
\text { Stock price }\end{array}$ & Actual Daily Stock Price & $\begin{array}{l}\text { Log transform of Daily } \\
\text { Actual Stock price }\end{array}$ \\
\hline Min & -2.28279 & 1.02 & 0.01980263 \\
\hline $\operatorname{Max}$ & 2.28279 & 39.98 & 3.688379 \\
\hline Median & 0 & 16.13 & 2.780681 \\
\hline Mean & 0.0004655802 & 17.32804 & 2.71587 \\
\hline Estimated sd & 0.05999086 & 8.334726 & 0.5533098 \\
\hline Estimated skewness & -0.2103046 & 0.3325078 & -0.5420392 \\
\hline Estimated kurtosis & 1049.868 & 2.242218 & 2.418776 \\
\hline Jarque-Bera & X-squared: & X-squared: 170.2176 & $x$-squared: 253.2493 \\
\hline Normality Test & $\begin{array}{l}182929273.4134 \\
\text { p Value: }<2.2 e-16\end{array}$ & p Value: $<2.2 e-16$ & p Value: $<2.2 e-16$ \\
\hline Number of & & & \\
\hline Observations & 4016 & 4017 & 4017 \\
\hline ARCH Test & $\begin{array}{l}\text { Chi-squared }=830.2 \\
\text { p-value }<2.2 e-16\end{array}$ & $\begin{array}{l}\text { Chi-squared }=3984.9 \\
\text { p-value }<2.2 e-16\end{array}$ & $\begin{array}{l}\text { Chi-squared }=3978.3 \\
\text { p-value }<2.2 e-16\end{array}$ \\
\hline $\begin{array}{l}\text { ADF-first difference } \\
\text { test }\end{array}$ & $\begin{array}{l}\text { test-statistic is: } \\
-91.9653 \\
\text { p-value: }<2.2 e-16\end{array}$ & $\begin{array}{l}\text { test-statistic is: } \\
-45.1483 \\
\text { p-value: }<2.2 e-16\end{array}$ & $\begin{array}{l}\text { test-statistic is: } \\
-61.079 \\
\text { p-value: }<2.2 e-16\end{array}$ \\
\hline
\end{tabular}


Table 1 above examined the characteristics of the financial time series used in this study. The actual stock price, the log transform of the stock price and the log transform of the stock returns exhibited the characteristics of a typical financial time series (i.e evidence of volatility) ( Abdulkareem \& Abdulkareem, 2016). The series exhibited large standard deviation, skewness and kurtosis. The series further exhibited non-normality using Jarque-Bera Statistic (pvalues<0.05) and shows the presence of ARCH effects (p-values<0.05), and all the type of series exhibited stationarity at first difference. In addition the averages of the stock series revealed positive values; this implies that the stock price is gaining. With this characteristics revealed above, GARCH and ARMA-GARCH models are appropriate in studying the volatility of the Guaranty Trust Bank stock returns.

Table 2: The Performance of the ARMA(1,1)-GARCH(1,1) Models using Information Criteria with respect to the distributions

\begin{tabular}{|l|l|l|l|}
\hline Models & $\begin{array}{l}\text { Information } \\
\text { Criteria }\end{array}$ & $\begin{array}{l}\text { Student } t \\
\text { distribution }\end{array}$ & $\begin{array}{l}\text { Skewed student } t \\
\text { distribution }\end{array}$ \\
\hline ARMA $(1,1)-$ GARCH $(1,1)$ & Akaike & -4.8457 & -4.8480 \\
& Bayes & -4.8347 & -4.8354 \\
& Shibata & -4.8457 & -4.8480 \\
& Hannan-Quinn & -4.8418 & -4.8435 \\
\hline ARMA $(1,1)-$ TGARCH $(1,1)$ & Akaike & -6.0027 & -6.0064 \\
& Bayes & -5.9917 & -5.9939 \\
& Shibata & -6.0027 & -6.0064 \\
& Hannan-Quinn & -5.9988 & -6.0020 \\
\hline ARMA (1,1)-NAGARCH $(1,1)$ & Akaike & -5.0575 & -5.0519 \\
& Bayes & -5.0466 & -5.0393 \\
& Shibata & -5.0575 & -5.0519 \\
& Hannan-Quinn & -5.0536 & -5.0474 \\
\hline ARMA (1, 1)-AVGARCH (1,1) & Akaike & -5.9990 & -5.9566 \\
& Bayes & -5.9864 & -5.925 \\
& Shibata & -5.9990 & -5.9566 \\
& Hannan-Quinn & -5.9945 & -5.9516 \\
\hline
\end{tabular}

In table 2 above, four competing models are compared using student $t$ distribution and skewed student $\mathrm{t}$ distribution. The following information criteria such as Akaike, Bayes, Shibata and Hannan-Quinn were used in selecting the preferred model. The results revealed ARMA(1,1)TGARCH(1,1) as preferred model with the least values of the information criteria using student $t$ and skewed student $t$ distributions. 
Table 3: The persistence and half-life volatility of the ARMA(1,1)-GARCH(1,1) models with respect to the distributions

\begin{tabular}{|c|c|c|c|}
\hline Models & Distributions & Persistence & Half-life (Days) \\
\hline \multirow[t]{2}{*}{$\operatorname{ARMA}(1,1)-\operatorname{eGARCH}(1,1)$} & $\begin{array}{l}\text { Student t } \\
\text { distribution }\end{array}$ & 0.8822875 & 5.534669 \\
\hline & $\begin{array}{l}\text { Skewed student } t \\
\text { distribution }\end{array}$ & 0.8853582 & 5.692593 \\
\hline \multirow[t]{2}{*}{$\operatorname{ARMA}(1,1)-\mathrm{TGARCH}(1,1)$} & $\begin{array}{l}\text { Student t } \\
\text { distribution }\end{array}$ & 0.9515151 & 13.94671 \\
\hline & $\begin{array}{l}\text { Skewed student } t \\
\text { distribution }\end{array}$ & 0.9503758 & 13.6184 \\
\hline \multirow[t]{2}{*}{$\operatorname{ARMA}(1,1)-\mathrm{NAGARCH}(1,1)$} & $\begin{array}{l}\text { Student } t \\
\text { distribution }\end{array}$ & 0.992533 & 92.48072 \\
\hline & $\begin{array}{l}\text { Skewed student } t \\
\text { distribution }\end{array}$ & 0.9855705 & 47.68934 \\
\hline \multirow[t]{2}{*}{$\operatorname{ARMA}(1,1)-\operatorname{AVGARCH}(1,1)$} & $\begin{array}{l}\text { Student } t \\
\text { distribution }\end{array}$ & 0.9481713 & 13.02416 \\
\hline & $\begin{array}{l}\text { Skewed student } t \\
\text { distribution }\end{array}$ & 0.939799 & 11.16372 \\
\hline
\end{tabular}

Evidence from persistence and half-life volatility in table 3 above shows that the Guaranty trust stock returns can be modeled and predicted since all the persistence values are all less than 1 (one). ARMA(1,1)-NAGARCH(1,1) exhibited the highest persistence and half-life volatility values while ARMA(1,1)-eGARCH $(1,1)$ exhibited the lowest persistence and half-life volatility values. For all the models, the days of mean-reverting ranges from 5 days to 95 days. 
Table 4: Backtesting of the ARMA(1,1)-GARCH(1,1): GARCH Roll Forecast (Backtest Length: 1016)

\begin{tabular}{|c|c|c|c|c|c|c|c|c|}
\hline \multirow{5}{*}{$\begin{array}{l}\text { Model } \\
\text { ARMA }(1,1)-\operatorname{eGARCH}(1,1)\end{array}$} & \multirow{3}{*}{$\begin{array}{l}\text { Distributions } \\
\text { Student } t\end{array}$} & \multirow{2}{*}{$\begin{array}{l}\text { Alpha } \\
\\
1 \%\end{array}$} & \multirow{2}{*}{$\begin{array}{l}\text { Expected } \\
\text { Exceed } \\
\\
10.2\end{array}$} & \multirow{2}{*}{$\begin{array}{l}\text { Actual } \\
\text { VaR } \\
\text { Exceed } \\
4\end{array}$} & \multicolumn{2}{|c|}{$\begin{array}{l}\text { Unconditional Coverage } \\
\text { (Kupiec) } \\
\mathrm{H}_{0} \text { : Correct Exceedances }\end{array}$} & \multicolumn{2}{|c|}{$\begin{array}{l}\text { Conditional Coverage (Christoffersen) } \\
\mathrm{H}_{0} \text { : Correct Exceedances and } \\
\text { independence of Failure }\end{array}$} \\
\hline & & & & & $\begin{array}{l}\text { LR.uc Statistic: } \\
\text { LR.uc Critical: } \\
\text { LR.uc p-value: } \\
\text { Decision: }\end{array}$ & $\begin{array}{r}4.9 \\
6.635 \\
0.027 \\
\text { Accept }\end{array}$ & $\begin{array}{l}\text { LR.CC Statistic: } \\
\text { LR.cC Critical: } \\
\text { LR.cC p-value: } \\
\text { Decision: }\end{array}$ & $\begin{array}{l}11.765 \\
9.21 \\
0.003 \\
\text { Reject }\end{array}$ \\
\hline & & $5 \%$ & 50.8 & 60 & $\begin{array}{l}\text { LR.uc Statistic: } \\
\text { LR.uc Critical: } \\
\text { LR.uc p-value: } \\
\text { Decision : }\end{array}$ & $\begin{array}{r}1.662 \\
3.841 \\
0.197 \\
\text { Accept }\end{array}$ & $\begin{array}{l}\text { LR.CC Statistic: } \\
\text { LR.CC Critical: } \\
\text { LR.cC p-value: } \\
\text { Decision: }\end{array}$ & $\begin{array}{l}2.267 \\
5.991 \\
0.322 \\
\text { Accept }\end{array}$ \\
\hline & $\begin{array}{l}\text { Skewed } \\
\text { student } t\end{array}$ & $1 \%$ & 10.2 & 6 & $\begin{array}{l}\text { LR.uc Statistic: } \\
\text { LR.uc Critical: } \\
\text { LR.uc p-value: } \\
\text { Decision: }\end{array}$ & $\begin{array}{r}2.017 \\
6.635 \\
0.156 \\
\text { Accept }\end{array}$ & $\begin{array}{l}\text { LR.CC Statistic: } \\
\text { LR.cC Critical: } \\
\text { LR.cC p-value: } \\
\text { Decision: }\end{array}$ & $\begin{array}{l}2.088 \\
9.21 \\
0.352 \\
\text { Accept }\end{array}$ \\
\hline & & $5 \%$ & 50.8 & 55 & $\begin{array}{l}\text { LR.uc Statistic: } \\
\text { LR.uc Critical: } \\
\text { LR.uc p-value: } \\
\text { Decision: }\end{array}$ & $\begin{array}{r}0.356 \\
3.841 \\
0.551 \\
\text { Accept } \\
\end{array}$ & $\begin{array}{l}\text { LR.CC Statistic: } \\
\text { LR.cC Critical: } \\
\text { LR.cC p-value: } \\
\text { Decision: }\end{array}$ & $\begin{array}{l}0.356 \\
5.991 \\
0.837 \\
\text { Accept }\end{array}$ \\
\hline \multirow[t]{4}{*}{$\operatorname{ARMA}(1,1)-\mathrm{TGARCH}(1,1)$} & \multirow[t]{2}{*}{ Student $t$} & $1 \%$ & 10.2 & 38 & $\begin{array}{l}\text { LR.uc Statistic: } \\
\text { LR.uc Critical: } \\
\text { LR.uc p-value: } \\
\text { Decision: }\end{array}$ & $\begin{array}{c}45.351 \\
6.635 \\
0 \\
\text { Reject }\end{array}$ & $\begin{array}{l}\text { LR.cC Statistic: } \\
\text { LR.cC Critical: } \\
\text { LR.cC p-value: } \\
\text { Decision: }\end{array}$ & $\begin{array}{l}45.578 \\
9.21 \\
0 \\
\text { Reject }\end{array}$ \\
\hline & & $5 \%$ & 50.8 & 96 & $\begin{array}{l}\text { LR.uc Statistic: } \\
\text { LR.uc Critical: } \\
\text { LR.uc p-value: } \\
\text { Decision: }\end{array}$ & $\begin{array}{c}33.949 \\
3.841 \\
0 \\
\text { Reject }\end{array}$ & $\begin{array}{l}\text { LR.CC Statistic: } \\
\text { LR.CC Critical: } \\
\text { LR.CC p-value: } \\
\text { Decision: }\end{array}$ & $\begin{array}{l}34.06 \\
5.991 \\
0 \\
\text { Reject }\end{array}$ \\
\hline & \multirow[t]{2}{*}{$\begin{array}{l}\text { Skewed } \\
\text { student } t\end{array}$} & $1 \%$ & 10.2 & 38 & $\begin{array}{l}\text { LR.uc Statistic: } \\
\text { LR.uc Critical: } \\
\text { LR.uc p-value: } \\
\text { Decision: }\end{array}$ & $\begin{array}{c}45.351 \\
6.635 \\
0 \\
\text { Reject }\end{array}$ & $\begin{array}{l}\text { LR.cC Statistic: } \\
\text { LR.cC Critical: } \\
\text { LR.cC p-value: } \\
\text { Decision: }\end{array}$ & $\begin{array}{l}45.578 \\
9.21 \\
0 \\
\text { Reject }\end{array}$ \\
\hline & & $5 \%$ & 50.8 & 96 & $\begin{array}{l}\text { LR.uc Statistic: } \\
\text { LR.uc Critical: } \\
\text { LR.uc p-value: } \\
\text { Decision: }\end{array}$ & $\begin{array}{c}33.949 \\
3.841 \\
0 \\
\text { Reject }\end{array}$ & $\begin{array}{l}\text { LR.cC Statistic: } \\
\text { LR.cC Critical: } \\
\text { LR.cC p-value: } \\
\text { Decision: }\end{array}$ & $\begin{array}{l}34.06 \\
5.991 \\
0 \\
\text { Reject }\end{array}$ \\
\hline $\operatorname{ARMA}(1,1)-$ & Student $t$ & $1 \%$ & 10.2 & 28 & $\begin{array}{l}\text { LR.uc Statistic: } \\
\text { LR.uc Critical: }\end{array}$ & $\begin{array}{r}21.408 \\
6.635\end{array}$ & $\begin{array}{ll}\text { LR.CC Statistic: } \\
\text { LR.CC Critical: }\end{array}$ & $\begin{array}{l}22.997 \\
9.21\end{array}$ \\
\hline
\end{tabular}




\begin{tabular}{|c|c|c|c|c|c|c|c|c|}
\hline \multirow[t]{4}{*}{$\operatorname{NAGARCH}(1,1)$} & & & & & $\begin{array}{l}\text { LR.uc p-value: } \\
\text { Decision: }\end{array}$ & $\begin{array}{c}0 \\
\text { Reject }\end{array}$ & $\begin{array}{l}\text { LR.cC p-value: } \\
\text { Decision: }\end{array}$ & $\begin{array}{l}0 \\
\text { Reject }\end{array}$ \\
\hline & & $5 \%$ & 50.8 & 90 & $\begin{array}{l}\text { LR.uc Statistic: } \\
\text { LR.uc Critical: } \\
\text { LR.uc p-value: } \\
\text { Decision: }\end{array}$ & $\begin{array}{c}26.158 \\
3.841 \\
0 \\
\text { Reject }\end{array}$ & $\begin{array}{l}\text { LR.CC Statistic: } \\
\text { LR.CC Critical: } \\
\text { LR.CC p-value: } \\
\text { Decision: }\end{array}$ & $\begin{array}{l}26.31 \\
5.991 \\
0 \\
\text { Reject }\end{array}$ \\
\hline & $\begin{array}{l}\text { Skewed } \\
\text { student } t\end{array}$ & $1 \%$ & 10.2 & 30 & $\begin{array}{l}\text { LR.uc Statistic: } \\
\text { LR.uc Critical: } \\
\text { LR.uc p-value: } \\
\text { Decision: }\end{array}$ & $\begin{array}{c}25.678 \\
6.635 \\
0 \\
\text { Reject }\end{array}$ & $\begin{array}{l}\text { LR.CC Statistic: } \\
\text { LR.CC Critical: } \\
\text { LR.CC p-value: } \\
\text { Decision: }\end{array}$ & $\begin{array}{l}27.506 \\
9.21 \\
0 \\
\text { Reject }\end{array}$ \\
\hline & & 5\% & 50.8 & 90 & $\begin{array}{l}\text { LR.uc Statistic: } \\
\text { LR.uc Critical: } \\
\text { LR.uc p-value: } \\
\text { Decision: }\end{array}$ & $\begin{array}{c}26.158 \\
3.841 \\
0 \\
\text { Reject }\end{array}$ & $\begin{array}{l}\text { LR.CC Statistic: } \\
\text { LR.CC Critical: } \\
\text { LR.CC p-value: } \\
\text { Decision: }\end{array}$ & $\begin{array}{l}26.31 \\
5.991 \\
0 \\
\text { Reject }\end{array}$ \\
\hline \multirow[t]{4}{*}{$\begin{array}{l}\operatorname{ARMA}(1,1)- \\
\operatorname{AVGARCH}(1,1)\end{array}$} & \multirow[t]{2}{*}{ Student $t$} & $1 \frac{\circ}{\circ}$ & 10.2 & 38 & $\begin{array}{l}\text { LR.uc Statistic: } \\
\text { LR.uc Critical: } \\
\text { LR.uc p-value: } \\
\text { Decision: }\end{array}$ & $\begin{array}{c}45.351 \\
6.635 \\
0 \\
\text { Reject }\end{array}$ & $\begin{array}{l}\text { LR.CC Statistic: } \\
\text { LR.CC Critical: } \\
\text { LR.CC p-value: } \\
\text { Decision: }\end{array}$ & $\begin{array}{l}45.578 \\
9.21 \\
0 \\
\text { Reject }\end{array}$ \\
\hline & & 5\% & 50.8 & 96 & $\begin{array}{l}\text { LR.uc Statistic: } \\
\text { LR.uc Critical: } \\
\text { LR.uc p-value: } \\
\text { Decision: }\end{array}$ & $\begin{array}{r}33.949 \\
3.841 \\
0 \\
\text { Reject }\end{array}$ & $\begin{array}{l}\text { LR.CC Statistic: } \\
\text { LR.CC Critical: } \\
\text { LR.CC p-value: } \\
\text { Decision: }\end{array}$ & $\begin{array}{l}34.419 \\
5.991 \\
0 \\
\text { Reject }\end{array}$ \\
\hline & \multirow[t]{2}{*}{$\begin{array}{l}\text { Skewed } \\
\text { student } t\end{array}$} & $1 \%$ & 10.2 & 37 & $\begin{array}{l}\text { LR.uc Statistic: } \\
\text { LR.uc Critical: } \\
\text { LR.uc p-value: } \\
\text { Decision: }\end{array}$ & $\begin{array}{c}42.685 \\
6.635 \\
0 \\
\text { Reject }\end{array}$ & $\begin{array}{l}\text { LR.CC Statistic: } \\
\text { LR.CC Critical: } \\
\text { LR.CC p-value: } \\
\text { Decision: }\end{array}$ & $\begin{array}{l}42.982 \\
9.21 \\
0 \\
\text { Reject }\end{array}$ \\
\hline & & 5\% & 50.8 & 97 & $\begin{array}{l}\text { LR.uc Statistic: } \\
\text { LR.uc Critical: } \\
\text { LR.uc p-value: } \\
\text { Decision: }\end{array}$ & $\begin{array}{c}35.33 \\
3.841 \\
0 \\
\text { Reject }\end{array}$ & $\begin{array}{l}\text { LR.CC Statistic: } \\
\text { LR.CC Critical: } \\
\text { LR.CC p-value: } \\
\text { Decision: }\end{array}$ & $\begin{array}{l}35.398 \\
5.991 \\
0 \\
\text { Reject }\end{array}$ \\
\hline
\end{tabular}


Backtesting approach is a means to select and use financial GARCH models for real life application. This approach revealed $\operatorname{ARMA}(1,1)-\operatorname{eGARCH}(1,1)$ as good model in respective of the distribution but only failed at $1 \%$ alpha level in student $\mathrm{t}$ distribution, this is supported by figures 5 to 8 at the appendix B while other models failed the Backtesting (figures omitted because of space). Furthermore, coefficients of the $\operatorname{ARMA}(1,1)$-eGARCH(1,1) model for both distributions (see Tables 8 and 9 at the appendix A) are more significant when compared to the other models (that is, $\operatorname{ARMA}(1,1)-\operatorname{TGARCH}(1,1) ; \operatorname{ARMA}(1,1)-\operatorname{NAGARCH}(1,1)$ and ARMA(1,1)-AVGARCH(1,1)) (see Tables 10 to 15 at the appendix A). These results led us to consider higher order GARCH model as ARMA(1,1)-GARCH(2,2) models.

Table 5: The Performance of the ARMA(1,1)-GARCH(2,2) Models using Information Criteria with respect to the distributions

\begin{tabular}{|c|c|c|c|}
\hline Models & Information Criteria & $\begin{array}{l}\text { Student } t \\
\text { distribution }\end{array}$ & $\begin{array}{l}\text { Skewed student } t \\
\text { distribution }\end{array}$ \\
\hline $\operatorname{ARMA}(1,1)-\operatorname{egARCH}(2,2)$ & $\begin{array}{l}\text { Akaike } \\
\text { Bayes } \\
\text { Shibata } \\
\text { Hannan-Quinn }\end{array}$ & $\begin{array}{l}-5.1904 \\
-5.1748 \\
-5.1904 \\
-5.1849\end{array}$ & $\begin{array}{l}-5.1334 \\
-5.1162 \\
-5.1335 \\
-5.1273 \\
\end{array}$ \\
\hline $\operatorname{ARMA}(1,1)-\mathrm{TGARCH}(2,2)$ & $\begin{array}{l}\text { Akaike } \\
\text { Bayes } \\
\text { Shibata } \\
\text { Hannan-Quinn }\end{array}$ & $\begin{array}{l}-5.9878 \\
-5.9721 \\
-5.9878 \\
-5.9822\end{array}$ & $\begin{array}{l}-5.9916 \\
-5.9743 \\
-5.9916 \\
-5.9855\end{array}$ \\
\hline $\operatorname{ARMA}(1,1)-\mathrm{NAGARCH}(2,2)$ & $\begin{array}{l}\text { Akaike } \\
\text { Bayes } \\
\text { Shibata } \\
\text { Hannan-Quinn }\end{array}$ & $\begin{array}{l}-5.0607 \\
-5.0450 \\
-5.0607 \\
-5.0551\end{array}$ & $\begin{array}{l}-5.0621 \\
-5.0449 \\
-5.0621 \\
-5.0560\end{array}$ \\
\hline $\operatorname{ARMA}(1,1)-\operatorname{AVGARCH}(2,2)$ & $\begin{array}{l}\text { Akaike } \\
\text { Bayes } \\
\text { Shibata } \\
\text { Hannan-Quinn }\end{array}$ & $\begin{array}{l}-6.0110 \\
-5.9922 \\
-6.0110 \\
-6.0043\end{array}$ & $\begin{array}{l}-5.9200 \\
-5.8996 \\
-5.9200 \\
-5.9127\end{array}$ \\
\hline
\end{tabular}

In table 5 above, four competing models are compared with respect to student $t$ distribution and skewed student $t$ distribution. The following information criteria such as Akaike, Bayes, Shibata and Hannan-Quinn were used in selecting the preferred model. The results revealed ARMA(1,1)$\operatorname{AVGARCH}(2,2)$ is preferred for student $t$ distribution and $\operatorname{ARMA}(1,1)-\operatorname{TGARCH}(2,2)$ model is preferred for skewed student $t$ distribution. 
Table 6: The persistence and half-life volatility of the ARMA(1,1)-GARCH(2,2) models with respect to the distributions

\begin{tabular}{|c|c|c|c|}
\hline Models & Distributions & Persistence & Half-life (Days) \\
\hline \multirow[t]{2}{*}{$\overline{\mathrm{ARMA}}(1,1)-\mathrm{eGARCH}(2,2)$} & $\begin{array}{l}\text { Student t } \\
\text { distribution }\end{array}$ & 0.9745043 & 26.83874 \\
\hline & $\begin{array}{l}\text { Skewed student } t \\
\text { distribution }\end{array}$ & 0.9576603 & 16.02202 \\
\hline \multirow[t]{2}{*}{$\operatorname{ARMA}(1,1)-\mathrm{TGARCH}(2,2)$} & $\begin{array}{l}\text { Student } t \\
\text { distribution }\end{array}$ & 0.9425471 & 11.71463 \\
\hline & $\begin{array}{l}\text { Skewed student } t \\
\text { distribution }\end{array}$ & 0.9399941 & 11.20117 \\
\hline \multirow[t]{2}{*}{$\operatorname{ARMA}(1,1)-\mathrm{NAGARCH}(2,2)$} & $\begin{array}{l}\text { Student t } \\
\text { distribution }\end{array}$ & 0.9810724 & 36.27328 \\
\hline & $\begin{array}{l}\text { Skewed student } t \\
\text { distribution }\end{array}$ & 0.986655 & 51.59337 \\
\hline \multirow[t]{2}{*}{$\operatorname{ARMA}(1,1)-\operatorname{AVGARCH}(2,2)$} & $\begin{array}{l}\text { Student t } \\
\text { distribution }\end{array}$ & 0.9876131 & 55.61095 \\
\hline & $\begin{array}{l}\text { Skewed student } t \\
\text { distribution }\end{array}$ & 0.9537416 & 14.63495 \\
\hline
\end{tabular}

Evidence from persistence and half-life volatility in table 6 above shows that the Guaranty trust stock returns can be modeled and predicted since all the persistence values are all less than 1 (one). ARMA(1,1)-AVGARCH(2,2) exhibited the highest persistence and half-life volatility values with respect to student $t$ distribution while $\operatorname{ARMA}(1,1)-\mathrm{NAGARCH}(2,2)$ exhibited the highest persistence and half-life volatility values with respect to skew student $t$ distribution. The $\operatorname{ARMA}(1,1)-\mathrm{TGARCH}(2,2)$ exhibited the lowest persistence and half-life volatility values for both distributions under consideration. For all the models, the days of mean-reverting ranges from 10 days to 60 days. 
Table 7: Backtesting of the ARMA(1,1)-GARCH(2,2): GARCH Roll Forecast (Backtest Length: 1016)

\begin{tabular}{|c|c|c|c|c|c|c|c|c|}
\hline \multirow{2}{*}{$\begin{array}{l}\text { Model } \\
\\
\operatorname{ARMA}(1,1)- \\
\operatorname{eGARCH}(2,2)\end{array}$} & \multirow{3}{*}{$\begin{array}{l}\text { Distributions } \\
\text { Student } t\end{array}$} & \multirow{2}{*}{$\begin{array}{l}\text { Alpha } \\
\\
1 \%\end{array}$} & \multirow{2}{*}{$\begin{array}{l}\text { Expected } \\
\text { Exceed } \\
10.2\end{array}$} & \multirow{2}{*}{$\begin{array}{l}\text { Actual } \\
\text { VaR } \\
\text { Exceed } \\
\\
9\end{array}$} & \multicolumn{2}{|c|}{$\begin{array}{l}\text { Unconditional Coverage (Kupiec) } \\
\mathrm{H}_{0} \text { : Correct Exceedances }\end{array}$} & \multicolumn{2}{|c|}{$\begin{array}{l}\text { Conditional Coverage } \\
\text { (Christoffersen) } \\
\mathrm{H}_{0} \text { : Correct Exceedances and } \\
\text { independence of Failure }\end{array}$} \\
\hline & & & & & $\begin{array}{l}\text { LR.uc Statistic: } \\
\text { LR.uc Critical: } \\
\text { LR.uc p-value: } \\
\text { Decision: }\end{array}$ & $\begin{array}{l}0.139 \\
6.635 \\
0.709 \\
\text { Accept }\end{array}$ & $\begin{array}{l}\text { LR.CC Statistic: } \\
\text { LR.CC Critical: } \\
\text { LR.cC p-value: } \\
\text { Decision: }\end{array}$ & $\begin{array}{l}0.3 \\
9.21 \\
0.861 \\
\text { Accept }\end{array}$ \\
\hline & & $5 \%$ & 50.8 & 58 & $\begin{array}{l}\text { LR.uc Statistic: } \\
\text { LR.uc Critical: } \\
\text { LR.uc p-value: } \\
\text { Decision: }\end{array}$ & $\begin{array}{l}1.029 \\
3.841 \\
0.31 \\
\text { Accept }\end{array}$ & $\begin{array}{l}\text { LR.CC Statistic: } \\
\text { LR.CC Critical: } \\
\text { LR.cC p-value: } \\
\text { Decision: }\end{array}$ & $\begin{array}{l}1.878 \\
5.991 \\
0.391 \\
\text { Accept }\end{array}$ \\
\hline & $\begin{array}{l}\text { Skewed } \\
\text { student } t\end{array}$ & $1 \%$ & 10.2 & 7 & $\begin{array}{l}\text { LR.uc Statistic: } \\
\text { LR.uc Critical: } \\
\text { LR.uc p-value: } \\
\text { Decision: }\end{array}$ & $\begin{array}{r}1.114 \\
6.635 \\
0.291 \\
\text { Accept }\end{array}$ & $\begin{array}{l}\text { LR. CC Statistic: } \\
\text { LR.cC Critical: } \\
\text { LR.cC p-value: } \\
\text { Decision: }\end{array}$ & $\begin{array}{l}1.211 \\
9.21 \\
0.546 \\
\text { Accept }\end{array}$ \\
\hline & & $5 \%$ & 50.8 & 66 & $\begin{array}{l}\text { LR.uc Statistic: } \\
\text { LR.uc Critical: } \\
\text { LR.uc p-value: } \\
\text { Decision: }\end{array}$ & $\begin{array}{r}4.393 \\
3.841 \\
0.036 \\
\text { Reject }\end{array}$ & $\begin{array}{l}\text { LR. CC Statistic: } \\
\text { LR.cC Critical: } \\
\text { LR.cC p-value: } \\
\text { Decision: }\end{array}$ & $\begin{array}{l}6.075 \\
5.991 \\
0.048 \\
\text { Reject }\end{array}$ \\
\hline \multirow[t]{4}{*}{$\begin{array}{l}\operatorname{ARMA}(1,1)- \\
\operatorname{TGARCH}(2,2)\end{array}$} & \multirow[t]{2}{*}{ student $t$} & $1 \frac{0}{0}$ & 10.2 & 31 & $\begin{array}{l}\text { LR.uc Statistic: } \\
\text { LR.uc Critical: } \\
\text { LR.uc p-value: } \\
\text { Decision: }\end{array}$ & $\begin{array}{l}27.918 \\
6.635 \\
0 \\
\text { Reject }\end{array}$ & $\begin{array}{l}\text { LR.cC Statistic: } \\
\text { LR.cC Critical: } \\
\text { LR.cC p-value: } \\
\text { Decision: }\end{array}$ & $\begin{array}{l}27.921 \\
9.21 \\
0 \\
\text { Reject }\end{array}$ \\
\hline & & $5 \%$ & 50.8 & 89 & $\begin{array}{l}\text { LR.uc Statistic: } \\
\text { LR.uc Critical: } \\
\text { LR.uc p-value: } \\
\text { Decision: }\end{array}$ & $\begin{array}{c}24.944 \\
3.841 \\
0 \\
\text { Reject }\end{array}$ & $\begin{array}{l}\text { LR.cC Statistic: } \\
\text { LR.cC Critical: } \\
\text { LR.cC p-value: } \\
\text { Decision: }\end{array}$ & $\begin{array}{l}25.637 \\
5.991 \\
0 \\
\text { Reject }\end{array}$ \\
\hline & \multirow[t]{2}{*}{$\begin{array}{l}\text { Skewed } \\
\text { student } t\end{array}$} & $1 \frac{0}{0}$ & 10.2 & 34 & $\begin{array}{l}\text { LR.uc Statistic: } \\
\text { LR.uc Critical: } \\
\text { LR.uc p-value: } \\
\text { Decision: }\end{array}$ & $\begin{array}{r}35.027 \\
6.635 \\
0 \\
\text { Reject }\end{array}$ & $\begin{array}{l}\text { LR.CC Statistic: } \\
\text { LR.cC Critical: } \\
\text { LR.cC p-value: } \\
\text { Decision: }\end{array}$ & $\begin{array}{l}35.046 \\
9.21 \\
0 \\
\text { Reject }\end{array}$ \\
\hline & & $5 \%$ & 50.8 & 90 & $\begin{array}{l}\text { LR.uc Statistic: } \\
\text { LR.uc Critical: } \\
\text { LR.uc p-value: } \\
\text { Decision: }\end{array}$ & $\begin{array}{l}26.158 \\
3.841 \\
0 \\
\text { Reject }\end{array}$ & $\begin{array}{l}\text { LR.CC Statistic: } \\
\text { LR.cC Critical: } \\
\text { LR.cC p-value: } \\
\text { Decision: }\end{array}$ & $\begin{array}{l}26.736 \\
5.991 \\
0 \\
\text { Reject }\end{array}$ \\
\hline $\operatorname{ARMA}(1,1)-$ & Student $t$ & $1 \%$ & 10.2 & 41 & $\begin{array}{l}\text { LR.uC Statistic: } \\
\text { LR.uc Critical: }\end{array}$ & $\begin{array}{r}53.675 \\
6.635\end{array}$ & $\begin{array}{l}\text { LR.CC Statistic: } \\
\text { LR.CC Critical: }\end{array}$ & $\begin{array}{l}53.748 \\
9.21\end{array}$ \\
\hline
\end{tabular}




\begin{tabular}{|c|c|c|c|c|c|c|c|c|}
\hline \multirow[t]{4}{*}{ NAGARCH $(2,2)$} & & & & & $\begin{array}{l}\text { LR.uc p-value: } \\
\text { Reject Null: }\end{array}$ & $\begin{array}{l}0 \\
\text { YES }\end{array}$ & $\begin{array}{l}\text { LR.cC p-value: } \\
\text { Decision: }\end{array}$ & $\begin{array}{c}0 \\
\text { Reject }\end{array}$ \\
\hline & & $5 \%$ & 50.8 & 109 & $\begin{array}{l}\text { LR.uc Statistic: } \\
\text { LR.uc Critical: } \\
\text { LR.uc p-value: } \\
\text { Decision: }\end{array}$ & $\begin{array}{l}53.615 \\
3.841 \\
0 \\
\text { Reject }\end{array}$ & $\begin{array}{l}\text { LR.CC Statistic: } \\
\text { LR.CC Critical: } \\
\text { LR.cC p-value: } \\
\text { Decision: }\end{array}$ & $\begin{array}{l}53.624 \\
5.991 \\
0 \\
\text { Reject }\end{array}$ \\
\hline & \multirow[t]{2}{*}{$\begin{array}{l}\text { Skewed } \\
\text { student } t\end{array}$} & $1 \%$ & 10.2 & 30 & $\begin{array}{l}\text { LR.uc Statistic: } \\
\text { LR.uc Critical: } \\
\text { LR.uc p-value: } \\
\text { Decision: }\end{array}$ & $\begin{array}{r}25.678 \\
6.635 \\
0 \\
\text { Reject }\end{array}$ & $\begin{array}{l}\text { LR.CC Statistic: } \\
\text { LR.cC Critical: } \\
\text { LR.cC p-value: } \\
\text { Decision: }\end{array}$ & $\begin{array}{l}25.693 \\
9.21 \\
0 \\
\text { Reject }\end{array}$ \\
\hline & & $5 \%$ & 50.8 & 92 & $\begin{array}{l}\text { LR.uc Statistic: } \\
\text { LR.uc Critical: } \\
\text { LR.uc p-value: } \\
\text { Decision: }\end{array}$ & $\begin{array}{l}28.66 \\
3.841 \\
0 \\
\text { Reject }\end{array}$ & $\begin{array}{l}\text { LR.cC Statistic: } \\
\text { LR.cC Critical: } \\
\text { LR.cC p-value: } \\
\text { Decision: }\end{array}$ & $\begin{array}{l}30.415 \\
5.991 \\
0 \\
\text { Reject }\end{array}$ \\
\hline \multirow[t]{4}{*}{$\begin{array}{l}\operatorname{ARMA}(1,1)- \\
\operatorname{AVGARCH}(2,2)\end{array}$} & \multirow[t]{2}{*}{ Student $t$} & $1 \%$ & 10.2 & 34 & $\begin{array}{l}\text { LR.uc Statistic: } \\
\text { LR.uc Critical: } \\
\text { LR.uc p-value: } \\
\text { Decision: }\end{array}$ & $\begin{array}{r}35.027 \\
6.635 \\
0 \\
\text { Reject }\end{array}$ & $\begin{array}{l}\text { LR.cC Statistic: } \\
\text { LR.cC Critical: } \\
\text { LR.cC p-value: } \\
\text { Decision: }\end{array}$ & $\begin{array}{l}35.603 \\
9.21 \\
0 \\
\text { Reject }\end{array}$ \\
\hline & & $5 \%$ & 50.8 & 86 & $\begin{array}{l}\text { LR.uc Statistic: } \\
\text { LR.uc Critical: } \\
\text { LR.uc p-value: } \\
\text { Decision: }\end{array}$ & $\begin{array}{l}21.449 \\
3.841 \\
0 \\
\text { Reject }\end{array}$ & $\begin{array}{l}\text { LR.CC Statistic: } \\
\text { LR.CC Critical: } \\
\text { LR.CC p-value: } \\
\text { Decision: }\end{array}$ & $\begin{array}{l}22.551 \\
5.991 \\
0 \\
\text { Reject }\end{array}$ \\
\hline & \multirow[t]{2}{*}{$\begin{array}{l}\text { Skewed } \\
\text { student } t\end{array}$} & $1 \%$ & 10.2 & 36 & $\begin{array}{l}\text { LR.uc Statistic: } \\
\text { LR.uc Critical: } \\
\text { LR.uc p-value: } \\
\text { Decision: }\end{array}$ & $\begin{array}{r}40.074 \\
6.635 \\
0 \\
\text { Reject }\end{array}$ & $\begin{array}{l}\text { LR.CC Statistic: } \\
\text { LR.CC Critical: } \\
\text { LR.cC p-value: } \\
\text { Decision: }\end{array}$ & $\begin{array}{l}40.143 \\
9.21 \\
0 \\
\text { Reject }\end{array}$ \\
\hline & & $5 \%$ & 50.8 & 89 & $\begin{array}{l}\text { LR.uc Statistic: } \\
\text { LR.uc Critical: } \\
\text { LR.uc p-value: } \\
\text { Decision: }\end{array}$ & $\begin{array}{l}24.944 \\
3.841 \\
0 \\
\text { Reject }\end{array}$ & $\begin{array}{l}\text { LR.cC Statistic: } \\
\text { LR.cC Critical: } \\
\text { LR.cC p-value: } \\
\text { Decision: }\end{array}$ & $\begin{array}{l}26.371 \\
5.991 \\
0 \\
\text { Reject }\end{array}$ \\
\hline
\end{tabular}


Backtesting approach revealed ARMA(1,1)-eGARCH(2,2) as good model in respective of the distribution at $1 \%$ and $5 \%$ alpha levels, this is supported by figures 9 to 12 at the appendix B while other models failed the Backtesting (figures omitted because of space). Furthermore, coefficients of the ARMA(1,1)-eGARCH(2,2) model for both distributions are more significant (see Tables 16 and 17 at appendix A) when compared to the other models (that is, ARMA(1,1)TGARCH(2,2); ARMA(1,1)-NAGARCH(2,2) and $\operatorname{ARMA}(1,1)-\operatorname{AVGARCH}(2,2))$ see Tables 18 to 23 .

\section{$5.1 \quad$ Discussion of Results}

The log transform of the Guaranty Trust Bank stock returns exhibited the characteristics of a typical financial time series (i.e evidence of volatility) (Abdulkareem \& Abdulkareem, 2016) as shown in Table 1. The series exhibited large standard deviation, skewness and kurtosis. The series further exhibited non-normality using Jarque-Bera Statistic (p-values<0.05), shows the presence of ARCH effects (p-values $<0.05$ ) and the series exhibited stationarity at first difference. In addition the average value of the returns revealed a positive value which implies that the stock price is gaining (Kuhe, 2018). With these characteristics of the stock returns, the GARCH and ARMA-GARCH models are appropriate in studying the volatility of the Guaranty Trust Bank stock returns (Emenike \& Ani, 2014; Ahmed et al, 2018).

In table 2, the four competing models were compared using student $\mathrm{t}$ distribution and skewed student $\mathrm{t}$ distribution. The following information criteria such as Akaike, Bayes, Shibata and Hannan-Quinn were used to select the preferred model. The results revealed ARMA(1,1)TGARCH(1,1) as preferred model with the least values of the information criteria for both student $\mathrm{t}$ and skew student $\mathrm{t}$ distributions.

Evidence from persistence and half-life volatility in table 3 shows that the Guaranty trust stock returns can be modeled and predicted since all the persistence values are all less than 1 (one). This also means that the models ensures positive conditional variance as well as stationary (Banerjee and Sarkar, 2006; Ahmed et al, 2018). The ARMA(1,1)-NAGARCH(1,1) exhibited the highest persistence and half-life volatility values while $\operatorname{ARMA}(1,1)$-eGARCH$(1,1)$ exhibited the lowest persistence and half-life volatility values for both distributions. For all the models, the days of mean-reverting ranges from 5 days to 95 days (that is within three (3) months). 
Backtesting approach is a means to select and use financial GARCH models for real life application. This approach revealed $\operatorname{ARMA}(1,1)-\operatorname{eGARCH}(1,1)$ as good model for both distributions but only failed the Conditional Coverage (Christoffersen), this is Correct Exceedances and independence of Failure at $1 \%$ alpha level in student $\mathrm{t}$ distribution, this is supported by figures 5 to 8 at the appendix B. This contradicts the results from the information criteria that selected $\operatorname{ARMA}(1,1)-\operatorname{TGARCH}(1,1)$ as the preferred model. This suggests model should not be selected by information criteria only but should be selected in addition by how significant is the coefficients of the model and possibly by backtesting approach (Christoffersen, 1998; Nieppola, 2009). The other models under considerations failed the Backtesting (figures omitted because of space). Furthermore, coefficients of the $\operatorname{ARMA}(1,1)$-eGARCH(1,1) model for both distributions (see Tables 8 and 9 in Appendix A) are more significant when compared to the other models (that is, $\operatorname{ARMA}(1,1)-\mathrm{TGARCH}(1,1) ; \operatorname{ARMA}(1,1)-\operatorname{NAGARCH}(1,1)$ and ARMA(1,1)-AVGARCH(1,1)) (see Tables 10 to 15 in Appendix). These results led us to consider higher order GARCH model as $\operatorname{ARMA}(1,1)-\operatorname{GARCH}(2,2)$ models which follows the works Starica (2003) and, Hansen and Lunde (2005) that revealed that the GARCH(1,1) was clearly inferiors to models that can accommodate a leverage effect. But our results contradicts the work of Namugaya et al (2014) that GARCH(1.1) outperformed the higher order of GARCH models, this could be because their work did not consider how good is their model.

In table 5, the four competing models of higher order were compared with respect to student $\mathrm{t}$ distribution and skewed student $t$ distribution. The following information criteria such as Akaike, Bayes, Shibata and Hannan-Quinn were employed to select the preferred model. The results revealed $\operatorname{ARMA}(1,1)$-AVGARCH(2,2) is preferred for student $\mathrm{t}$ distribution and $\operatorname{ARMA}(1,1)$ TGARCH(2,2)model is preferred for skew student $t$ distribution.

Evidence from persistence and half-life volatility in table 6 shows that the Guaranty trust stock returns can be modeled and predicted since all the persistence values are all less than 1 (one). This also means that the models ensures positive conditional variance as well as stationary(Banerjee and Sarkar, 2006; Ahmed et al, 2018). ARMA(1,1)-AVGARCH(2,2) exhibited the highest persistence and half-life volatility values with respect to student $t$ distribution while $\operatorname{ARMA}(1,1)-\mathrm{NAGARCH}(2,2)$ exhibited the highest persistence and half-life volatility values with respect to skewed student $t$ distribution. The ARMA(1,1)-TGARCH(2,2) 
exhibited the lowest persistence and half-life volatility values for both distributions under consideration. For all the models, the days of mean-reverting ranges from 10 days to 60 days. Backtesting approach revealed $\operatorname{ARMA}(1,1)-\mathrm{eGARCH}(2,2)$ in Table 7 as good model in respective of the distribution at $1 \%$ and $5 \%$ alpha levels, this is supported by figures 9 to 12 at the appendix B while other models failed the Backtesting (figures omitted because of space). Furthermore, coefficients of the ARMA(1,1)-eGARCH(2,2) model for both distributions (see Tables 16 and 17 in Appendix A) are more significant when compared to the other models (that is, $\operatorname{ARMA}(1,1)-\mathrm{TGARCH}(2,2) ; \quad \operatorname{ARMA}(1,1)-\mathrm{NAGARCH}(2,2)$ and $\operatorname{ARMA}(1,1)-$ AVGARCH(2,2)) (see Tables 18 to 23 in Appendix A). As mention earlier, ARMA(1,1)eGARCH(2,2) was selected because it completely passed the backtesting though ARMA(1,1)$\operatorname{AVGARCH}(2,2)$ was selected by information criteria. This suggests model should not be selected by information criteria only but should be selected in addition by how significant is the coefficients of the model and possibly by backtesting approach(Christoffersen, 1998; Nieppola, 2009). Lastly, in all the models considered, there were no ARCH effects in the residuals of the estimated models.

\subsection{Conclusion and recommendations}

The Guaranty Trust (GT) Bank's culture is tied to eight guiding principles called the Orange Rules; Simplicity, Professionalism, Service, Friendliness, Excellence, Trustworthiness, Social Responsibility and Innovation. With these features of GT Bank, the bank stands suitable for customers and investor both in Nigeria and abroad where the bank has branches. In fact, the US Embassy used GT Bank as a means paying for Visa application in Nigeria. The GT bank stock investors and customers should be assured as GT bank is a stock in emerging market to invest in (Ahmed et al., 2018). This is true because all the models considered ensures positive conditional variance as well as stationary (Banerjee and Sarkar, 2006; Ahmed et al, 2018).

The study revealed that using the lowest information criteria values only could not be enough to select preferred GARCH model rather we should add the use of backtesing. The models fitted exhibited high persistency in the daily stock returns while the days it takes for mean-reverting of the models ranges from 5 days to 100 days. The results further revealed ARMA(1,1)-GARCH $(2,2)$ model with student $\mathrm{t}$ distribution provides a suitable model for evaluating the GT bank 
stock returns among the competing models while it takes less than 30 days for the persistence volatility to return back to its average value of the stock returns.

This study recommended that researchers should adopt backtesting approach while fitting GARCH models while GT bank stocks investor should be assured that no matter the fluctuations in the stock market, the GT bank stock returns has the ability to returns to its mean price returns.

\section{References}

Abdulkareem, A. and Abdulkareem, K. A.(2016): Analyzing Oil Price-Macroeconomic Volatility in Nigeria. CBN Journal of Applied Statistics, 7(1a):1-22.

Adekeye KS \& Aiyelabegan AB 2006. Fitting An ARIMA Model to Experimental Data. Nigerian Statistical Association (NSA) Conference Proceedings, pp. 6572.

Adenomon, M. O. (2017a): Introduction to Univariate and Multivariate Time Series Analysis with Examples in R. Nigeria: University Press Plc.

Adenomon, M. O.(2017b): Modelling and Forecasting Unemployment Rates in Nigeria Using ARIMA Model. FUW Trends in Science \& Technology Journal, 2(1B):525-531.

Ahmed, R. R.; Vveinhardt, J.; Streimikiene, D. and Channar, Z. A.(2018): Mean Reversion in International Markets: Evidence from GARCH and Half-Life Volatility Models. Economic Research, 31(1):1198-1217.

Ali, G. (2013) "EGARCH, GJR-GARCH, TGARCH, AVGARCH, NGARCH, IGARCH, and APARCH Models for Pathogens at Marine Recreational Sites". Journal of Statistical and Econometric Methods, 2 (3): 57-73.

Atoi, N. V. (2014) “Testing volatility in Nigeria stock market using GARCH models". CBN Journal of Applied Statistics, 5: 65-93.

Banerjee, A., \& Sarkar, S. (2006). Modeling daily volatility of the Indian stock market using intraday data. Working Paper No. 588, IIM, Calcutta. Retrieved March 1, 2017, from http://www.iimcal. ac.in/res/upd\%5CWPS\%20588.pdf

Box GEP and Jenkins GM 1970. Time Series Analysis, Forecasting and Control. San Francisco: Holden-Day.

Caporale, G. M. and Pittis, N. (2001): Persistence in Macro Economic Time Series: Is it a Model Invariant Property?. Revista De Economia Del Rosario, 4:117-142. 
Chen, S., Min, W. \& Chen, R. (2013): Model Identification for Time Series with Dependent Innovation. Statistica Sinica, 23(2):873-899.

Christoffersen, P. (1998), Evaluating Interval Forecasts, International Economic Review, 39, 841-862.

Christoffersen, P. and Pelletier, D. (2004): Backtesting value-at-risk: A duration-based approach, Journal of Financial Econometrics, 2(1), 84-108.

Christoffersen, P., Hahn,J. and Inoue, A. (2001), Testing and Comparing Value-at-Risk Measures, Journal of Empirical Finance, 8, 325-342.

Christoffersen, P., Jacobs, K., Ornthanalai, C., Wang, Y., (2008). Option valuation with long- run and short-run volatility components. Journal of Financial Economics 90, 272-297.

Chu, J.; Chan, S.; Nadarajah, S. and Osterrieder, J.(2017): GARCH Modeling of Cryptocurrencies. Jounal of Risk and Financial Management, 10(17):1-15

Cooray, T. M. J. A.(2008): Applied Time series Analysis and Forecasting. New Delhi: $\quad$ Narosa Publising House.

Dhamija and Bhalla (2010) "Financial time series forecasting: comparison of neural networks and ARCH models", International Research Journal of Finance and Management, 49 (1), 159-172

Ding, Z, Granger, C. W. J. \& Engle, R. F. (1993) “A long memory property of stock market returns and a new model”, Journal of Empirical Finance, 1, 83-106.

Dobre I \& Alexandru AA 2008. Modelling Unemployment Rate using Box-Jenkins Procedure. Journal of Applied Quantitative Methods. 3(2): 156166.

Emenike, K. O. and Ani, W. U.(2014): Volatility of the Banking Sector Returns in Nigeria. Ruhuna Journal of Management and Finance, 1(1):73-82.

Emenogu, G. N. and Adenomon, M. O(2018); The Effect of High Positive Autocorrelation on the Performance of GARCH Family Models. Preprints. doi:10.20944/preprints201811.0381.v1

Emenogu, G. N. and Adenomon, M. O. 2018. The Effect of High Positive Autocorrelation on the Performance of GARCH Family Models. Preprints, doi:10.20944/preprints201811.0381.v1 
Emenogu, G. N.; Adenomon, M. O. and Nweze, N. O. 2018. On The Volatility of Daily Stock Returns of Total Petroleum Company of Nigeria: Evidence from GARCH Models, Value-at-Risk and Backtesting. Preprints, doi:10.20944/preprints201812.0043.v1

Enders, W., (2004). Applied Econometric Time Series. John Wiley, New York.

Engle, R. F. and Patton, A. J. (2001): What Good is Volatility Model?. Quantitative Finance, 1:237-245.

Engle, R., and Rangel, J., (2008). The spline-GARCH model for low-frequency volatility and its global macroeconomic causes. Review of Financial Studies 21, 1187-1222.

Enocksson, D. and Skoog, J. (2012) Evaluating VaR (Value-at-Risk): with the ARCH/GARCH class models via, European Union: Lambert Academic Publishing (LAP).

Francq, C. \& Zakoian, J-M. (2004): Maximum Likelihood Estimation of Pure GARCH and ARMA-GARCH Processes. Bernoulli, 10(4):605-637.

Ghalanos, A.(2018): Package 'rugarch'. R Team Cooperation.

Grek, A. (2014) "Forecasting accuracy for ARCH models and $\operatorname{GARCH}(1,1)$ family which model does best capture the volatility of the Swedish stock market"? Statistics Advance Level Theses 15hp; Örebro University.

Grigoletto, M. and Lisi, F.(2009): Looking for Skewness in Financial Time Series. The Econometrics Journal, 12(2):310-323.

Gujarati DN 2003. Basic Econometrics, $4^{\text {th }}$ edn. The McGraw-Hill Co, New Delhi, pp. 835848

Hall, P., and P., Yao, (2003). "Inference in ARCH and GARCH Models with Heavy-Tailed Errors", Econometrica, 71, 285-317.

Hansen, P. R. and Lunde, A.(2005): A Forecast Comparison of Volatility Models: Does Anything Beat a GARCH (1,1)? Journal of Applied Econometrics, 20:873-889.

Heracleous, M. S.(2003): Volatility Modeling Using the Student's t Distribution. Ph.D Thesis, Virginia Polytechnic Institute and State University, Blacksburg, Virginia.

Hsieh, K. C. \& Ritchken, P. (2005) “An Empirical Comparison of GARCH Option Pricing Models". Review of Derivatives Research, 8 (3): 129 -150. 
Jiang, W. (2012) "Using the GARCH model to analyse and predict the different stock markets" Master Thesis in Statistics, Department of Statistics, Uppsala University Sweden.

Kongcharoen, C. and Kruangpradit, T. 2013. Autoregressive Integrated Moving Average with Explanatory Variable (ARIMAX) Model for Thailand Export. A paper presented at the $33^{\text {rd }}$ International Symposium on Forecasting, South Korea, June 2013.

Kononovicius, A. \& Ruseckas, J. (2015) "Nonlinear GARCH Model and 1/f noise". ARXIV:1412.6244V2[Q-FIN.ST].

Koul, H. L. \& Ling, S. (2006): Fitting an Error Distribution in Some Heteroscedastic Time Series Models. The Annals of Statistics, 34(2):994-1012.

Kuhe, D. A. (2018): Modeling Volatility Persistence and Asymmetry with Exogenous Breaks in the Nigerian Stock Returns. CBN Journal of Applied Statistics, 9(1):167-196.

Lange, T.(2011): Tail Behavior and OLS Estimating in AR-GARCH Models. Statistica Sinica, 21(3):1191-1200.

Lanne, M. \& Saikkonen, P. (2005) "Nonlinear GARCH Models for Highly Persistent Volatility”. Econometrics Journal, 8 (2): 251-276.

Lawrance, A. J.(2013): Exploration Graphics for Financial Time Sewries Volatility. Journal of the Royal Statistical Society Series C (Applied Statistics), 62(5): 669-686.

Ling, S. \& McAleer, M. (2003): On Adaptive Estimation in Non Stationary ARMA Models with GARCH Errors. The Annals of Statistics, 31(2):642-674.

Magnus, F. J. and Fosu, A. E.(2006): Modelling and Forecasting Volatility of Returns on the Ghana Stock Exchange Using GARCH Models. Am. J. Appl. Sci. 3(10): 2042-2048.

Malecka, M. (2014) "GARCH Class Models Performance in Context of High Market Volatility”. ACTA Universitatis Lodziensis Folia Oeconmica, 3: 253 -266

Marra, S.(2015): Predicting Volatility. Investment Research. LAZARD Asset Management (LR26017), Australia.

Meitz, M. \& Saikkonen, P.(2011): Parameter Estimation in Non Linear AR-GARCH Models. Econometric Theory, 27(6):1236-1278.

Moshiri, S. \& Foroutan, F.(2006): Forecasting Non Linear Crude Oil Futures Prices. The Energy Journal, 27(4):81-95 
Nelson, D., (1991). Conditional heteroskedasticity in asset pricing: A new approach. Econometrica 59, 347-370.

Nieppola, O.(2009): Backtesting Value-at-Risk Models. M.Sc. Thesis, Helsinki School of Economics, Finland.

Panait, I. and Slavescu, E. O.(2012): Using GARCH-in-Mean Model to Investigate Volatility and Persistence at Different Frequencies for Bucharest Stock Exchange During 1997 -2012. Theoretical and Applied Economics, XIX(5):55-76.

Pedroni, P.(2001): The Econometric Modeling of Financial Time Series by Terence Mills. Journal of the American Statistical Association, 96(453): 345-346.

Peterson, B. G.; Carl, P.; Boudt, K.; Bennett, R.; Ulrich, J; Zivot, E.; Cornilly, D.; Hung, E.; Lestel, M.; Balkissoon, K. and Wuertz, D.(2018): Package 'PerformanceAnalytics'. R Team Cooperation

Rossi, E. (2004) "Lecture notes on GARCH models". University of Pavia, March.

Ruppert, D.(2011): Statistics and Data Analysis for Financial Engineering. New York: Springer Science + Business Media

Sahai, A. K. (2016): Volatility Modelling and Forecasting Efficiency of GARCH Models on Soy Oil Futures in India and the US. Journal of Energy Technology and Policy, 6(3):32-38.

Starica, C. (2003): Is GARCH $(1,1)$ as Good a Model as the Nobel Prize Accolades would Imply? www.math.chalmers.se/starica downloaded on 27-02-2019

The European Financial Review (2012): Guaranty Trust Bank Plc. World, Financial Industry, Special features, Emerging \& Frontier Markets, Banking \& Insurance, Industry Insights, October $2^{\text {nd }} 2012$. www.europeanfinancialreview.com/?p=1384 downloaded on 17-01 -2019 .

Tsay, R. S. (2005) Analysis of financial time series, 2nd Edition. New Jersey: John Wiley \& Sons.

Usman, U.; Auwal, H. M. and Abdulmuhyi, M. A.(2017): Fitting the Nigeria Stock Returns Using GARCH Models. Theoretical Economics Letters, 7:2159-2176.

Vosvrda, M.(2006): Empirical Analysis of Persistence and Dependence Patterns Among the Capital Markets. Prague Economic Papers, 3:231-242. 
Wilhelmsson, A. (2006): GARCH Forecasting Performance under Different Distribution Assumptions. Journal of Forecasting, 25:561-578.

Zhu, K. \& Ling, S.(2011): Global Self-Weighted and Local Quasi-Maximum Exponential Likelihood Estimators for ARMA-GARCH/IGARCH Models. The Annals of Statistics, 39(4): 2131-2163. 
Table 8: Estimates of ARMA(1,1)-eGARCH(1,1) with std

\begin{tabular}{|c|c|c|c|c|}
\hline \multicolumn{5}{|c|}{ 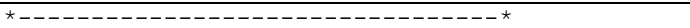 } \\
\hline \multicolumn{5}{|c|}{ * GARCH Model Fit * } \\
\hline \multicolumn{5}{|c|}{$\begin{array}{l}\text { *--- } \\
\text { Conditional Variance Dynamics }\end{array}$} \\
\hline \multicolumn{5}{|c|}{ 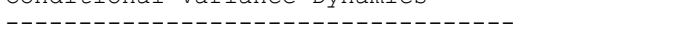 } \\
\hline \multicolumn{5}{|c|}{ GARCH Model $\quad: \operatorname{egARCH}(1,1)$} \\
\hline \multicolumn{2}{|c|}{ Mean Model } & \multicolumn{3}{|c|}{ : $\operatorname{ARFIMA}(1,0,1)$} \\
\hline \multicolumn{2}{|c|}{ Distribution } & \multicolumn{2}{|c|}{ : std } & \\
\hline \multicolumn{5}{|c|}{ Optimal Parameters } \\
\hline \multicolumn{5}{|c|}{------------------------------------- } \\
\hline & Estimate & Std. Error & t value & $\operatorname{Pr}($ \\
\hline $\operatorname{ar} 1$ & 0.090426 & 0.135239 & 0.66864 & \\
\hline ma1 & 0.015825 & 0.133828 & 0.11825 & \\
\hline omega & -0.638445 & 0.020334 & -31.39719 & \\
\hline alphal & 0.192669 & 0.052765 & 3.65144 & \\
\hline betal & 0.882287 & 0.001346 & 655.31371 & \\
\hline gamma 1 & 1.831176 & 019753 & 92.70 & \\
\hline shape & 2.100000 & 0.009205 & 228.12482 & \\
\hline \multicolumn{5}{|c|}{ Robust Standard Errors: } \\
\hline & Estimate & Std. Error & t value & $\operatorname{Pr}(>|t|)$ \\
\hline $\operatorname{ar} 1$ & 0.090426 & 0.143390 & 0.63063 & 0.52828 \\
\hline mal & 0.015825 & 0.149513 & 0.10584 & 0.91571 \\
\hline omega & -0.638445 & 0.081165 & -7.86604 & 0.00000 \\
\hline alphal & 0.192669 & 0.171535 & 1.12321 & 0.26135 \\
\hline betal & 0.882287 & 0.002625 & 336.12545 & 0.00000 \\
\hline gamma 1 & 1.831176 & 0.357599 & 5.12075 & \\
\hline shape & 2.100000 & 0.049998 & 42.00152 & 0.00000 \\
\hline
\end{tabular}

LogLikelihood : 9737.114

$\begin{array}{ll}\text { Information } & \text { Criteria } \\ \text { Akaike } & -4.8457 \\ \text { Bayes } & -4.8347 \\ \text { Shibata } & -4.8457 \\ \text { Hannan-Quinn } & -4.8418\end{array}$

Weighted Ljung-Box Test on Standardized Residuals

$\begin{array}{lrr} & \text { statistic } & p-v a l u e \\ \operatorname{Lag}[1] & 0.06633 & 0.7968 \\ \operatorname{Lag}\left[2^{*}(p+q)+(p+q)-1\right][5] & 0.08466 & 1.0000 \\ \operatorname{Lag}[4 *(p+q)+(p+q)-1][9] & 0.23001 & 1.0000 \\ \text { d.o.f }=2 & & \\ \text { H0 : No serial correlation } & \end{array}$

Weighted Ljung-Box Test on Standardized Squared Residuals

$\begin{array}{llr} & \text { statistic } & p-v a l u e \\ \operatorname{Lag}[1] & 0.0004513 & 0.9831 \\ \operatorname{Lag}[2 *(p+q)+(p+q)-1][5] & 0.0013750 & 1.0000 \\ \operatorname{Lag}[4 *(p+q)+(p+q)-1][9] & 0.0022635 & 1.0000 \\ \text { d.o.f } f=2 & & \end{array}$

Weighted ARCH LM Tests

Statistic Shape Scale P-Value $\begin{array}{llllll}A R C H & \operatorname{Lag}[3] & 0.0004649 & 0.500 & 2.000 & 0.9828\end{array}$ ARCH Lag[5] $0.0011178 \quad 1.440 \quad 1.667 \quad 1.0000$ ARCH Lag[7] $0.0016103 \quad 2.315 \quad 1.543 \quad 1.0000$

Nyblom stability test

Joint Statistic: 10.9421

Individual Statistics:

ar1 1.2313

mal 1.2554

omega 2.7533

alpha1 0.8187

betal 2.1565

gamma 10.6688

shape 0.2326

\section{Appendix A}

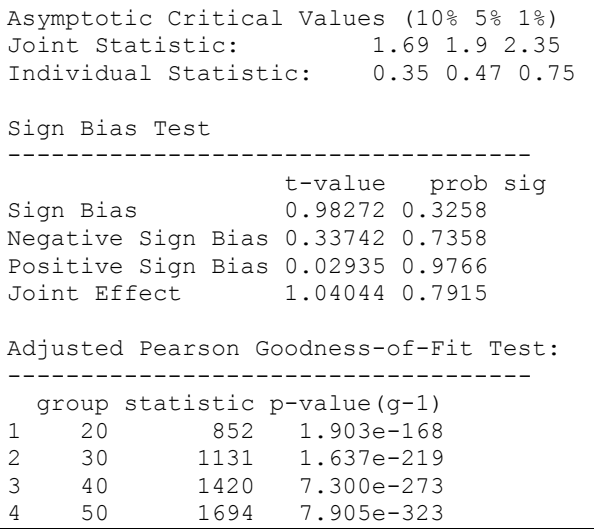

Table 9: Estimates of ARMA(1,1)-eGARCH(1,1) with sstd

\begin{tabular}{|c|c|c|c|c|}
\hline \multicolumn{5}{|c|}{ * $\quad$ GARCH Model Fit } \\
\hline \multicolumn{5}{|c|}{ Conditional Variance Dynamics } \\
\hline \multirow{3}{*}{\multicolumn{2}{|c|}{$\begin{array}{l}\text { GARCH Model } \\
\text { Mean Model } \\
\text { Distribution }\end{array}$}} & \multicolumn{3}{|c|}{ : $\operatorname{eGARCH}(1,1)$} \\
\hline & & \multicolumn{3}{|c|}{$: \operatorname{ARFIMA}(1,0,1)$} \\
\hline & & : sstd & & \\
\hline \multicolumn{5}{|c|}{ Optimal Parameters } \\
\hline \multicolumn{5}{|c|}{------------------------------------ } \\
\hline & Estimate & Std. Error & t value & $\operatorname{Pr}(>|t|)$ \\
\hline $\operatorname{ar} 1$ & 0.090252 & 0.059767 & 1.510 & \\
\hline ma1 & 0.013462 & 0.058206 & 0.23129 & \\
\hline omega & -0.355651 & 0.021578 & -16.48217 & \\
\hline alphal & 0.617628 & 0.165270 & 3.73708 & 0186 \\
\hline beta1 & 0.885358 & 0.004641 & 190.76635 & 000 \\
\hline gamma1 & 5.501824 & 0.189529 & 29.02895 & 0.000000 \\
\hline skew & 1.000633 & 0.009679 & 103.38505 & 0.000000 \\
\hline shape & 2.010000 & 0.000613 & 3278.52434 & 0 . \\
\hline \multicolumn{5}{|c|}{ Robust Standard Errors: } \\
\hline & Estimate & Std. Error & t value & $\operatorname{Pr}(>|t|)$ \\
\hline $\operatorname{ar} 1$ & 0.090252 & 0.038433 & 2.34831 & 0.018859 \\
\hline mal & 0.013462 & 0.025677 & 0.52431 & 0.600066 \\
\hline omega & -0.355651 & 0.091076 & -3.90499 & 0.000094 \\
\hline alpha1 & 0.617628 & 0.518456 & 1.19128 & 0.233542 \\
\hline beta1 & 0.885358 & 0.026923 & 32.88446 & 0.000000 \\
\hline gamma 1 & 5.501824 & 0.598899 & 9.18656 & 0.000000 \\
\hline skew & 1.000633 & 0.007893 & 126.77568 & 0.000000 \\
\hline shape & 2.010000 & 0.001837 & 1094.18647 & 0.000000 \\
\hline
\end{tabular}

LogLikelihood : 9742.685

Information Criteria

$\begin{array}{ll}\text { Akaike } & -4.8480 \\ \text { Bayes } & -4.8354 \\ \text { Shibata } & -4.8480 \\ \text { Hannan-0uinn } & -4.8435\end{array}$

Hannan-Quinn -4.8435

Weighted Ljung-Box Test on Standardized Residuals

Lag [1] $\begin{array}{rr}\text { Statistic } & p \text {-value } \\ 0.07050 & 0.7906\end{array}$

$\operatorname{Lag}\left[2^{*}(p+q)+(p+q)-1\right][5] \quad 0.08858 \quad 1.0000$

$\operatorname{Lag}\left[4^{*}(p+q)+(p+q)-1\right][9] \quad 0.23150 \quad 1.0000$

d.o.f $=2$

HO : No serial correlation

Weighted Ljung-Box Test on Standardized Squared Residuals 


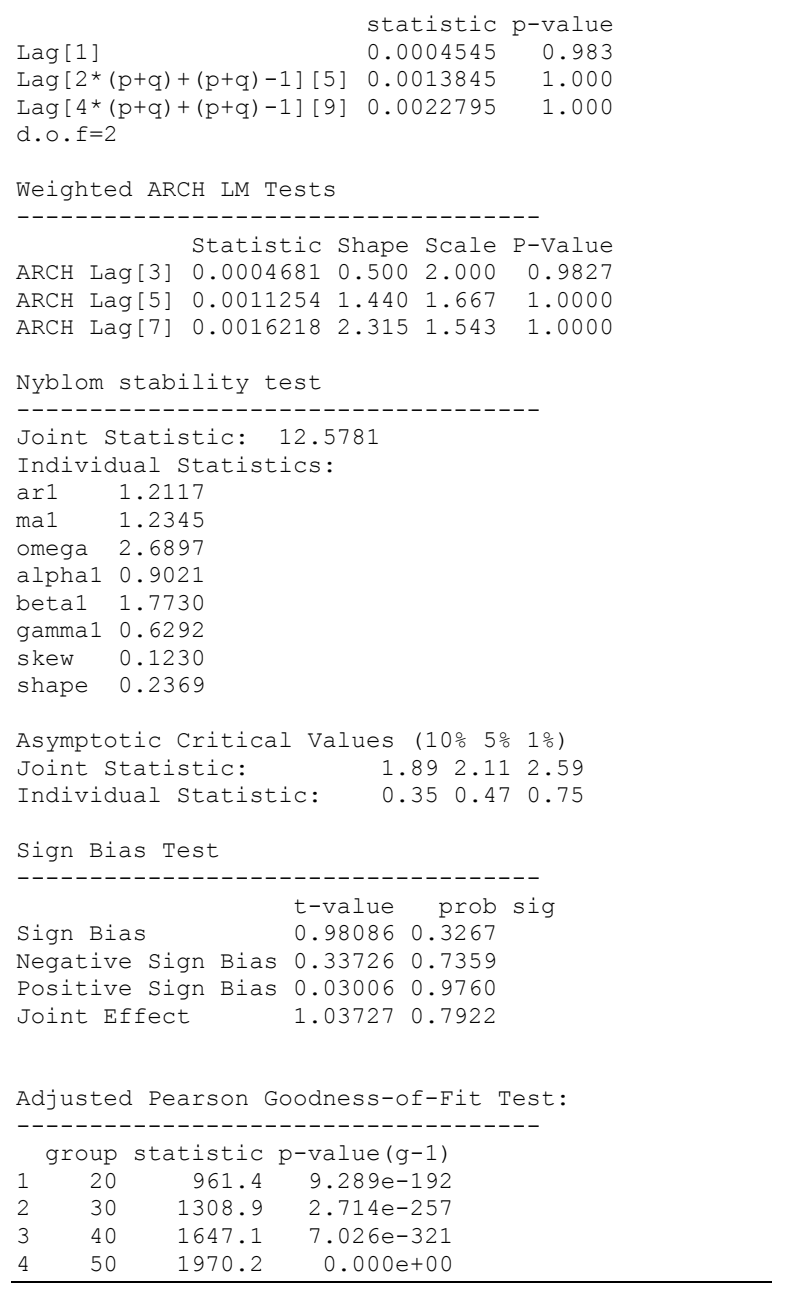

Table 10: Estimates of ARMA(1,1)-TGARCH(1,1) with std

\begin{tabular}{|c|c|c|c|c|}
\hline \multicolumn{5}{|c|}{ 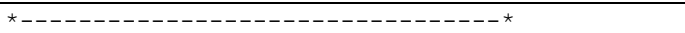 } \\
\hline \multicolumn{5}{|c|}{ * GARCH Model Fit * } \\
\hline \multirow{2}{*}{\multicolumn{5}{|c|}{ Conditional Variance Dynamics }} \\
\hline & & & ------- & \\
\hline \multicolumn{5}{|c|}{ GARCH Model : $\operatorname{fGARCH}(1,1)$} \\
\hline \multicolumn{5}{|c|}{ fGARCH Sub-Model : TGARCH } \\
\hline \multicolumn{5}{|c|}{ Mean Model : $\operatorname{ARFIMA}(1,0,1)$} \\
\hline \multicolumn{5}{|c|}{ Distribution : std } \\
\hline \multicolumn{5}{|c|}{ Optimal Parameters } \\
\hline \multicolumn{5}{|c|}{ 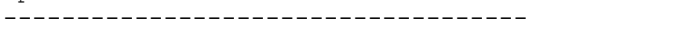 } \\
\hline & Estimate & Std. Error & t value $\mathrm{P}$ & $\operatorname{Pr}(>|t|)$ \\
\hline $\operatorname{ar} 1$ & 0.260521 & 0.018296 & 14.23931 & 0.00000 \\
\hline ma1 & -0.111401 & 0.018403 & -6.05358 & 0.00000 \\
\hline omega & 0.000000 & 0.000000 & 0.18102 & 0.85635 \\
\hline alpha1 & 0.695885 & 0.015610 & 44.58006 & 0.00000 \\
\hline betal & 0.499255 & 0.008670 & 57.58337 & 0.00000 \\
\hline eta11 & -0.005608 & 0.021908 & -0.25598 & 0.79797 \\
\hline shape & 3.117434 & 0.057788 & 53.94586 & 0.00000 \\
\hline \multicolumn{5}{|c|}{ Robust Standard Errors: } \\
\hline & Estimate & Std. Error & t value & $\operatorname{Pr}(>|t|)$ \\
\hline $\operatorname{ar} 1$ & 0.260521 & 0.327748 & 0.794880 & 0.426683 \\
\hline ma1 & -0.111401 & 0.423033 & -0.263339 & 0.792289 \\
\hline omega & 0.000000 & 0.000065 & 0.000551 & 0.999560 \\
\hline alphal & 0.695885 & 2.953122 & 0.235644 & 0.813709 \\
\hline betal & 0.499255 & 2.233770 & 0.223503 & 0.823144 \\
\hline
\end{tabular}

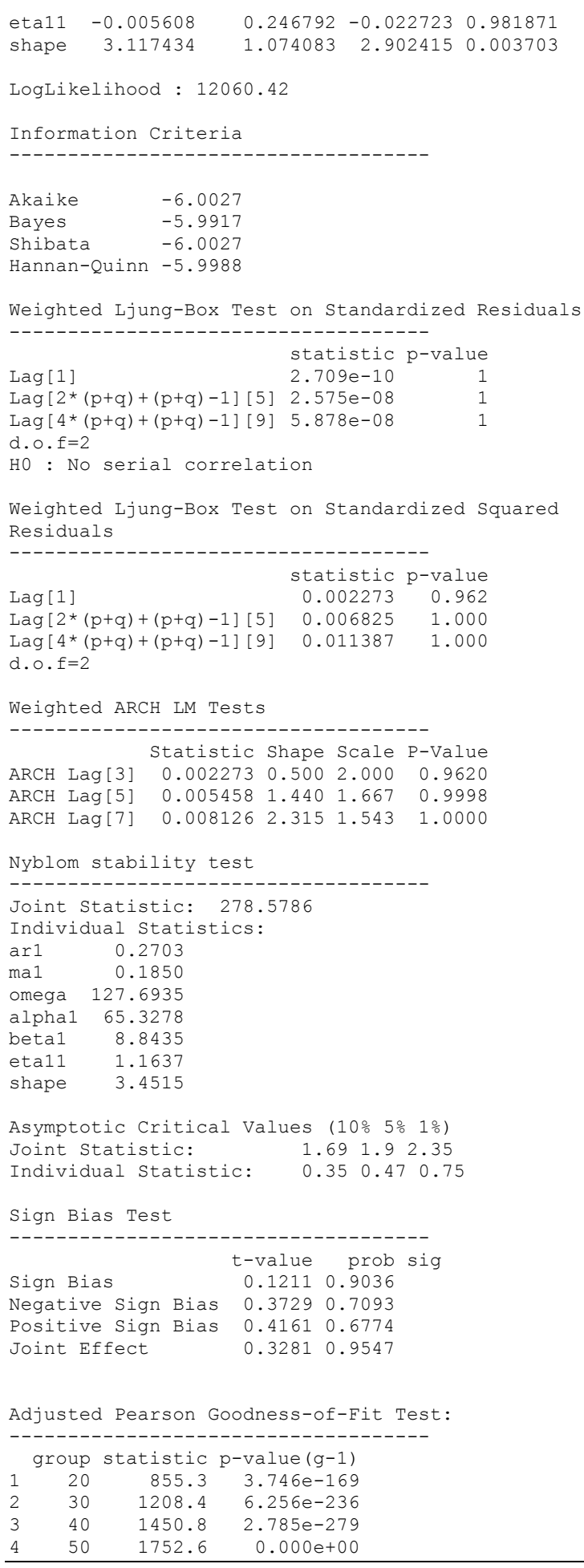

\begin{tabular}{rrrr} 
& group & statistic & p-value $(g-1)$ \\
2 & 20 & 855.3 & $3.746 e-169$ \\
3 & 30 & 1208.4 & $6.256 e-236$ \\
4 & 40 & 1450.8 & $2.785 e-279$ \\
\hline
\end{tabular}


Table 11: Estimates of ARMA(1,1)-TGARCH(1,1) with sstd

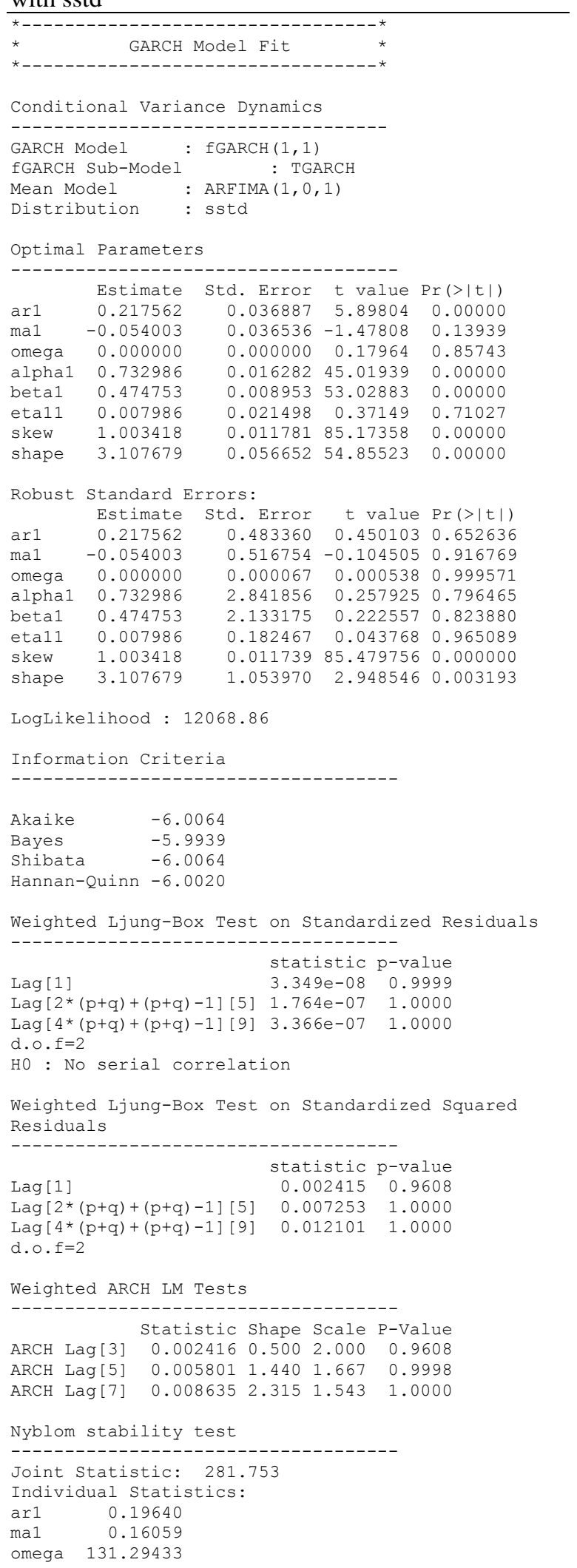

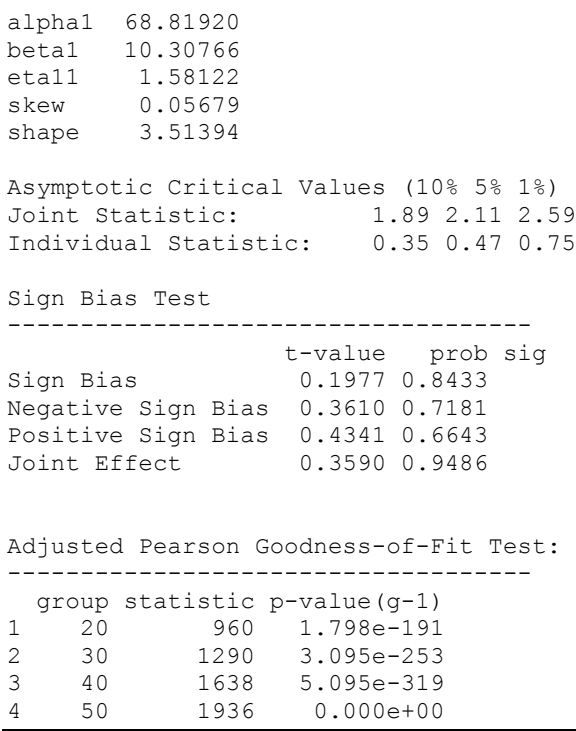

Table 12: Estimates of ARMA(1,1)-NAGARCH(1,1) with std

\begin{tabular}{|c|c|c|c|c|}
\hline \multicolumn{5}{|c|}{ 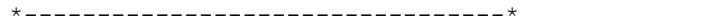 } \\
\hline \multicolumn{5}{|c|}{ * GARCH Model Fit * } \\
\hline \multicolumn{5}{|c|}{ 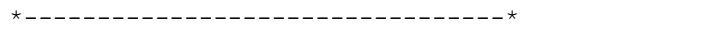 } \\
\hline \multicolumn{5}{|c|}{ Conditional Variance Dynamics } \\
\hline \multicolumn{5}{|c|}{ 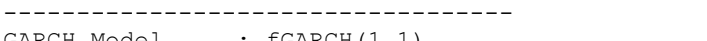 } \\
\hline \multicolumn{5}{|c|}{$\begin{array}{l}\text { GARCH Model } \\
\text { fGARCH Sub-Model } \begin{array}{c}\text { fGARCH }(1,1) \\
: \text { NAGARCH }\end{array}\end{array}$} \\
\hline \multicolumn{5}{|c|}{ Mean Model $\quad: \operatorname{ARFIMA}(1,0,1)$} \\
\hline \multicolumn{5}{|c|}{ Distribution $\quad$ : std } \\
\hline \multicolumn{5}{|c|}{ Optimal Parameters } \\
\hline \multicolumn{5}{|c|}{ - } \\
\hline & Estimate & Std. Error & t value & $\operatorname{Pr}(>|t|)$ \\
\hline $\operatorname{ar} 1$ & 0.202254 & 0.161208 & 1.25462 & 0.20962 \\
\hline ma1 & -0.136256 & 0.166396 & -0.81887 & 0.41286 \\
\hline omega & 0.000000 & 0.000000 & 0.11911 & 0.90519 \\
\hline alphal & 0.348048 & 0.012211 & 28.50355 & 0.00000 \\
\hline betal & 0.643824 & 0.009687 & 66.46287 & 0.00000 \\
\hline eta21 & 0.043594 & 0.089369 & 0.48779 & 0.62570 \\
\hline shape & 3.715735 & 0.102281 & 36.32859 & 0.00000 \\
\hline \multicolumn{5}{|c|}{ Robust Standard Errors: } \\
\hline & Estimate & Std. Error & t value & $\operatorname{Pr}(>|t|)$ \\
\hline $\operatorname{ar} 1$ & 0.202254 & 0.414763 & 0.48764 & 0.625807 \\
\hline $\operatorname{ma1}$ & -0.136256 & 0.515821 & -0.26415 & 0.791661 \\
\hline omega & 0.000000 & 0.000073 & 0.00049 & 0.999609 \\
\hline alpha1 & 0.348048 & 1.863233 & 0.18680 & 0.851819 \\
\hline beta1 & 0.643824 & 1.833290 & 0.35119 & 0.725450 \\
\hline eta21 & 0.043594 & 0.332024 & 0.13130 & 0.895540 \\
\hline shape & 3.715735 & 1.523823 & 2.43843 & 0.014751 \\
\hline \multicolumn{5}{|c|}{ LogLikelihood : 10162.54} \\
\hline \multicolumn{5}{|c|}{ Information Criteria } \\
\hline \multicolumn{5}{|c|}{ Akaike $\quad-5.0575$} \\
\hline \multicolumn{5}{|c|}{ Bayes $\quad-5.0466$} \\
\hline \multicolumn{5}{|c|}{ Shibata $\quad-5.0575$} \\
\hline \multicolumn{5}{|c|}{ Hannan-Quinn -5.0536} \\
\hline \multicolumn{5}{|c|}{ Weighted Ljung-Box Test on Standardized Residuals } \\
\hline \multicolumn{5}{|c|}{ statistic $p$-value } \\
\hline \multicolumn{5}{|c|}{$\begin{array}{lll}\operatorname{Lag}[1] & 0.03067 & 0.861\end{array}$} \\
\hline \multicolumn{3}{|c|}{$\begin{array}{l}\operatorname{Lag}[2 *(p+q)+(p+q)-1][5] \\
\operatorname{Lag}[4 *(p+q)+(p+q)-1][9]\end{array}$} & 3263 & .000 \\
\hline \multicolumn{5}{|c|}{$\begin{array}{l}\operatorname{Lag}\left[4^{\star}(p+q)+(p+q)-1\right][9] \\
d . \circ . f=2\end{array}$} \\
\hline
\end{tabular}


HO : No serial correlation

Weighted Ljung-Box Test on Standardized Squared Residuals

$\begin{array}{lrr} & \text { statistic } & p \text {-value } \\ \operatorname{Lag}[1] & 0.001840 & 0.9658 \\ \operatorname{Lag}[2 *(p+q)+(p+q)-1][5] & 0.006133 & 1.0000 \\ \operatorname{Lag}[4 *(p+q)+(p+q)-1][9] & 0.010398 & 1.0000 \\ \text { d.o.f }=2 & & \end{array}$

d. o. $f=2$

Weighted ARCH LM Tests

Statistic Shape Scale P-Value

$\begin{array}{llllll}A R C H & \operatorname{Lag}[3] & 0.002144 & 0.500 & 2.000 & 0.9631\end{array}$

$\begin{array}{llllll}\mathrm{ARCH} & \operatorname{Lag}[5] & 0.005173 & 1.440 & 1.667 & 0.9998\end{array}$

$\begin{array}{llllll}A R C H & \operatorname{Lag}[7] & 0.007636 & 2.315 & 1.543 & 1.0000\end{array}$

Nyblom stability test

Joint Statistic: 230.4385

Individual Statistics:

$\begin{array}{ll}\operatorname{ar} 1 & 0.3625\end{array}$

mal 0.3992

omega 101.8067

alpha1 51.9765

beta1 7.8753

eta21 1.3598

shape $\quad 4.1795$

Asymptotic Critical Values (10\% 5\% 1\%)

Joint Statistic: $\quad \begin{array}{lllll}1.69 & 1.92 .35\end{array}$

Individual Statistic: $\quad 0.350 .470 .75$

Sign Bias Test

$\begin{array}{lrl} & & \\ \text { Sign Bias } & \text { t-value } & \text { prob sig } \\ \text { Negative Sign Bias } & 0.4702 & 0.3628 \\ \text { Positive Sign Bias } & 0.1879 & 0.6333 \\ \text { Joint Effect } & 1.0520 & 0.7887\end{array}$

Adjusted Pearson Goodness-of-Fit Test:

\begin{tabular}{rrrr} 
& group statistic & p-value $(g-1)$ \\
2 & 20 & 1161 & $2.433 e-234$ \\
3 & 30 & 1491 & $4.173 e-296$ \\
4 & 50 & 1744 & $0.000 e+00$ \\
\hline
\end{tabular}

Table 13: Estimates of ARMA(1,1)-NAGARCH(1,1) with sstd

\begin{tabular}{|c|c|c|c|c|}
\hline \multicolumn{5}{|c|}{ - - - } \\
\hline \multicolumn{5}{|c|}{ * GARCH Model Fit * } \\
\hline \multirow{2}{*}{\multicolumn{5}{|c|}{ Conditional Variance Dynamics }} \\
\hline & & & & \\
\hline \multicolumn{5}{|c|}{ GARCH Model : fGARCH $(1,1)$} \\
\hline \multicolumn{5}{|c|}{ fGARCH Sub-Model : NAGARCH } \\
\hline \multicolumn{5}{|c|}{ Mean Model : $\operatorname{ARFIMA}(1,0,1)$} \\
\hline \multicolumn{5}{|c|}{ Distribution : sstd } \\
\hline \multicolumn{5}{|c|}{ Optimal Parameters } \\
\hline \multicolumn{5}{|c|}{ 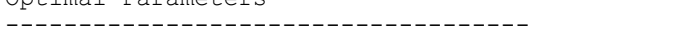 } \\
\hline & Estimate & Std. Error & t value & $\operatorname{Pr}(>|t|)$ \\
\hline $\operatorname{ar} 1$ & 0.21159 & 0.166371 & 1.27179 & 0.20345 \\
\hline ma1 & -0.14934 & 0.172436 & -0.86605 & 0.38646 \\
\hline omega & 0.00000 & 0.000000 & 0.11943 & 0.90494 \\
\hline alpha1 & 0.34296 & 0.011964 & 28.66595 & 0.00000 \\
\hline betal & 0.64255 & 0.009524 & 67.46838 & 0.00000 \\
\hline eta21 & 0.01319 & 0.112685 & 0.11705 & 0.90682 \\
\hline skew & 1.00799 & 0.012574 & 80.16590 & 0.00000 \\
\hline shape & 3.73914 & 0.102750 & 36.39075 & 0.00000 \\
\hline
\end{tabular}

Robust Standard Errors:

$\begin{array}{lrrrr} & \text { Estimate } & \text { Std. Error } & t \text { value } \operatorname{Pr}(>|t|) \\ \text { ar1 } & 0.21159 & 0.251290 & 0.842011 & 0.399782 \\ \text { ma1 } & -0.14934 & 0.358117 & -0.417011 & 0.676671 \\ \text { omega } & 0.00000 & 0.000073 & 0.000492 & 0.999607 \\ \text { alpha1 } & 0.34296 & 1.829657 & 0.187445 & 0.851312 \\ \text { beta1 } & 0.64255 & 1.838481 & 0.349501 & 0.726713 \\ \text { eta21 } & 0.01319 & 1.457099 & 0.009052 & 0.992778 \\ \text { skew } & 1.00799 & 0.041364 & 24.368965 & 0.000000 \\ \text { shape } & 3.73914 & 1.625545 & 2.300239 & 0.021435\end{array}$

LogLikelihood : 10152.18

Information Criteria

Akaike $\quad-5.0519$

Bayes $\quad-5.0393$

Shibata $\quad-5.0519$

Hannan-Quinn -5.0474

Weighted Ljung-Box Test on Standardized Residuals

$\begin{array}{lrr} & \text { statistic } & p-v a l u e \\ \operatorname{Lag}[1] & 0.03295 & 0.856 \\ \operatorname{Lag}\left[2^{*}(p+q)+(p+q)-1\right][5] & 0.03493 & 1.000 \\ \operatorname{Lag}[4 *(p+q)+(p+q)-1][9] & 0.05748 & 1.000 \\ \text { d } f=2 & & \end{array}$

d. $\circ . f=2$

HO : No serial correlation

Weighted Ljung-Box Test on Standardized squared Residuals

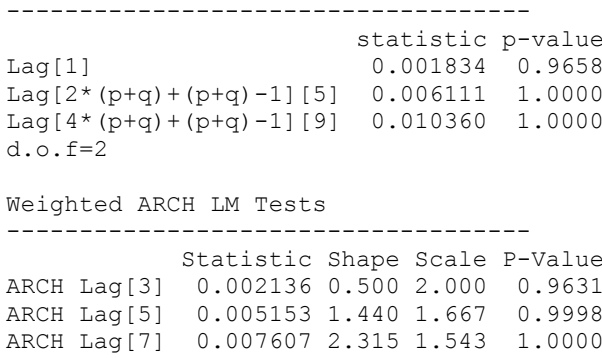

Nyblom stability test

Joint Statistic: 232.8581

Individual Statistics:

ar1 0.37484

ma1 0.41837

omega 101.51981

alpha1 55.11488

beta1 8.72212

eta21 0.65460

skew $\quad 0.07016$

shape $\quad 4.15469$

Asymptotic Critical Values (10\% 5\% 1\%)

Joint Statistic: 1.892 .112 .59

Individual Statistic: $\quad \begin{array}{lllll}0.35 & 0.47 & 0.75\end{array}$

Sign Bias Test

$\begin{array}{lrr} & & \\ \text { Sign Bias } & 0.9112 & 0.3622 \\ \text { Negative Sign Bias } & 0.4772 & 0.6333 \\ \text { Positive Sign Bias } & 0.1882 & 0.8508 \\ \text { Joint Effect } & 1.0544 & 0.7881\end{array}$

Adjusted Pearson Goodness-of-Fit Test:

$\begin{array}{rrrr} & \text { group statistic } & \text { p-value }(g-1) \\ 1 & 20 & 1255 & 1.619 e-254 \\ 2 & 30 & 1659 & 0.000 e+00 \\ 4 & 40 & 1917 & 0.000 e+00 \\ 4 & 50 & 2194 & 0.000 e+00\end{array}$


Table 14: Estimates of ARMA(1,1)-AVGARCH(1,1) with std

\begin{tabular}{|c|c|c|c|c|}
\hline \multicolumn{5}{|c|}{ * GARCH Model Fit * } \\
\hline \multicolumn{5}{|c|}{ 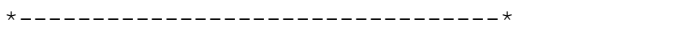 } \\
\hline \multicolumn{5}{|c|}{ Conditional Variance Dynamics } \\
\hline \multicolumn{5}{|c|}{ GARCH Model : fGARCH $(1,1)$} \\
\hline \multicolumn{5}{|c|}{ fGARCH Sub-Model : AVGARCH } \\
\hline \multicolumn{5}{|c|}{ Mean Model : $\operatorname{ARFIMA}(1,0,1)$} \\
\hline \multicolumn{5}{|c|}{ Distribution : std } \\
\hline \multicolumn{5}{|c|}{ Optimal Parameters } \\
\hline \multicolumn{5}{|c|}{---------------------------------------- } \\
\hline & Estimate & Std. Error & t value & $\operatorname{Pr}(>|t|)$ \\
\hline $\operatorname{ar} 1$ & 0.216232 & 0.020440 & 10.57901 & 0 . \\
\hline ma1 & -0.091422 & 0.022696 & -4.02803 & 0.0 \\
\hline omega & 0.000000 & 0.000000 & 0.17852 & 0.8 \\
\hline alpha1 & 0.722956 & 0.015914 & 45.42756 & 0.000000 \\
\hline beta1 & 0.476527 & 0.008804 & 54.12823 & 0.000000 \\
\hline eta11 & -0.026953 & 0.021307 & -1.26499 & 0.205876 \\
\hline eta21 & 0.000193 & 0.001045 & 0.18441 & 0.853692 \\
\hline shape & 3.141671 & 0.058494 & 53.70923 & 0.000000 \\
\hline \multicolumn{5}{|c|}{ Robust Standard Errors: } \\
\hline & Estimate & Std. Error & t value & $e \operatorname{Pr}(>|t|)$ \\
\hline $\operatorname{arc}$ & 0.216232 & 0.228626 & 0.945789 & 0.344256 \\
\hline $\operatorname{ma1}$ & -0.091422 & 0.543642 & -0.168165 & 0.866453 \\
\hline omega & 0.000000 & 0.000068 & 0.000531 & 10.999576 \\
\hline alpha1 & 0.722956 & 2.763034 & 0.261653 & 0.793589 \\
\hline betal & 0.476527 & 2.080736 & 0.229018 & 0.818855 \\
\hline eta11 & -0.026953 & 0.287347 & -0.093800 & 0.925268 \\
\hline eta21 & 0.000193 & 0.002573 & 0.074930 & 0.940270 \\
\hline shape & 3.141671 & 1.430933 & 2.195539 & 90.028125 \\
\hline
\end{tabular}

LogLikelihood : 12053.98

Information Criteria

$\begin{array}{ll}\text { Akaike } & -5.9990 \\ \text { Bayes } & -5.9864 \\ \text { Shibata } & -5.9990 \\ \text { Hannan-Quinn } & -5.9945\end{array}$

Hannan-Quinn -5.9945

Weighted Ljung-Box Test on Standardized Residuals

$\begin{array}{lll} & \text { statistic } & p-v a l u e \\ \operatorname{Lag}[1] & 3.257 e-08 & 0.9999 \\ \operatorname{Lag}\left[2^{*}(p+q)+(p+q)-1\right][5] & 1.757 e-07 & 1.0000 \\ \operatorname{Lag}[4 *(p+q)+(p+q)-1][9] & 3.368 e-07 & 1.0000 \\ \text { d.o.f=2 } & \\ \text { HO : No serial correlation } & \end{array}$

Weighted Ljung-Box Test on Standardized Squared Residuals

$\begin{array}{lrr} & \text { Statistic } & p \text {-value } \\ \operatorname{Lag}[1] & 0.002416 & 0.9608 \\ \operatorname{Lag}\left[2^{*}(p+q)+(p+q)-1\right][5] & 0.007254 & 1.0000 \\ \operatorname{Lag}[4 *(p+q)+(p+q)-1][9] & 0.012102 & 1.0000 \\ \text { d.o.f }=2 & & \end{array}$

$\mathrm{d} . \circ \cdot \mathrm{f}=2$

Weighted ARCH LM Tests

Statistic Shape Scale P-Value

$\begin{array}{llllll}A R C H & \operatorname{Lag}[3] & 0.002416 & 0.500 & 2.000 & 0.9608\end{array}$

$\begin{array}{llllll}\mathrm{ARCH} & \operatorname{Lag}[5] & 0.005801 & 1.440 & 1.667 & 0.9998\end{array}$

$\begin{array}{llllll}A R C H & \operatorname{Lag}[7] & 0.008636 & 2.315 & 1.543 & 1.0000\end{array}$

Nyblom stability test

Joint Statistic: 281.1214

Individual Statistics:

$\operatorname{arl} \quad 0.2856$

ma1 0.4489

$\begin{array}{lr}\text { omega } & 130.6555 \\ \text { alpha1 } & 69.7212 \\ \text { beta1 } & 11.1180 \\ \text { eta11 } & 0.5819 \\ \text { eta21 } & 0.3538 \\ \text { shape } & 3.9707\end{array}$

Asymptotic Critical Values (10\% 5\% 1)

Joint Statistic: $\quad \begin{array}{lllll}1.89 & 2.11 & 2.59\end{array}$

Individual Statistic: $\quad 0.350 .470 .75$

Sign Bias Test

$\begin{array}{lrr} & \text { t-value } & \text { prob sig } \\ \text { Sign Bias } & 0.1974 & 0.8435 \\ \text { Negative Sign Bias } & 0.3617 & 0.7176 \\ \text { Positive Sign Bias } & 0.4444 & 0.6568 \\ \text { Joint Effect } & 0.3668 & 0.9470\end{array}$

Adjusted Pearson Goodness-of-Fit Test:

$\begin{array}{rrrr} & \text { group } & \text { statistic } & \text { p-value }(\mathrm{g}-1) \\ 1 & 20 & 961 & 1.130 \mathrm{e}-191 \\ 2 & 30 & 1315 & 1.142 \mathrm{e}-258 \\ 3 & 40 & 1572 & 5.360 \mathrm{e}-305 \\ 4 & 50 & 1898 & 0.000 \mathrm{e}+00\end{array}$

Table 15: Estimates of ARMA(1,1)-AVGARCH(1,1) with sstd

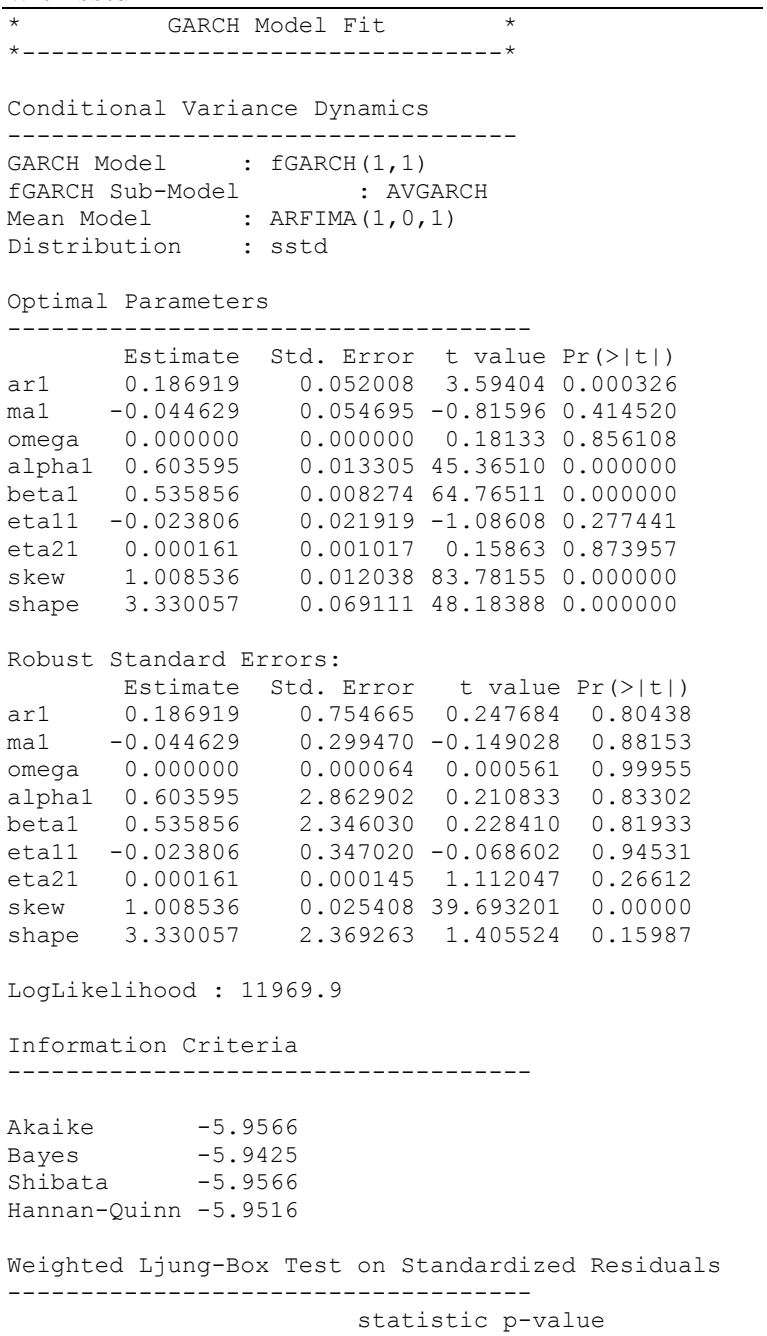




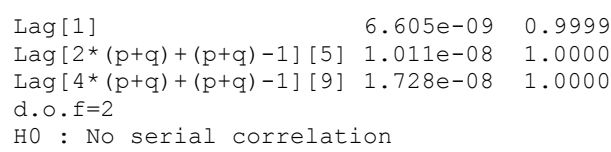

Asymptotic Critical Values (10\% 5\% $1 \%$ ) Joint Statistic: $\quad 2.12 .322 .82$ Individual Statistic: $\quad \begin{array}{lll}0.35 & 0.47 & 0.75\end{array}$

Sign Bias Test

t-value prob sig

Sign Bias

$\begin{array}{lll}0.04364 & 0.9652\end{array}$

Negative Sign Bias 0.388160 .6979

Positive Sign Bias $0.36206 \quad 0.7173$

Joint Effect $\quad 0.28283 \quad 0.9632$

Adjusted Pearson Goodness-of-Fit Test:

\begin{tabular}{rrrr} 
& group & statistic & p-value $(\mathrm{g}-1)$ \\
1 & 20 & 1035 & $1.779 \mathrm{e}-207$ \\
2 & 30 & 1371 & $2.107 \mathrm{e}-270$ \\
3 & 40 & 1672 & $0.000 \mathrm{e}+00$ \\
4 & 50 & 2011 & $0.000 \mathrm{e}+00$ \\
\hline
\end{tabular}

Table 16: Estimates of ARMA(1,1)-eGARCH(2,2) with std

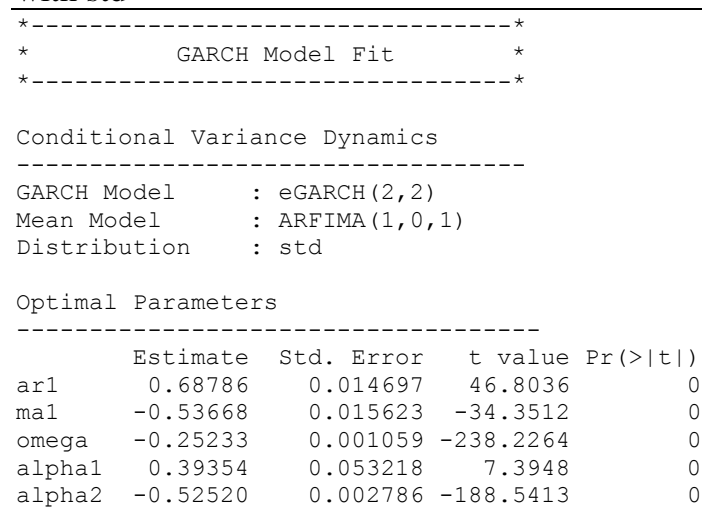

\begin{tabular}{|c|c|c|c|c|}
\hline beta1 & 0.73683 & 0.000159 & 4631.2482 & \\
\hline & 0.23768 & 0.000247 & 963.5852 & \\
\hline nal & 3.69469 & 0.006275 & 588.8134 & \\
\hline amma 2 & 0.44676 & 0.002619 & 170.5717 & \\
\hline hape & 2.10000 & 0.000751 & 2797.4873 & \\
\hline bust & \multicolumn{4}{|c|}{ Standard Errors: } \\
\hline & Estimate & std. Error & t value & $\operatorname{Pr}(>|t|)$ \\
\hline & 0.68786 & 0.048823 & 14.089 & 0.00000 \\
\hline & -0.53668 & 0.040005 & -13.415 & 0.00000 \\
\hline nega & -0.25233 & 0.004415 & -57.147 & 0.00000 \\
\hline Lhal & 0.39354 & 0.241286 & 1.631 & 0.10289 \\
\hline pha2 & -0.52520 & 0.003159 & -166.235 & 0.00000 \\
\hline ta1 & 0.73683 & 0.002479 & 297.207 & 0.00000 \\
\hline ta2 & 0.23768 & 0.002884 & 82.418 & 0.0000 \\
\hline nma 1 & 3.69469 & 0.073306 & 50.401 & 0.00000 \\
\hline nma2 & 0.44676 & 0.004863 & 91.866 & 0.00000 \\
\hline ape & 2.10000 & 0.003104 & 676.648 & 0.0000 \\
\hline
\end{tabular}

LogLikelihood : 10432.39

Information Criteria

$\begin{array}{ll}\text { Akaike } & -5.1904 \\ \text { Bayes } & -5.1748 \\ \text { Shibata } & -5.1904 \\ \text { Hannan-Quinn } & -5.1849\end{array}$

Weighted Ljung-Box Test on Standardized Residuals

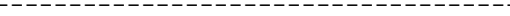

statistic p-value

$\operatorname{Lag}[2 *(p+q)+(p+q)-1][5] \quad 0.0019914 \quad 1.0000$ $\operatorname{Lag}[4 *(p+q)+(p+q)-1][9] \quad 0.0033223 \quad 1.0000$ d. $\circ . \mathrm{f}=2$

$\mathrm{HO}$ : No serial correlation

Weighted Ljung-Box Test on Standardized Squared Residuals

$\begin{array}{lrr} & \text { Statistic } & p \text {-value } \\ \operatorname{Lag}[1] & 0.0003884 & 0.9843 \\ \operatorname{Lag}[2 *(p+q)+(p+q)-1][11] & 0.0023365 & 1.0000 \\ \operatorname{Lag}[4 *(p+q)+(p+q)-1][19] & 0.0039019 & 1.0000 \\ \text { d. } 0 . f=4 & & \end{array}$

Weighted ARCH LM Tests

Statistic Shape Scale P-Value ARCH Lag[5] $0.00038890 .5002 .000 \quad 0.9843$ ARCH Lag[7] $0.00100051 .473 \quad 1.746 \quad 1.0000$ ARCH Lag[9] $0.00151382 .402 \quad 1.619 \quad 1.0000$

Nyblom stability test

Joint Statistic: 192.3745

Individual Statistics:

ar1 1.17129

ma1 1.21514

omega 13.94563

alpha1 1.66821

alpha2 0.05803

beta1 8.09970

beta2 6.96569

gamma1 13.50861

gamma2 3.15886

shape 9.79736

Asymptotic Critical Values (10\% 5\% 1\%)

Joint Statistic: 2.292 .543 .05

Individual Statistic: $\quad \begin{array}{llll}0.35 & 0.47 & 0.75\end{array}$

Sign Bias Test

$\begin{array}{lrr} & \text { t-value } & \text { prob sig } \\ \text { Sign Bias } & 0.95871 & 0.3378 \\ \text { Negative Sign Bias } & 0.48252 & 0.6295 \\ \text { Positive Sign Bias } & 0.05428 & 0.9567\end{array}$




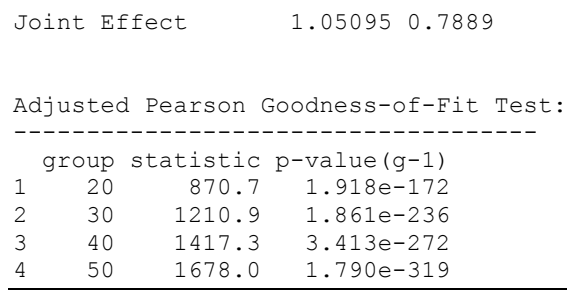

Table 17: Estimates of ARMA(1,1)-eGARCH(2,2) with sstd

\begin{tabular}{|c|c|c|c|c|}
\hline \multicolumn{4}{|c|}{ * GARCH Model Fit * } & \\
\hline \multicolumn{5}{|c|}{ 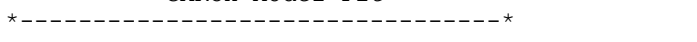 } \\
\hline \multicolumn{5}{|c|}{ Conditional Variance Dynamics } \\
\hline \multicolumn{5}{|c|}{ 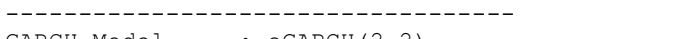 } \\
\hline \multicolumn{2}{|c|}{ GARCH Model : } & \\
\hline \multicolumn{2}{|c|}{ Mean Model } & \multicolumn{3}{|c|}{$\operatorname{ARFIMA}(1,0,1)$} \\
\hline \multicolumn{2}{|c|}{ Distribution } & \multicolumn{2}{|c|}{ sstd } & \\
\hline \multicolumn{5}{|c|}{ Optimal Parameters } \\
\hline \multicolumn{5}{|c|}{----------------------------} \\
\hline & Estimate & Std. Error & t value & $e \operatorname{Pr}(>|t|)$ \\
\hline $\operatorname{ar} 1$ & 0.311104 & 0.018626 & 16.7028 & 80.000000 \\
\hline $\operatorname{ma1}$ & 0.031624 & 0.013750 & 2.2999 & 90.02145 \\
\hline omega & -0.428565 & 0.023080 & -18.5689 & \\
\hline alpha1 & 0.859577 & 0.148896 & 5.7730 & 0.00000 \\
\hline alpha2 & 1.059502 & 0.134331 & 7.8872 & \\
\hline betal & 0.031654 & 0.001147 & 27.5924 & \\
\hline beta2 & 0.926006 & 0.001006 & 920.5848 & \\
\hline gamma 1 & 10.000000 & 22874 & 437.1 & \\
\hline gamma 2 & 9.399735 & 72643 & 129.39 & \\
\hline skew & 1.005109 & 0.009240 & 108.78 & \\
\hline shape & 2.010257 & 0.000173 & 11597.2175 & \\
\hline \multicolumn{5}{|c|}{ Robust Standard Errors: } \\
\hline & Estimate & Std. Error & t value & $\operatorname{Pr}(>|t|)$ \\
\hline $\operatorname{ar} 1$ & 0.311104 & 0.049329 & 6.3068 & 0.00 \\
\hline ma1 & 0.031624 & 0.020087 & 1.5743 & 0.11542 \\
\hline omega & -0.428565 & 0.150406 & -2.8494 & 0.00438 \\
\hline alphal & 0.859577 & 0.774569 & 1.1097 & 0.26711 \\
\hline alpha2 & 1.059502 & 0.747112 & 1.4181 & 0.15615 \\
\hline betal & 0.031654 & 0.004750 & 6.6637 & 0.00000 \\
\hline beta2 & 0.926006 & 0.002727 & 339.5184 & 0.00000 \\
\hline gamma 1 & 10.000000 & 1.405909 & 7.1128 & 0.00000 \\
\hline gamma 2 & 9.399735 & 1.282506 & 7.3292 & 0.00000 \\
\hline skew & 1.005109 & 0.006673 & 150.6302 & 0.00000 \\
\hline shape & 2.010257 & 0.000824 & 2438.2258 & 0.00000 \\
\hline
\end{tabular}

LogLikelihood : 10318.96

Information Criteria

$\begin{array}{ll} & \\ \text { Akaike } & -5.1334 \\ \text { Bayes } & -5.1162 \\ \text { Shibata } & -5.1335 \\ \text { Hannan-Quinn } & -5.1273\end{array}$

Weighted Ljung-Box Test on Standardized Residuals

statistic p-value
O.006631 0.9795

Lag[1] 0.00066310 .9795

$\operatorname{Lag}[2 *(p+q)+(p+q)-1][5] \quad 0.0019914 \quad 1.0000$

$\operatorname{Lag}[4 *(p+q)+(p+q)-1][9] \quad 0.0033223 \quad 1.0000$

d. $\circ \cdot f=2$

HO : No serial correlation

Weighted Ljung-Box Test on Standardized Squared Residuals

$\begin{array}{llr} & \text { statistic } & p-v a l u e \\ \operatorname{Lag}[1] & 0.0003884 & 0.9843 \\ \left.\operatorname{Lag}\left[2^{*}+q\right)+(p+q)-1\right][11] & 0.0023365 & 1.0000\end{array}$

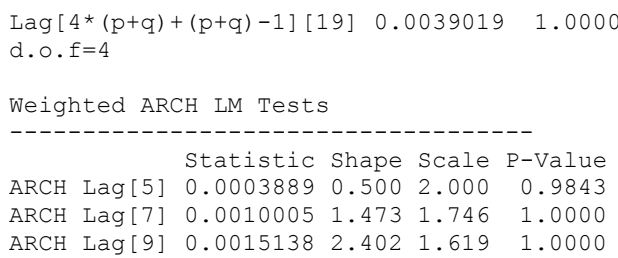

Table 18: Estimates of ARMA(1,1)-TGARCH(2,2) with std

\begin{tabular}{|c|c|c|c|c|}
\hline $\begin{array}{l}\star------1 \\
\star\end{array}$ & GARCH & * GARCH Model Fit * & $\begin{array}{r}-----{ }^{*} \\
\text { * }\end{array}$ & \\
\hline \multicolumn{5}{|c|}{ 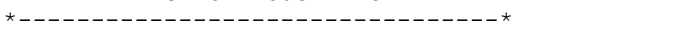 } \\
\hline \multicolumn{5}{|c|}{ Conditional Variance Dynamics } \\
\hline \multicolumn{5}{|c|}{ GARCH Model $\quad$ f fGARCH $(2,2)$} \\
\hline \multicolumn{5}{|c|}{ fGARCH Sub-Model : TGARCH } \\
\hline \multicolumn{5}{|c|}{ Mean Model : $\operatorname{ARFIMA}(1,0,1)$} \\
\hline \multicolumn{2}{|c|}{ Distribution } & : std & & \\
\hline \multicolumn{5}{|c|}{ Optimal Parameters } \\
\hline \multicolumn{5}{|c|}{ - } \\
\hline & Estimate & Std. Error & t value & \\
\hline $\operatorname{ar} 1$ & 0.188006 & 0.019754 & 9.51725 & \\
\hline ma1 & -0.044100 & 0.016926 & -2.60537 & \\
\hline omega & 0.000000 & 0.000000 & 0.56731 & \\
\hline alpha1 & 0.737207 & 0.022620 & 32.59082 & \\
\hline alpha2 & 0.007113 & 0.000221 & 32.23497 & \\
\hline beta1 & 0.391203 & 0.048539 & 8.05958 & 0. \\
\hline beta2 & 0.067454 & 0.031730 & 2.12587 & 0.033514 \\
\hline eta11 & -0.021401 & 0.022010 & -0.97233 & 0.330887 \\
\hline eta12 & -0.571818 & 0.013305 & -42.97626 & 0.000000 \\
\hline shape & 3.119393 & 0.058240 & 53.56058 & 0.000000 \\
\hline \multicolumn{5}{|c|}{ Robust Standard Errors: } \\
\hline & Estimate & Std. Error & t value & $\operatorname{Pr}(>|t|)$ \\
\hline $\operatorname{ar} 1$ & 0.188006 & 0.361081 & 0.520676 & 0.602593 \\
\hline ma1 & -0.044100 & 0.019349 & -2.279187 & 0.022656 \\
\hline
\end{tabular}




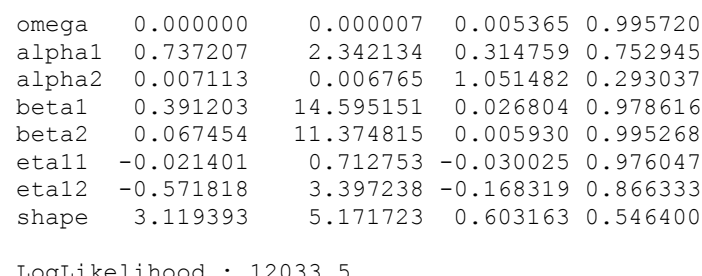

LogLikelihood : 12033.5

$\begin{array}{ll}\text { Information Criteria } \\ \text { Akaike } & -5.9878 \\ \text { Bayes } & -5.9721 \\ \text { Shibata } & -5.9878 \\ \text { Hannan-Quinn } & -5.9822\end{array}$

Weighted Ljung-Box Test on Standardized Residuals

$\begin{array}{lll} & \text { statistic } & p-v a l u e \\ \operatorname{Lag}[1] & 6.448 e-09 & 0.9999 \\ \operatorname{Lag}\left[2^{*}(p+q)+(p+q)-1\right][5] & 6.551 e-09 & 1.0000 \\ \operatorname{Lag}\left[4^{*}(p+q)+(p+q)-1\right][9] & 8.576 e-09 & 1.0000 \\ \text { d.o.f }=2 & \end{array}$

Weighted Ljung-Box Test on Standardized Squared Residuals

$\begin{array}{lrr}\operatorname{Lag}[1] & \text { statistic } & p-v a l u e \\ \operatorname{Lag}\left[2^{*}(p+q)+(p+q)-1\right][11] & 0.001951 & 0.9648 \\ \operatorname{Lag}[4 *(p+q)+(p+q)-1][19] & 0.019599 & 1.0000 \\ \text { d. } \circ . f=4 & & \end{array}$

d. o. $f=4$

Weighted ARCH LM Tests

Statistic Shape Scale P-Value

$\begin{array}{llllll}A R C H & \text { Lag[5] } 0.001954 & 0.500 & 2.000 & 0.9647\end{array}$

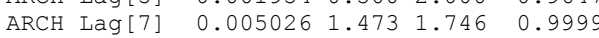

$\begin{array}{llllll}A R C H & \operatorname{Lag}[9] & 0.007604 & 2.402 & 1.619 & 1.0000\end{array}$

Nyblom stability test

Joint Statistic: 287.706

Individual Statistics:

$\operatorname{arl} \quad 0.2268$

ma1 0.1519

omega 124.3815

alpha1 69.1607

alpha2 7.1574

betal 11.0448

beta2 6.4155

eta11 0.8532

eta12 6.4544

shape $\quad 3.2976$

Asymptotic Critical Values (10\% 5\% 1\%) Joint Statistic: $\quad 2.292 .543 .05$

Individual Statistic: $\quad \begin{array}{llll}0.35 & 0.47 & 0.75\end{array}$

Sign Bias Test

$\begin{array}{lrl} & \text { t-value } & \text { prob sig } \\ \text { Sign Bias } & 0.002147 & 0.9983 \\ \text { Negative Sign Bias } & 0.384569 & 0.7006 \\ \text { Positive Sign Bias } & 0.377895 & 0.7055 \\ \text { Joint Effect } & 0.290821 & 0.9617\end{array}$

Adjusted Pearson Goodness-of-Fit Test:

\begin{tabular}{rrrr} 
& group statistic & p-value $(g-1)$ \\
1 & 20 & 926.9 & $2.054 \mathrm{e}-184$ \\
3 & 30 & 1253.4 & $1.733 e-245$ \\
4 & 40 & 1511.5 & $3.960 \mathrm{e}-292$ \\
\hline
\end{tabular}

Table 19: Estimates of ARMA(1,1)-TGARCH(2,2) with sstd

\begin{tabular}{|c|c|c|c|c|}
\hline \multicolumn{5}{|c|}{ 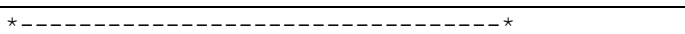 } \\
\hline \multicolumn{5}{|c|}{ * GARCH Model Fit * } \\
\hline \multicolumn{5}{|c|}{ 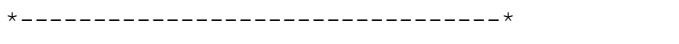 } \\
\hline \multicolumn{5}{|c|}{ Conditional Variance Dynamics } \\
\hline \multicolumn{5}{|c|}{ GARCH Model : ffGARCH $(2,2)$} \\
\hline \multicolumn{5}{|c|}{ fGARCH Sub-Model : TGARCH } \\
\hline \multicolumn{5}{|c|}{ Mean Model : $\operatorname{ARFIMA}(1,0,1)$} \\
\hline \multicolumn{2}{|c|}{ Distribution } & : sstd & & \\
\hline \multicolumn{5}{|c|}{ Optimal Parameters } \\
\hline \multicolumn{5}{|c|}{--- - - - - - - - - - - - - - - - - - - - - - - - - - - - - - - - - } \\
\hline $\operatorname{ar1}$ & 0.180819 & 0.027211 & 6.64515 & 0.000000 \\
\hline ma1 & -0.040789 & 0.027805 & -1.46695 & 0.142391 \\
\hline omega & 0.000000 & 0.000000 & 0.25422 & 0.799323 \\
\hline alpha1 & 0.751149 & 0.019581 & 38.36156 & 0.000000 \\
\hline alpha2 & 0.021083 & 0.000863 & 24.42460 & 0.000000 \\
\hline beta 1 & 0.338048 & 0.034700 & 9.74188 & 0.000000 \\
\hline beta2 & 0.099881 & 0.023224 & 4.30084 & 0.000017 \\
\hline eta11 & -0.032566 & 0.022312 & -1.45958 & 0.144405 \\
\hline eta12 & -0.009352 & 0.036362 & -0.25720 & 0.797028 \\
\hline skew & 1.008252 & 0.011963 & 84.28353 & 0.000000 \\
\hline shape & 3.119786 & 0.059674 & 52.28027 & 0.000000 \\
\hline \multicolumn{5}{|c|}{ Robust Standard Errors: } \\
\hline & Estimate & Std. Error & t value & $\operatorname{Pr}(>|t|)$ \\
\hline $\operatorname{ar1}$ & 0.180819 & 0.400435 & 0.451555 & 0.65159 \\
\hline ma1 & -0.040789 & 0.065709 & -0.620747 & 0.5347 \\
\hline omega & 0.000000 & 0.000032 & 0.001112 & 0.9991 \\
\hline alpha1 & 0.751149 & 0.749862 & 1.001716 & \\
\hline alpha2 & 0.021083 & 0.006765 & 3.116443 & 0.00183 \\
\hline beta 1 & 0.338048 & 6.615345 & 0.051101 & \\
\hline beta2 & 0.099881 & 6.442248 & 0.015504 & 0.9876 \\
\hline eta11 & -0.032566 & 0.527177 & -0.061775 & 0.9507 \\
\hline eta12 & -0.009352 & 1.470815 & -0.006359 & \\
\hline skew & 1.008252 & 0.063847 & 15.791770 & 0.00000 \\
\hline shape & 3.119786 & 3.512696 & 0.888146 & 0.37446 \\
\hline
\end{tabular}

LogLikelihood : 12042.07

Information Criteria

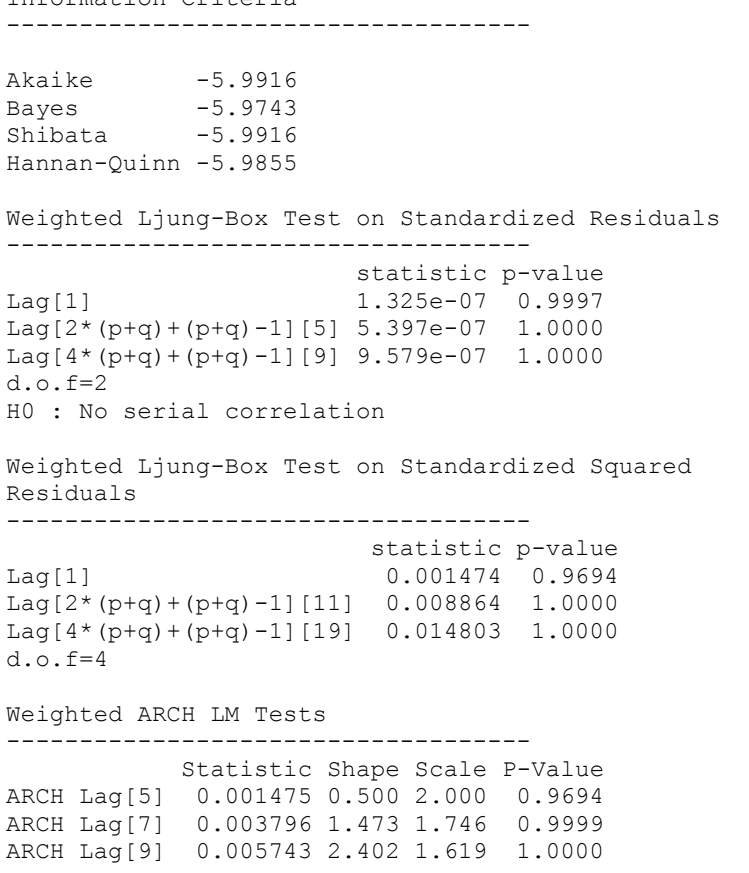


Nyblom stability test

Joint Statistic: 275.0765

Individual Statistics:

$\operatorname{arl} \quad 0.2426$

ma1 0.1458

omega 120.3569

alpha1 59.1178

alpha2 5.9049

beta1 8.7332

beta2 5.8779

eta11 1.0777

eta12 6.0758

skew 0.1025

shape $\quad 3.6370$

Asymptotic Critical Values (10\% 5\% 1\%)

Joint Statistic: $\quad 2.492 .75 \quad 3.27$

Individual Statistic: $\quad 0.350 .470 .75$

Sign Bias Test

$\begin{array}{lrr} & \text { t-value } & \text { prob sig } \\ \text { Sign Bias } & 0.1533 & 0.8782 \\ \text { Negative Sign Bias } & 0.3937 & 0.6938 \\ \text { Positive Sign Bias } & 0.3207 & 0.7484 \\ \text { Joint Effect } & 0.2768 & 0.9643\end{array}$

Adjusted Pearson Goodness-of-Fit Test:

$\begin{array}{rrrr} & \text { group } & \text { statistic } & p \text {-value }(g-1) \\ 1 & 20 & 957.9 & 5.097 e-191 \\ 2 & 30 & 1265.1 & 5.619 e-248 \\ 3 & 40 & 1599.4 & 9.439 e-311 \\ 4 & 50 & 1889.7 & 0.000 e+00\end{array}$

Table 20: Estimates of ARMA(1,1)-NAGARCH(1,1) with std

\begin{tabular}{|c|c|c|c|c|}
\hline \multirow{2}{*}{\multicolumn{5}{|c|}{$\begin{array}{ll}\star & \text { GARCH Model Fit } \\
\star & *\end{array}$}} \\
\hline & & & & \\
\hline \multicolumn{5}{|c|}{ Conditional Variance Dynamics } \\
\hline GARCH Model : fGARCH $(2,2)$ & \multicolumn{4}{|c|}{----------------------------------- } \\
\hline \multicolumn{5}{|c|}{ fGARCH Sub-Model : NAGARCH } \\
\hline \multicolumn{5}{|c|}{ Mean Model $\quad: \operatorname{ARFIMA}(1,0,1)$} \\
\hline \multicolumn{5}{|c|}{ Distribution } \\
\hline \multicolumn{5}{|c|}{ Optimal Parameters } \\
\hline \multicolumn{5}{|c|}{ 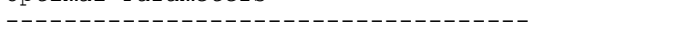 } \\
\hline & Estimate & Std. Error & t value & $\operatorname{Pr}(>|t|)$ \\
\hline $\operatorname{ar1}$ & 0.267628 & 0.170384 & 1.57074 & 0.116244 \\
\hline ma1 & -0.204195 & 0.180250 & -1.13284 & 0.257281 \\
\hline omega & 0.000000 & 0.000000 & 0.12858 & 0.897688 \\
\hline alpha1 & 0.361676 & 0.018067 & 20.01888 & 00 \\
\hline alpha2 & 0.027698 & 0.009210 & 3.00738 & 635 \\
\hline beta1 & 0.370490 & 0.081133 & 4.56642 & 05 \\
\hline beta2 & 0.216297 & 0.058472 & 3.69914 & 16 \\
\hline eta21 & 0.056881 & 0.058199 & 0.97736 & 92 \\
\hline eta22 & 0.367374 & 0.021812 & 16.84251 & 0.000000 \\
\hline shape & 3.725628 & 0.107154 & 34.76898 & 0.000000 \\
\hline \multicolumn{5}{|c|}{ Robust Standard Errors: } \\
\hline & Estimate & Std. Error & t value & $\operatorname{Pr}(>|t|)$ \\
\hline $\operatorname{ar} 1$ & 0.267628 & 0.652530 & 0.410138 & $3 \quad 0.68170$ \\
\hline ma1 & -0.204195 & 0.564150 & -0.361951 & 0.71739 \\
\hline omega & 0.000000 & 0.000060 & 0.000595 & 0.99952 \\
\hline alphal & 0.361676 & 0.726879 & 0.497574 & 0.61878 \\
\hline alpha2 & 0.027698 & 0.068116 & 0.406631 & 0.68428 \\
\hline betal & 0.370490 & 7.019245 & 0.052782 & 0.95791 \\
\hline beta2 & 0.216297 & 6.860580 & 0.031528 & 0.97485 \\
\hline eta21 & 0.056881 & 0.198935 & 0.285929 & 0.77493 \\
\hline eta22 & 0.367374 & 1.733692 & 0.211903 & 0.83218 \\
\hline shape & 3.725628 & 3.399272 & 1.096008 & 0.27308 \\
\hline
\end{tabular}

LogLikelihood : 10171.79

$\begin{array}{ll}\text { Information Criteria } \\ \text { Akaike } & -5.0607 \\ \text { Bayes } & -5.0450 \\ \text { Shibata } & -5.0607 \\ \text { Hannan-Quinn } & -5.0551\end{array}$

Weighted Ljung-Box Test on Standardized Residuals - - - - - - - -

statistic p-value

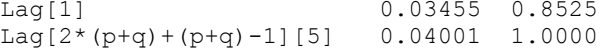

$\operatorname{Lag}[4 *(p+q)+(p+q)-1][9] \quad 0.06476 \quad 1.0000$

$\mathrm{d} . \mathrm{o} \cdot \mathrm{f}=2$

$\mathrm{HO}$ : No serial correlation

Weighted Ljung-Box Test on Standardized Squared Residuals

$\begin{array}{lrr} & \text { statistic } & p \text {-value } \\ \operatorname{Lag}[1] & 0.002135 & 0.9631 \\ \operatorname{Lag}\left[2^{*}(p+q)+(p+q)-1\right][11] & 0.014612 & 1.0000 \\ \operatorname{Lag}[4 *(p+q)+(p+q)-1][19] & 0.024386 & 1.0000 \\ \text { d.o. } f=4 & & \end{array}$

Weighted ARCH LM Tests

$\begin{array}{llrlll} & & \text { Statistic } & \text { Shape } & \text { Scale } & \text { P-Value } \\ \text { ARCH Lag[5] } & 0.002531 & 0.500 & 2.000 & 0.9599 \\ \text { ARCH Lag[7] } & 0.006381 & 1.473 & 1.746 & 0.9998 \\ \text { ARCH Lag[9] } & 0.009663 & 2.402 & 1.619 & 1.0000\end{array}$

Nyblom stability test

Joint Statistic: 228.1006

Individual Statistics:

$\operatorname{arl} \quad 0.3208$

$\operatorname{ma1} \quad 0.3712$

omega 93.0616

alpha1 48.2432

alpha2 20.2380

betal 6.3303

beta2 5.2483

eta21 2.1330

eta22 4.9865

shape 4.1481

Asymptotic Critical Values (10\% 5\% 1\%) Joint Statistic: 2.292 .543 .05 Individual Statistic: $\quad \begin{array}{llll}0.35 & 0.47 & 0.75\end{array}$

Sign Bias Test

$\begin{array}{lrr} & \text { t-value } & \text { prob sig } \\ \text { Sign Bias } & 0.9311 & 0.3519 \\ \text { Negative Sign Bias } & 0.5031 & 0.6149 \\ \text { Positive Sign Bias } & 0.1984 & 0.8427 \\ \text { Joint Effect } & 1.1151 & 0.7734\end{array}$

Adjusted Pearson Goodness-of-Fit Test:

\begin{tabular}{rrrr} 
& group & statistic & p-value $(g-1)$ \\
1 & 20 & 1136 & $5.322 e-229$ \\
3 & 30 & 1467 & $6.164 e-291$ \\
4 & 40 & 1702 & $0.000 e+00$ \\
\hline
\end{tabular}

Table 21: Estimates of ARMA(1,1)-NAGARCH(2,2) with sstd

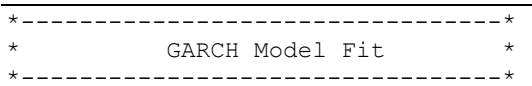


Conditional Variance Dynamics

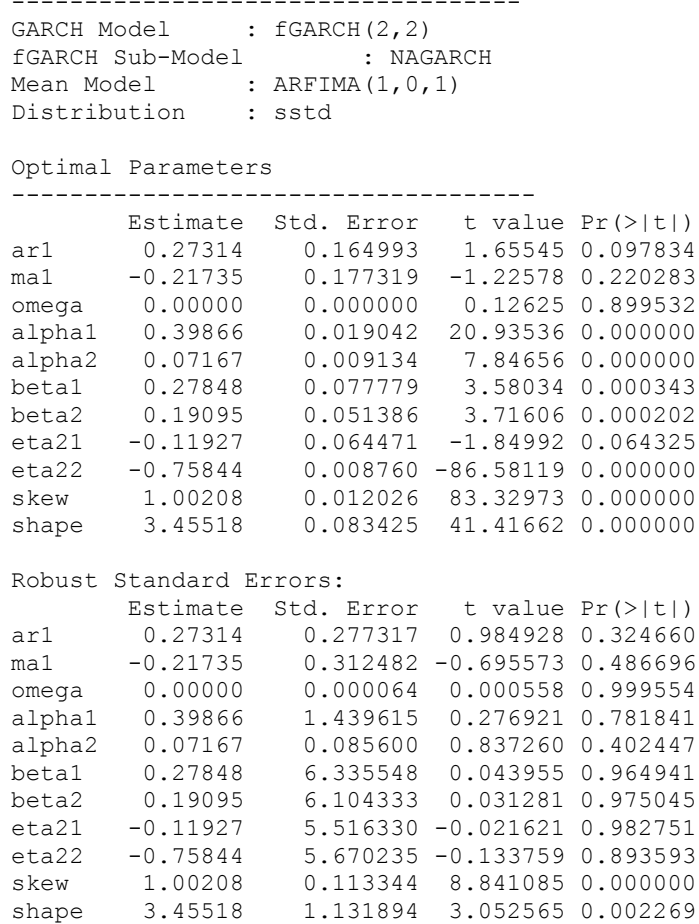

LogLikelihood : 10175.73

Information Criteria

$\begin{array}{ll}\text { Akaike } & -5.0621 \\ \text { Bayes } & -5.0449 \\ \text { Shibata } & -5.0621 \\ \text { Hannan-Quinn } & -5.0560\end{array}$

Weighted Ljung-Box Test on Standardized Residuals

$\begin{array}{lrr} & \text { Statistic } & p-v a l u e \\ \operatorname{Lag}[1] & 0.05674 & 0.8117 \\ \operatorname{Lag}\left[4^{*}(p+q)+(p+q)-1\right][5] & 0.06030 & 1.0000 \\ \text { d.o.f }=2 & 0.08085 & 1.0000 \\ \text { Ho : No serial correlation } & & \end{array}$

HO : No serial correlation

Weighted Ljung-Box Test on Standardized Squared Residuals

$\begin{array}{lrr} & \text { statistic } & p-v a l u e \\ \operatorname{Lag}[1] & 0.003088 & 0.9557 \\ \operatorname{Lag}\left[2^{*}(p+q)+(p+q)-1\right][11] & 0.020713 & 1.0000 \\ \operatorname{Lag}[4 *(p+q)+(p+q)-1][19] & 0.034493 & 1.0000 \\ \text { d.o.f }=4 & & \end{array}$

Weighted ARCH LM Tests

\begin{tabular}{|c|c|c|c|}
\hline & Statistic Shape & Scale & P-Value \\
\hline ARCH Lag [5] & 0.0035760 .500 & 2.000 & 0.9523 \\
\hline ARCH Lag[7] & 0.0090641 .473 & 1.746 & 0.9997 \\
\hline ARCH Lag [9] & 0.0136862 .402 & 1.619 & 1.0000 \\
\hline \multicolumn{4}{|c|}{ Nyblom stability test } \\
\hline \multirow{2}{*}{\multicolumn{4}{|c|}{$\begin{array}{l}\text { Joint Statistic: } 245.1376 \\
\text { Individual Statistics: }\end{array}$}} \\
\hline & & & \\
\hline \multicolumn{4}{|c|}{$\operatorname{arl} \quad 0.2838$} \\
\hline ma1 & 3408 & & \\
\hline omega 104. & 3987 & & \\
\hline alpha1 54. & 018 & & \\
\hline
\end{tabular}

$\begin{array}{ll}\text { alpha2 } & 8.7199 \\ \text { beta1 } & 8.7570 \\ \text { beta2 } & 6.9370 \\ \text { eta21 } & 0.7783 \\ \text { eta22 } & 3.9323 \\ \text { skew } & 0.1141 \\ \text { shape } & 3.4639\end{array}$

Asymptotic Critical Values (10\% 5\% $1 \frac{\circ}{\circ}$ ) Joint Statistic: $\quad 2.492 .753 .27$ Individual Statistic: $\quad 0.350 .470 .75$

Sign Bias Test

$\begin{array}{lrl} & \text { t-value prob sig } \\ \text { Sign Bias } & 0.7966 & 0.4257 \\ \text { Negative Sign Bias } & 0.5196 & 0.6034 \\ \text { Positive Sign Bias } & 0.2699 & 0.7873 \\ \text { Joint Effect } & 0.9490 & 0.8136\end{array}$

Adjusted Pearson Goodness-of-Fit Test:

\begin{tabular}{rrrr} 
& group statistic & p-value $(g-1)$ \\
2 & 20 & 1244 & $4.108 e-252$ \\
3 & 30 & 1611 & $1.047 e-321$ \\
4 & 40 & 1900 & $0.000 e+00$ \\
\hline
\end{tabular}

Table 22: Estimates of ARMA(1,1)-AVGARCH(2,2) with std

* GARCH Model Fit
*
*-1
Conditional Variance Dynamics
GARCH Model : fGARCH $(2,2)$
fGARCH Sub-Model $\quad:$ ARFIMA $(1,0,1)$
$\begin{aligned} & \text { Mean Model } \\ & \text { Distribution } \quad: \text { std }\end{aligned}$
Optimal Parameters

\begin{tabular}{|c|c|c|c|c|}
\hline & Estimate & Std. Error & $t$ value $P$ & $\operatorname{Pr}(>|t|)$ \\
\hline $\operatorname{ar} 1$ & 0.158970 & 0.015930 & 9.979590 & 0.000000 \\
\hline ma1 & -0.137234 & 0.019347 & -7.093440 & 0.000000 \\
\hline omega & 0.000000 & 0.000000 & 0.329070 & 0.742103 \\
\hline alphal & 0.736912 & 0.022551 & $32.67807 \quad 0$ & 0.000000 \\
\hline alpha2 & 0.005087 & 0.000510 & 9.975690 & 0.000000 \\
\hline betal & 0.374727 & 0.041856 & 8.95270 & 0.000000 \\
\hline beta2 & 0.063603 & 0.026411 & 2.408210 & 0.016031 \\
\hline eta11 & -0.045385 & 0.024043 & -1.887650 & 0.059073 \\
\hline eta12 & 0.709412 & 0.171149 & 4.144990 & 0.000034 \\
\hline eta21 & 0.000162 & 0.000961 & 0.168190 & 0.866435 \\
\hline eta22 & 8.347500 & 0.822924 & 10.143710 & 0.000000 \\
\hline shape & 3.088752 & 0.070739 & 43.664320 & 0.000000 \\
\hline Robust & \multicolumn{4}{|c|}{ Standard Errors: } \\
\hline & Estimate & Std. Error & t value & e $\operatorname{Pr}(>|t|)$ \\
\hline $\operatorname{ar} 1$ & 0.158970 & 0.099143 & 1.603451 & 0.108835 \\
\hline ma1 & -0.137234 & 0.077725 & -1.765629 & 90.077458 \\
\hline omega & 0.000000 & 0.000019 & 0.001899 & 0.998485 \\
\hline alpha1 & 0.736912 & 0.239248 & 3.080123 & 0.002069 \\
\hline alpha2 & 0.005087 & 0.000033 & 153.551376 & 0.000000 \\
\hline beta1 & 0.374727 & 10.078103 & 0.037182 & 0.970340 \\
\hline beta2 & 0.063603 & 8.950449 & 0.007106 & 0.994330 \\
\hline eta11 & -0.045385 & 0.877183 & -0.051739 & 0.958736 \\
\hline eta12 & 0.709412 & 0.067197 & 10.557231 & 0.000000 \\
\hline eta21 & 0.000162 & 0.001038 & 0.155686 & 0.876280 \\
\hline eta22 & 8.347500 & 0.913383 & 9.139099 & 0.000000 \\
\hline shape & 3.088752 & 5.353848 & 0.576922 & 20.563992 \\
\hline
\end{tabular}

LogLikelihood : 12082.03

Information Criteria 


$\begin{array}{ll}\text { Akaike } & -6.0110 \\ \text { Bayes } & -5.9922 \\ \text { Shibata } & -6.0110 \\ \text { Hannan-Quinn } & -6.0043\end{array}$

Weighted Ljung-Box Test on Standardized Residuals

$\begin{array}{lll} & \text { statistic } & p-v a l u e \\ \operatorname{Lag}[1] & 2.408 e-06 & 0.9988 \\ \operatorname{Lag}\left[2^{*}(p+q)+(p+q)-1\right][5] & 7.945 e-06 & 1.0000 \\ \operatorname{Lag}[4 *(p+q)+(p+q)-1][9] & 1.349 e-05 & 1.0000 \\ \text { d. } 0 . f=2 & & \end{array}$

HO : No serial correlation

Weighted Ljung-Box Test on Standardized Squared Residuals

$\begin{array}{lrr} & \text { Statistic } & p \text {-value } \\ \operatorname{Lag}[1] & 0.001238 & 0.9719 \\ \operatorname{Lag}\left[2^{*}(p+q)+(p+q)-1\right][11] & 0.007444 & 1.0000 \\ \operatorname{Lag}[4 *(p+q)+(p+q)-1][19] & 0.012432 & 1.0000 \\ \text { d. } 0 . f=4 & & \end{array}$

. $\mathrm{.} . \mathrm{f}=4$

Weighted ARCH LM Tests

$\begin{array}{rrrrr} & & \text { Statistic Shape Scale } & \text { P-Value } \\ \text { ARCH Lag[5] } & 0.001239 & 0.500 & 2.000 & 0.9719 \\ \text { ARCH Lag[7] } & 0.003188 & 1.473 & 1.746 & 0.9999 \\ \text { ARCH Lag[9] } & 0.004823 & 2.402 & 1.619 & 1.0000\end{array}$

Nyblom stability test

Joint Statistic: -1051.369

Individual Statistics:

ar1 1.4224

ma1 1.5537

omega 113.9610

alpha1 28.8623

alpha2 7.2632

beta1 5.1249

beta2 7.1273

eta11 1.9999

eta12 7.2619

eta21 0.5528

eta22 7.1282

shape 6.1527

Asymptotic Critical Values (10\% 5\% 1\%)

Joint Statistic: $\quad 2.692 .96 \quad 3.51$

Individual Statistic: $\quad \begin{array}{llll}0.35 & 0.47 & 0.75\end{array}$

Sign Bias Test

$\begin{array}{lrr} & \text { t-value } & \text { prob sig } \\ \text { Sign Bias } & 0.2294 & 0.8186 \\ \text { Negative Sign Bias } & 0.3973 & 0.6911 \\ \text { Positive Sign Bias } & 0.3141 & 0.7535 \\ \text { Joint Effect } & 0.3056 & 0.9590\end{array}$

Adjusted Pearson Goodness-of-Fit Test:

\begin{tabular}{rrrr} 
& group statistic & p-value $(g-1)$ \\
2 & 20 & 1040 & $1.372 e-208$ \\
3 & 30 & 1559 & $1.494 e-310$ \\
4 & 40 & 2088 & $0.000 e+00$ \\
\hline
\end{tabular}

Table 23: Estimates of ARMA(1,1)-AVGARCH(2,2) with sstd

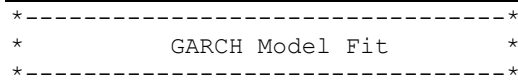

\begin{tabular}{|c|c|c|c|c|}
\hline \multicolumn{5}{|c|}{ GARCH Model : fGARCH $(2,2)$} \\
\hline \multicolumn{2}{|c|}{ Mean Model } & \multicolumn{3}{|c|}{$: \operatorname{ARFIMA}(1,0,1)$} \\
\hline \multicolumn{2}{|c|}{ Distribution } & \multicolumn{3}{|c|}{ : sstd } \\
\hline \multicolumn{5}{|c|}{ Optimal Parameters } \\
\hline \multicolumn{5}{|c|}{ 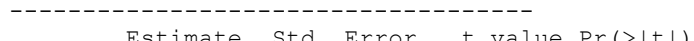 } \\
\hline & Estimate & Std. Error & t value & $\operatorname{Pr}(>|t|)$ \\
\hline 21 & 0.101731 & 0.024077 & 4.225306 & 0.000024 \\
\hline a1 & -0.001814 & 0.032550 & -0.055728 & 0.955558 \\
\hline mega & 0.000000 & 0.000000 & 0.213259 & 0.831125 \\
\hline lpha1 & 0.799645 & 0.018756 & 42.635197 & 0.000000 \\
\hline alpha2 & 0.008552 & 0.000802 & 10.663941 & 0.000000 \\
\hline etal & 0.273720 & 0.028337 & 9.659585 & 0.000000 \\
\hline eta2 & 0.046177 & 0.007158 & 6.450902 & 0.000000 \\
\hline $\operatorname{ta11}$ & -0.052875 & 0.024251 & -2.180361 & 29231 \\
\hline $\operatorname{ta} 12$ & 0.710317 & 0.160919 & 4.414136 & 010 \\
\hline $\operatorname{ta} 21$ & 0.158922 & 0.007346 & 21.634544 & 0000 \\
\hline ta22 & 9.417915 & 0.800430 & 11.766066 & 0.000000 \\
\hline ew & 1.003885 & 0.011379 & 88.224025 & 0.000000 \\
\hline hape & 2.822039 & 0.041927 & 67.308817 & 0.000000 \\
\hline \multicolumn{5}{|c|}{ Robust Standard Errors: } \\
\hline & Estimate & std. Error & t value & $\operatorname{Pr}(>|t|)$ \\
\hline 1 & 0.101731 & 1.067858 & 0.095266 & 0.924103 \\
\hline & -0.001814 & 1.465060 & -0.001238 & 0.999012 \\
\hline omega & 0.000000 & 0.000042 & 0.000854 & 0.999319 \\
\hline alphal & 0.799645 & 1.406233 & 0.568643 & 0.569598 \\
\hline alpha2 & 0.008552 & 0.000516 & 16.584686 & 0.000000 \\
\hline eta1 & 0.273720 & 4.509151 & 0.060703 & 0.951596 \\
\hline eta2 & 0.046177 & 4.998391 & 0.009238 & 0.992629 \\
\hline $\operatorname{ta11}$ & -0.052875 & 0.604364 & -0.087489 & 0.930283 \\
\hline $\operatorname{ta12}$ & 0.710317 & 1.239174 & 0.573218 & 0.566497 \\
\hline$a 21$ & 0.158922 & 0.556172 & 0.285742 & 0.775076 \\
\hline & 9.417915 & 2.795129 & 3.369403 & 0.000753 \\
\hline & 1.003885 & 0.044910 & 22.353023 & 0.000000 \\
\hline & 2.822039 & 1.009785 & 2.794693 & 0.005195 \\
\hline
\end{tabular}

LogLikelihood : 11900.28

Information Criteria

Akaike $\quad-5.9200$

Bayes $\quad-5.8996$

Shibata $\quad-5.9200$

Hannan-Quinn -5.9127

Weighted Ljung-Box Test on Standardized Residuals

$\begin{aligned} & \text { statistic } p-v a l u e\end{aligned}$

Lag [1] $\quad 5.637 e-060.9981$

$\operatorname{Lag}[2 *(p+q)+(p+q)-1][5] \quad 1.794 e-05 \quad 1.0000$

$\operatorname{Lag}\left[4^{*}(p+q)+(p+q)-1\right][9] \quad 3.022 e-05 \quad 1.0000$

d. $\circ \cdot f=2$

HO : No serial correlation

Weighted Ljung-Box Test on Standardized Squared Residuals

$\begin{array}{lrr} & \text { statistic } & p-\text { value } \\ \operatorname{Lag}[1] & 0.001205 & 0.9723 \\ \operatorname{Lag}\left[2^{*}(p+q)+(p+q)-1\right][11] & 0.007248 & 1.0000 \\ \operatorname{Lag}[4 *(p+q)+(p+q)-1][19] & 0.012105 & 1.0000 \\ \text { d.o.f=4 } & & \end{array}$

Weighted ARCH LM Tests

$\begin{array}{llrrrr} & & \text { Statistic Shape } & \text { Scale } & \text { P-Value } \\ \text { ARCH Lag[5] } & 0.001207 & 0.500 & 2.000 & 0.9723 \\ \text { ARCH Lag[7] } & 0.003104 & 1.473 & 1.746 & 0.9999 \\ \text { ARCH Lag[9] } & 0.004696 & 2.402 & 1.619 & 1.0000\end{array}$

Nyblom stability test

Joint Statistic: $\quad-1109.483$

Individual Statistics: 


$\begin{array}{lr}\text { ar1 } & 1.5266 \\ \text { ma1 } & 1.3519 \\ \text { omega } & 103.5352 \\ \text { alpha1 } & 16.0413 \\ \text { alpha2 } & 11.4688 \\ \text { beta1 } & 7.7769 \\ \text { beta2 } & 11.1323 \\ \text { eta11 } & 23.9089 \\ \text { eta12 } & 11.4688 \\ \text { eta21 } & 56.5608 \\ \text { eta22 } & 11.1304 \\ \text { skew } & 0.1852 \\ \text { shape } & 3.4749\end{array}$

Asymptotic Critical Values (10\% 5\% 1\%)

Joint Statistic: $\quad 2.893 .153 .69$

Individual Statistic: $\quad \begin{array}{llll}0.35 & 0.47 & 0.75\end{array}$

Sign Bias Test

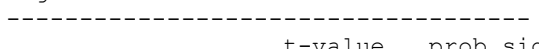

Sign Bias $\quad 0.22670 .8207$

Negative Sign Bias 0.39270 .6945

Positive Sign Bias 0.29510 .7679

Joint Effect $\quad 0.28650 .9625$

Adjusted Pearson Goodness-of-Fit Test:

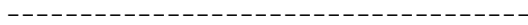

$\begin{array}{rrr}\text { group statistic } & \text { p-value }(g-1) \\ 20 & 1142 & 2.338 e-230\end{array}$

$230 \quad 1506 \quad 3.365 e-299$ 


\section{Appendix B}
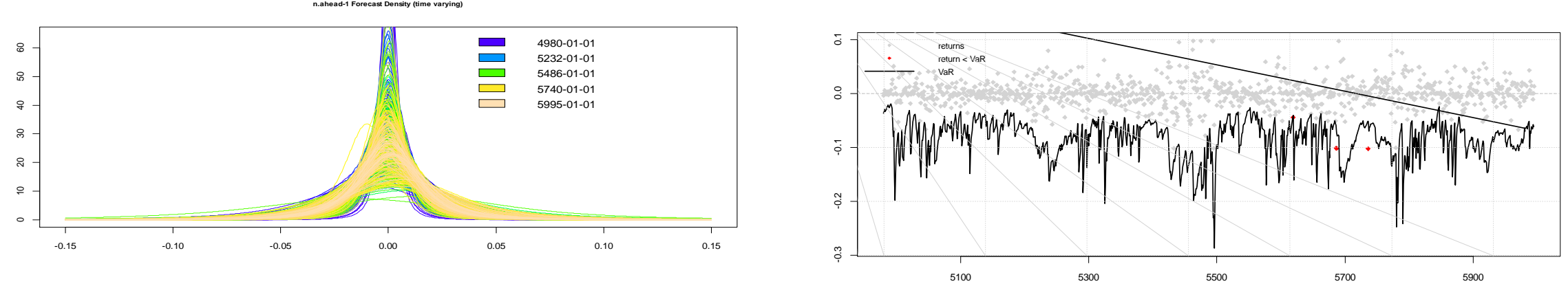

i

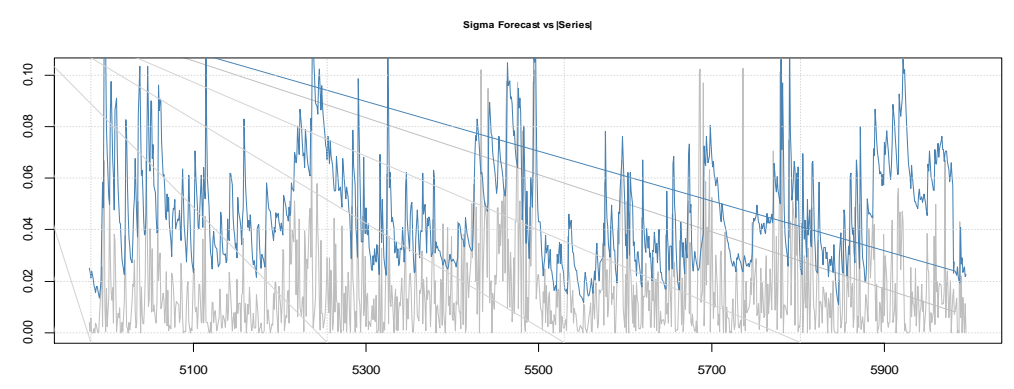

ii
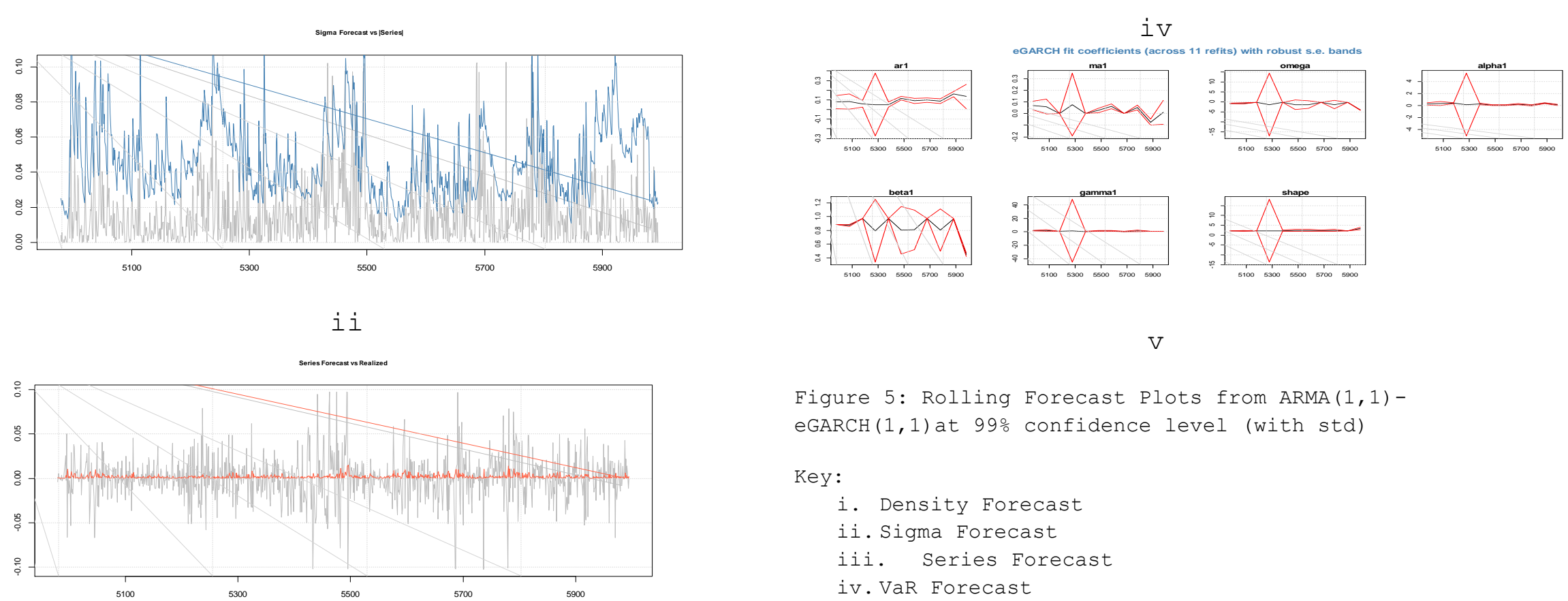

$\mathrm{V}$

Figure 5: Rolling Forecast Plots from ARMA $(1,1)$ $\operatorname{eGARCH}(1,1)$ at $99 \%$ confidence level (with std)

Key:

i. Density Forecast

ii. Sigma Forecast

iii. Series Forecast

iv. VaR Forecast

v. Fit Coefficients (with s.e band)

iii std: student $t$ distribution 


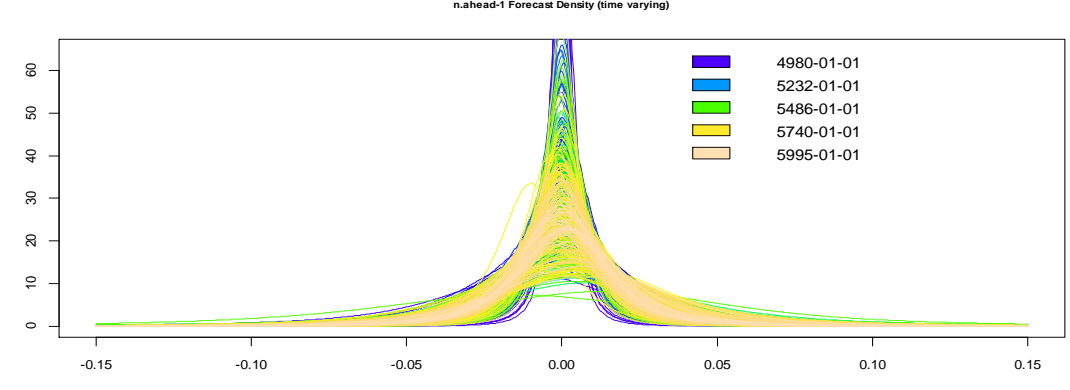

i

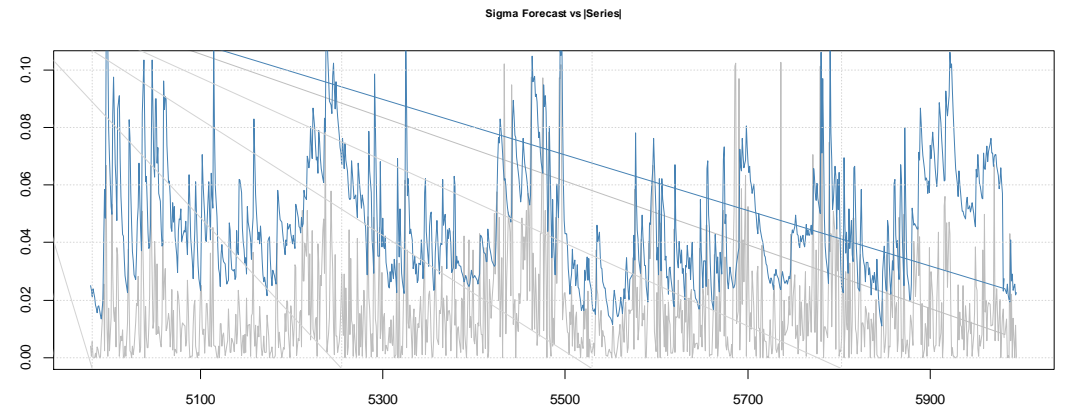

ii

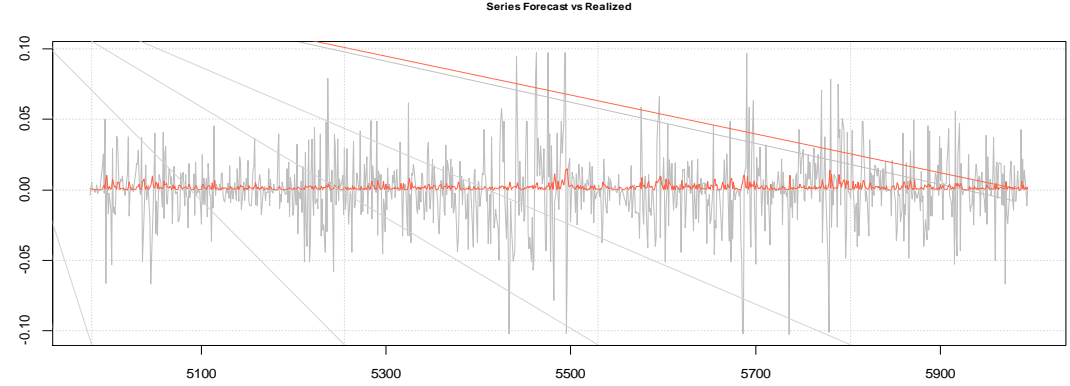

iii

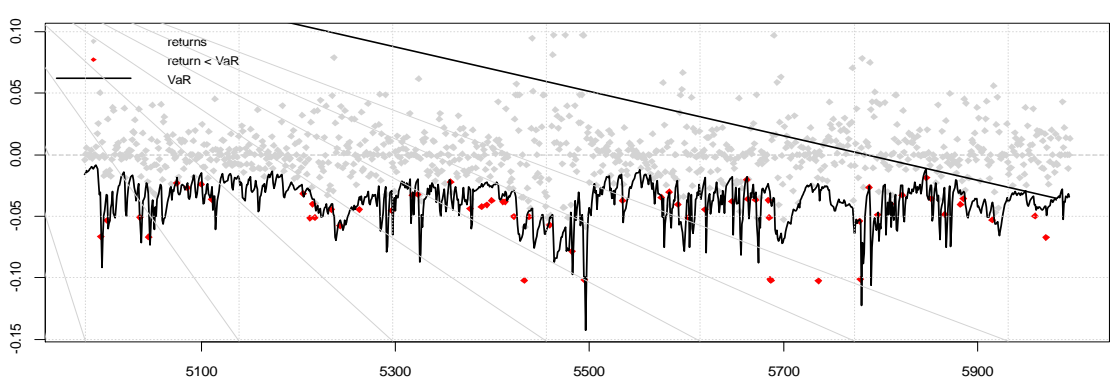

iv
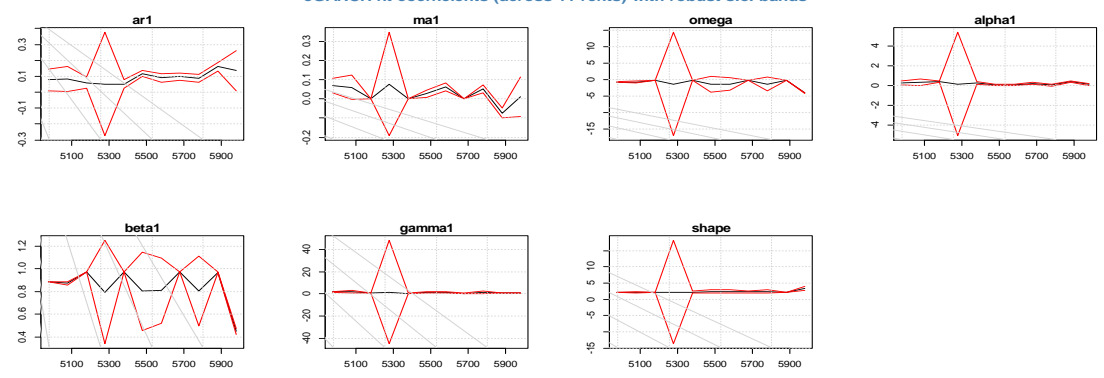

$\mathrm{V}$

Figure 6: Rolling Forecast Plots from ARMA $(1,1)-$ eGARCH $(1,1)$ at 95\% confidence level (with std)

Key:

i. Density Forecast

ii. Sigma Forecast

iii. Series Forecast

iv. VaR Forecast

v. Fit Coefficients (with s.e band) std: student $t$ distribution 


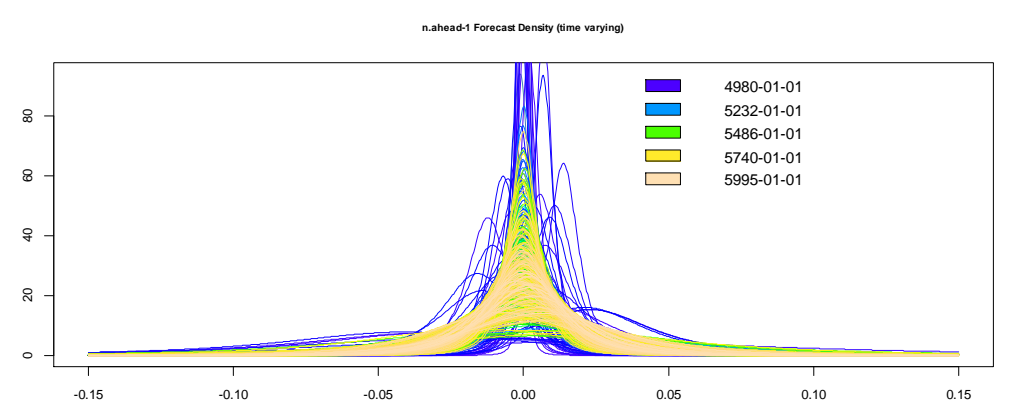

i

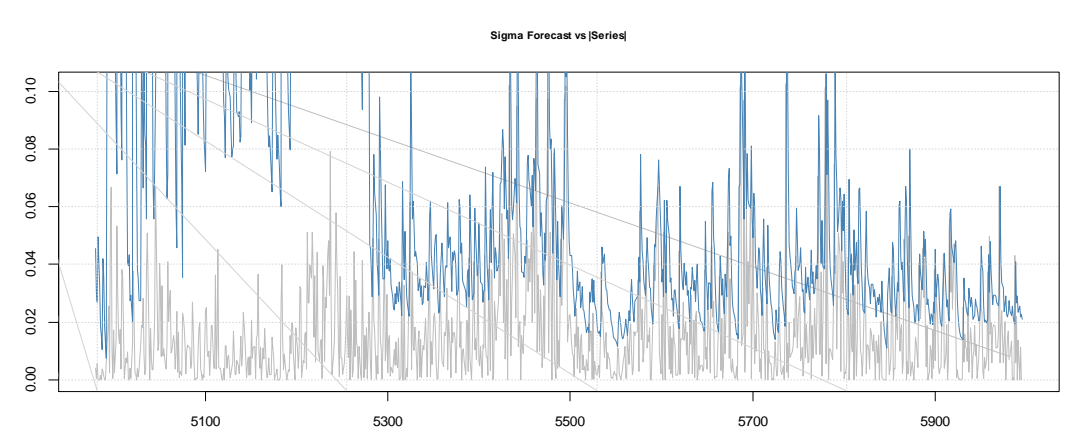

ii

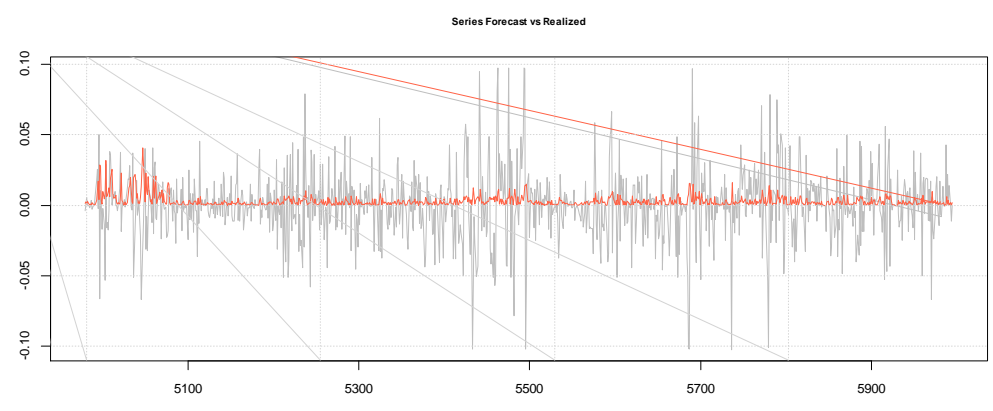

iii

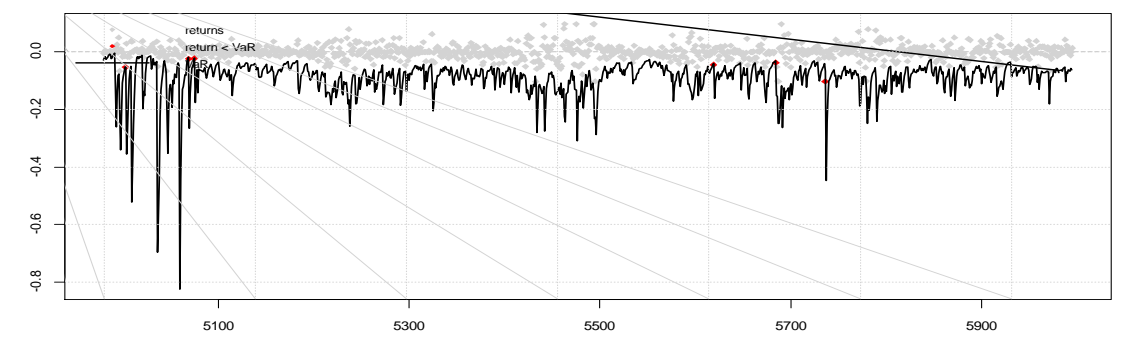

iv
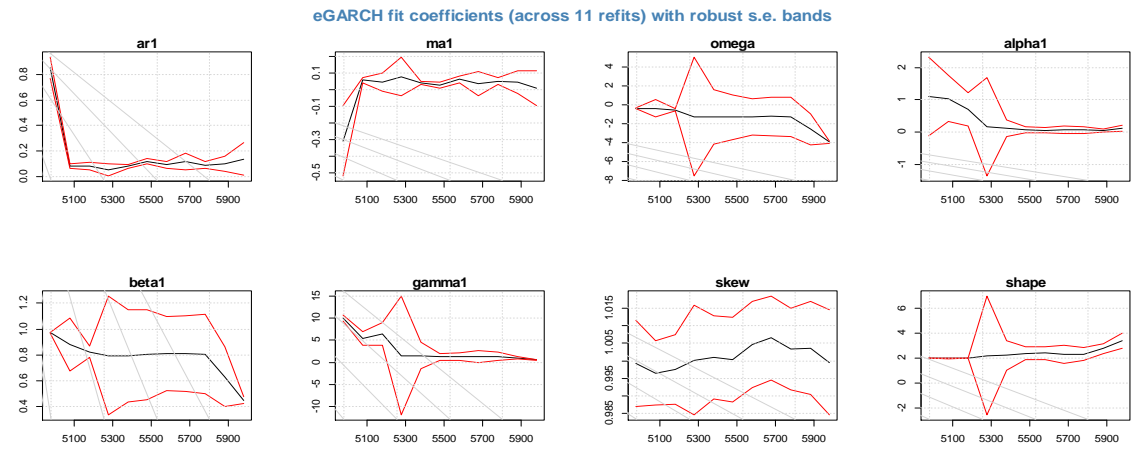

$\mathrm{V}$

Figure 7: Rolling Forecast Plots from ARMA $(1,1)$ $\operatorname{eGARCH}(1,1)$ at $99 \%$ confidence level (with sstd)

Key:

i. Density Forecast

ii. Sigma Forecast

iii. Series Forecast

iv. VaR Forecast

v. Fit Coefficients (with s.e band)

sstd: skewed student $t$ distribution 


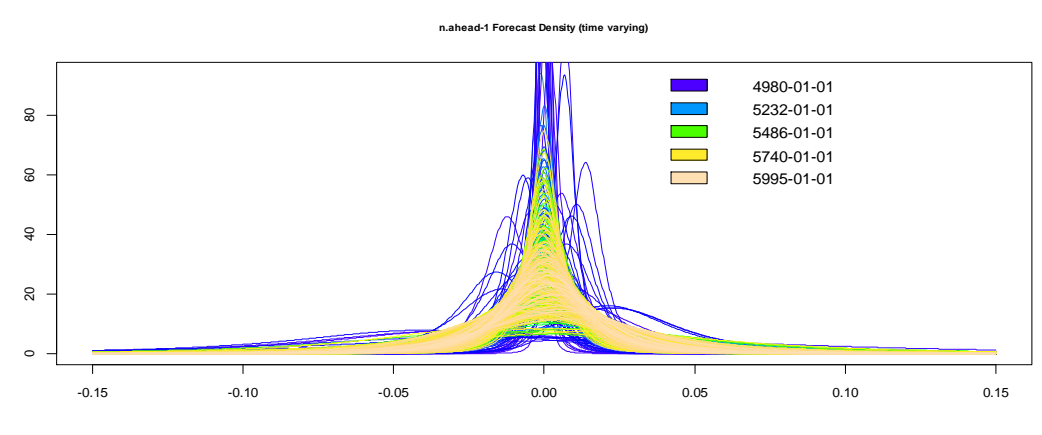

i

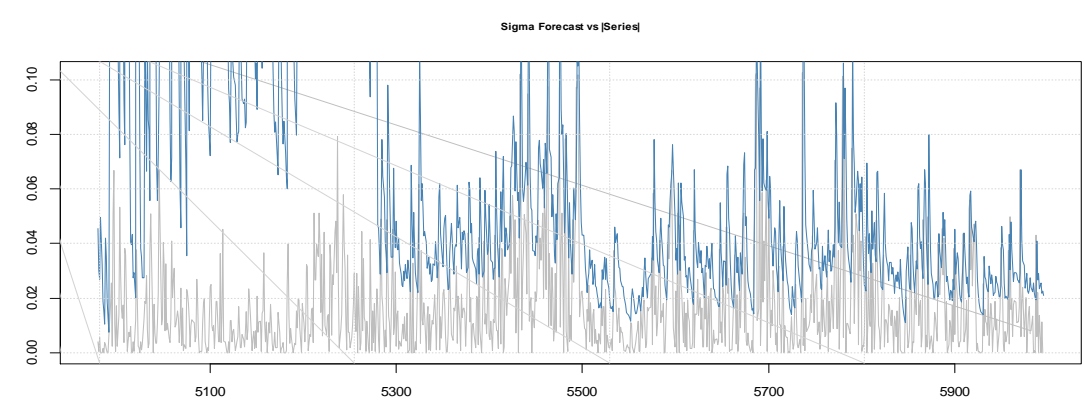

ii

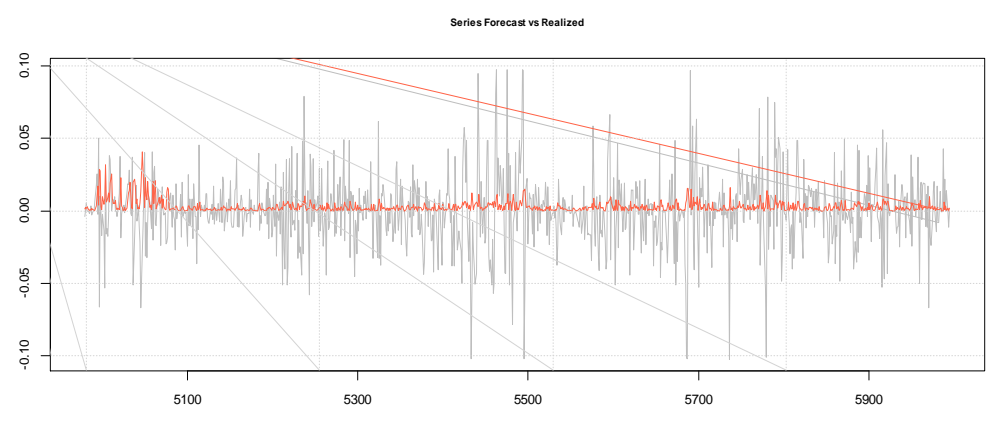

iii

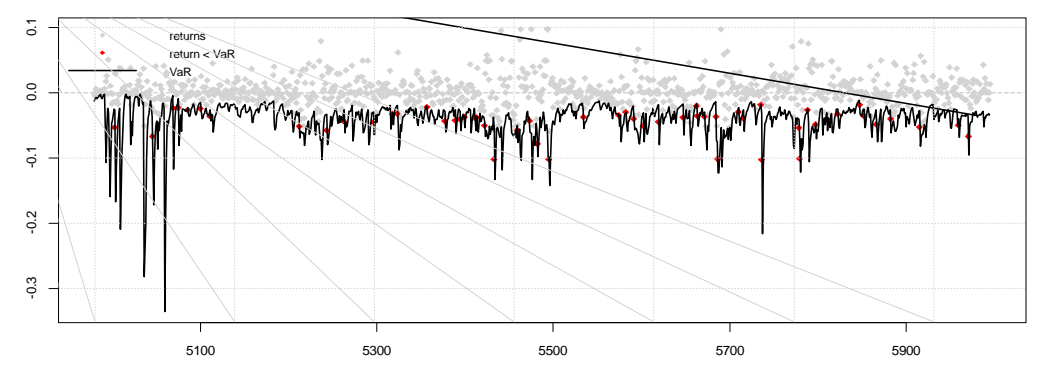

iv
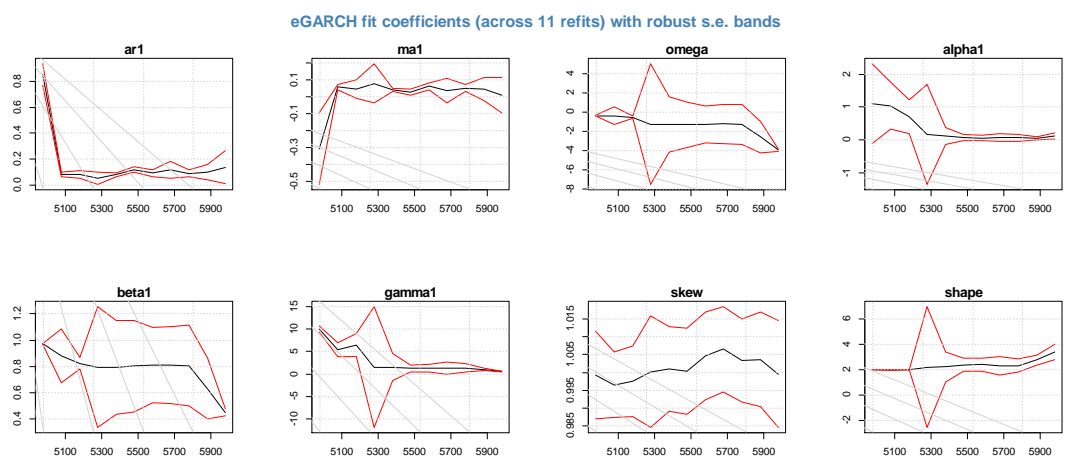

Figure 8: Rolling Forecast Plots from ARMA $(1,1)$ eGARCH $(1,1)$ at $95 \%$ confidence level (with sstd)

Key:

i. Density Forecast

ii. Sigma Forecast

iii. Series Forecast

iv. VaR Forecast

v. Fit Coefficients (with s.e band)

sstd: skewed student $t$ distribution 


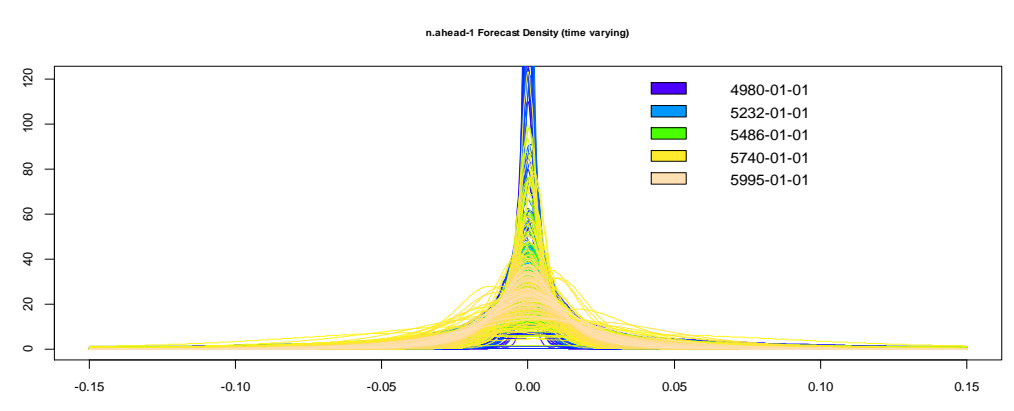

i

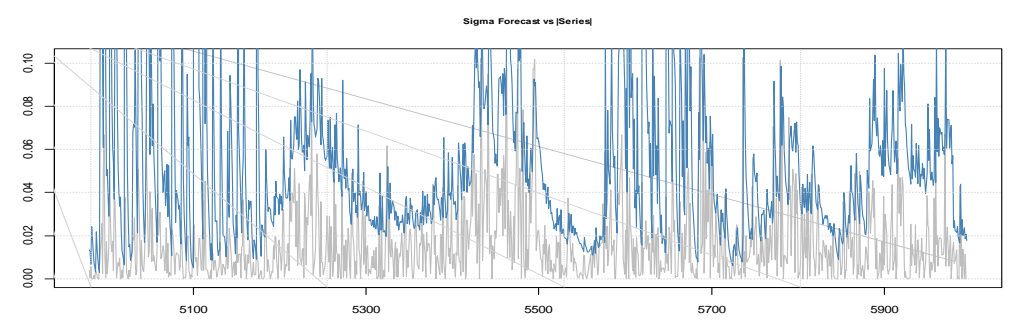

ii

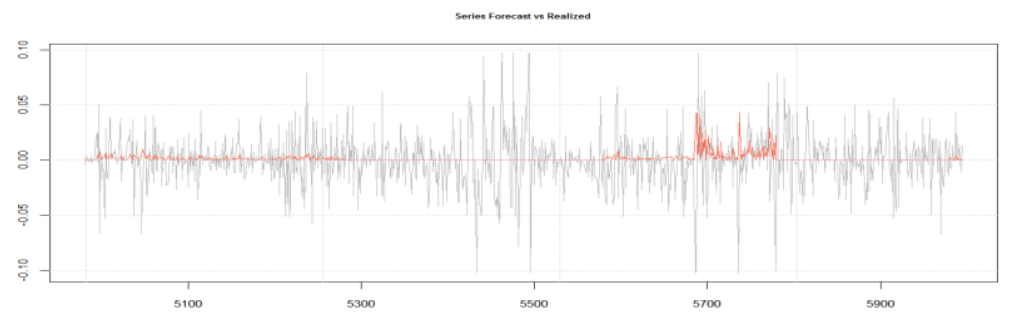

iii

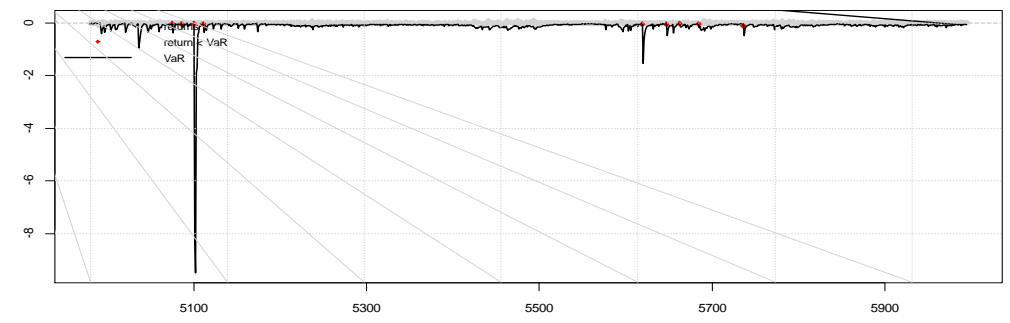

iv
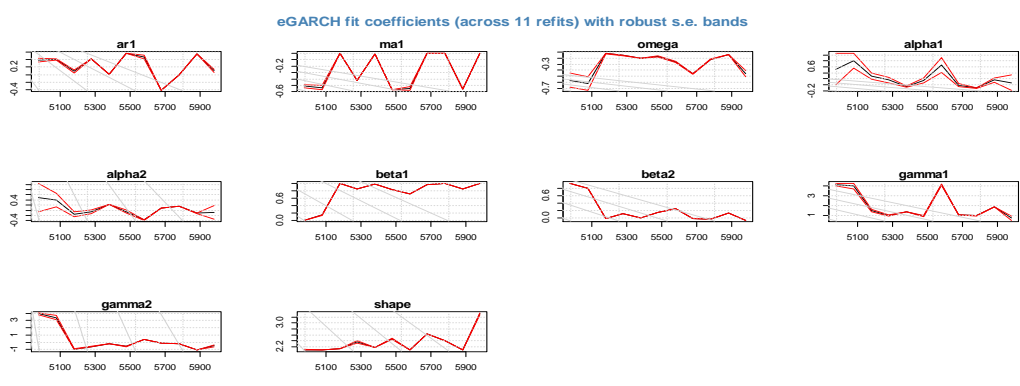

Figure 9: Rolling Forecast Plots from ARMA $(1,1)$ $\operatorname{eGARCH}(2,2)$ at $99 \%$ confidence level (with std)

Key:

i. Density Forecast

ii. Sigma Forecast

iii. Series Forecast

iv. VaR Forecast

v. Fit Coefficients (with s.e band)

std: student $t$ distribution 


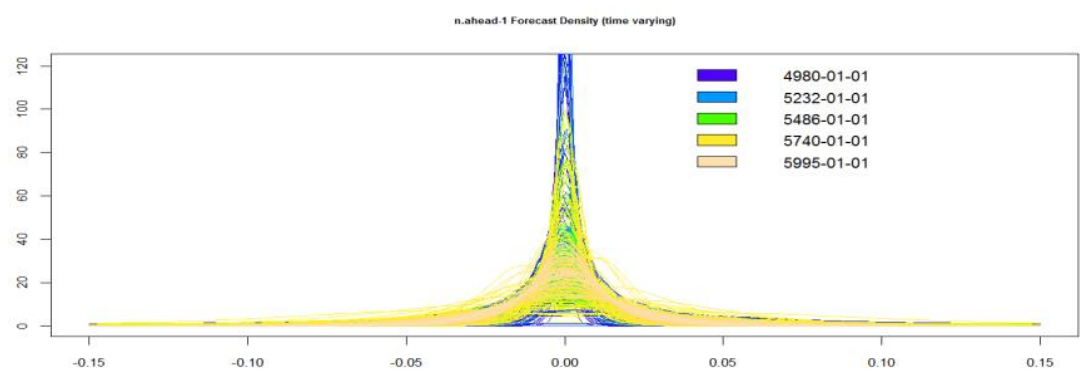

i

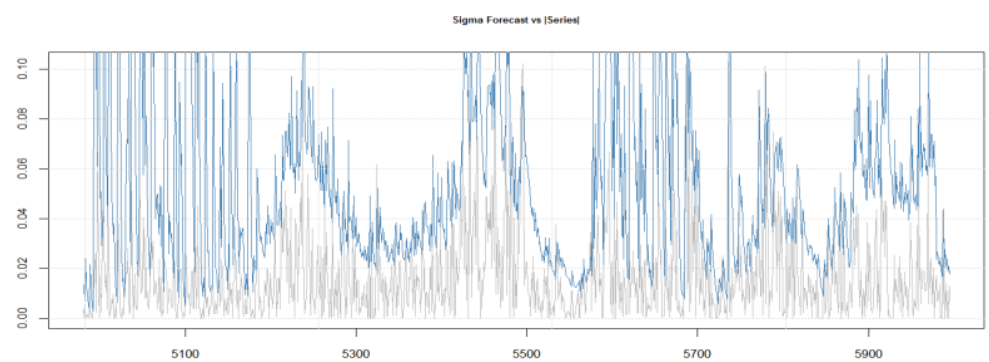

ii

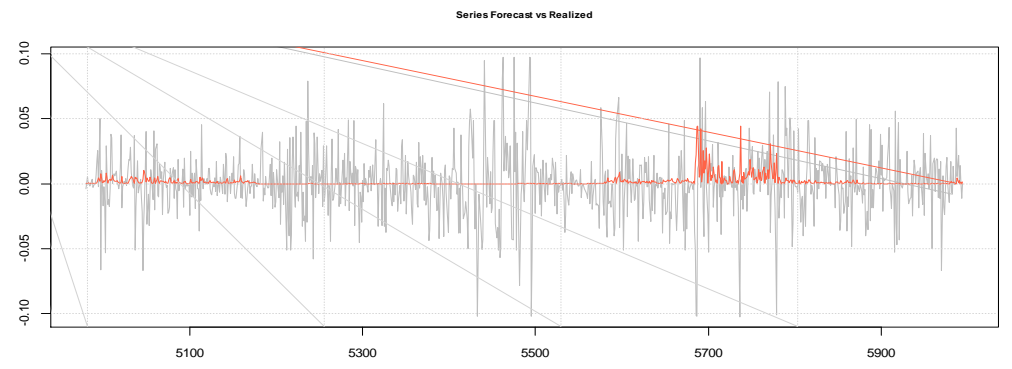

iii

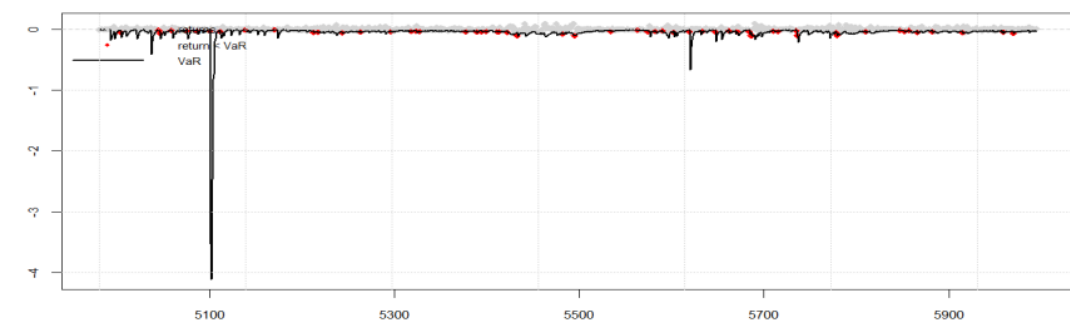

iv
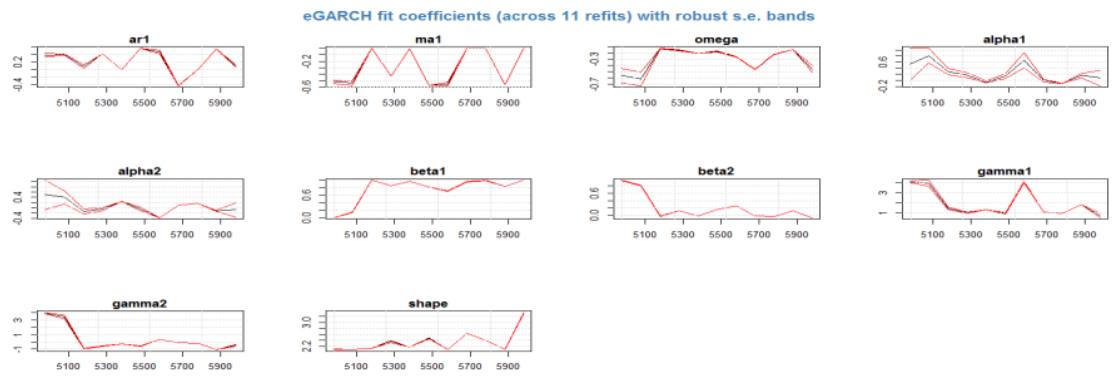

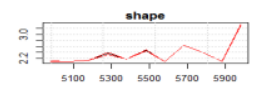

$\mathrm{V}$

Figure 10: Rolling Forecast Plots from $\operatorname{ARMA}(1,1)-$ eGARCH $(2,2)$ at 95\% confidence level (with std)

Key:

i. Density Forecast

ii. Sigma Forecast

iii. Series Forecast

iv. VaR Forecast

v. Fit Coefficients (with s.e band) std: student $t$ distribution 


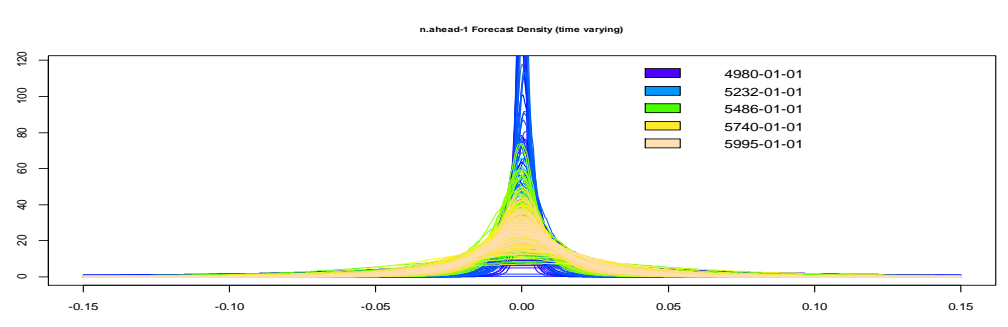

i

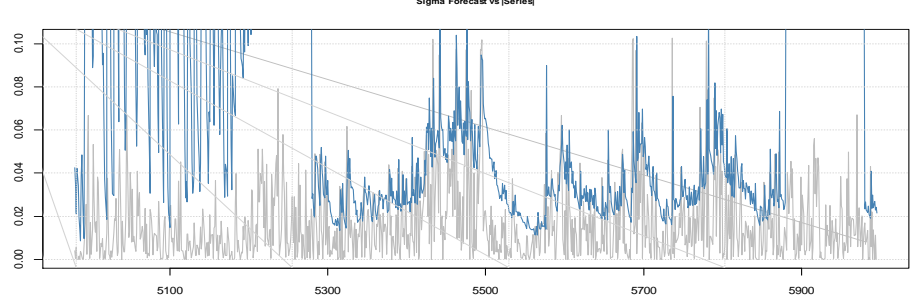

ii

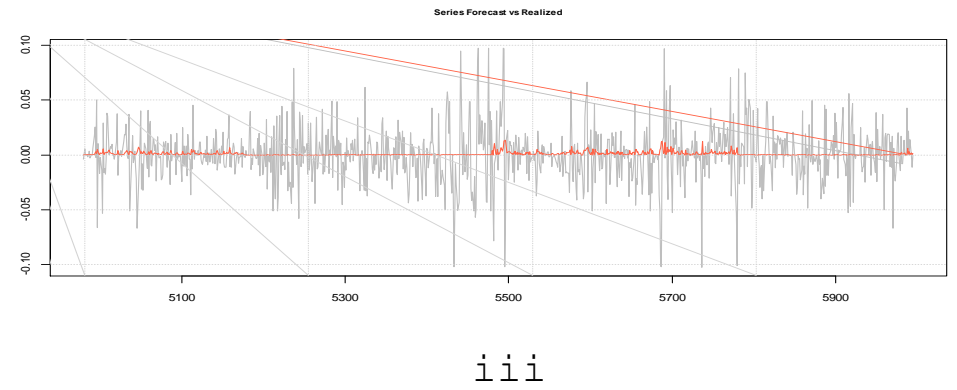

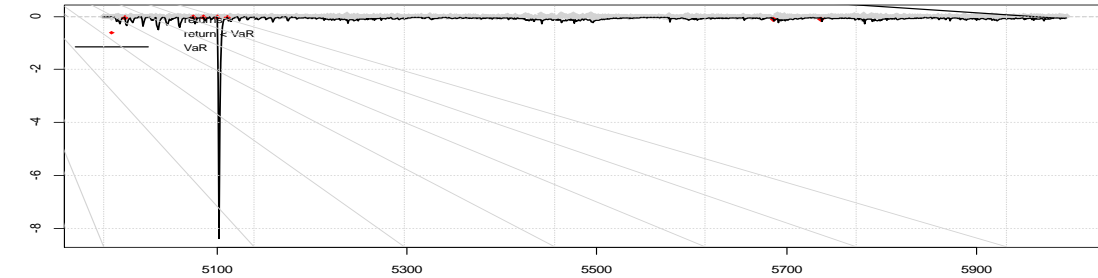

iv
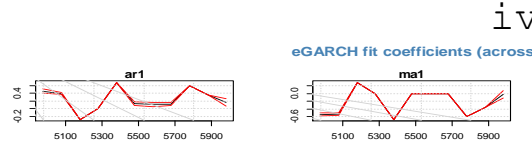

5.
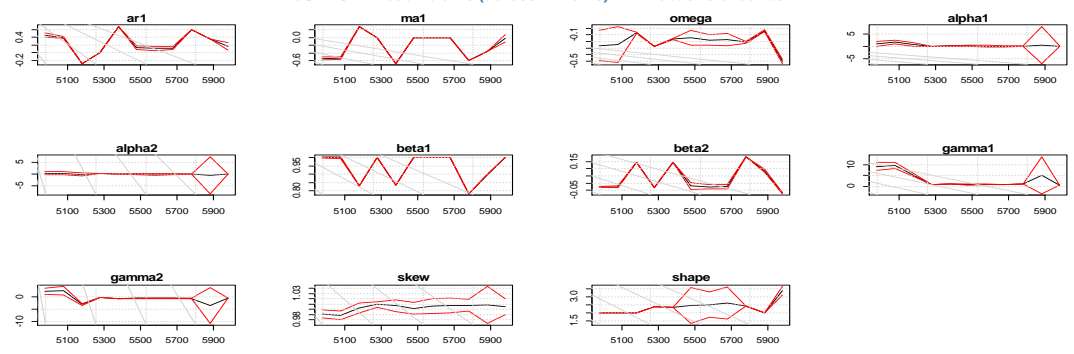

$\mathrm{V}$

Figure 11: Rolling Forecast Plots from ARMA $(1,1)$ eGARCH $(2,2)$ at $99 \%$ confidence level (with sstd)

Key:

i. Density Forecast

ii. Sigma Forecast

iii. Series Forecast

iv. VaR Forecast

v. Fit Coefficients (with s.e band)

sstd: skewed student $t$ distribution 


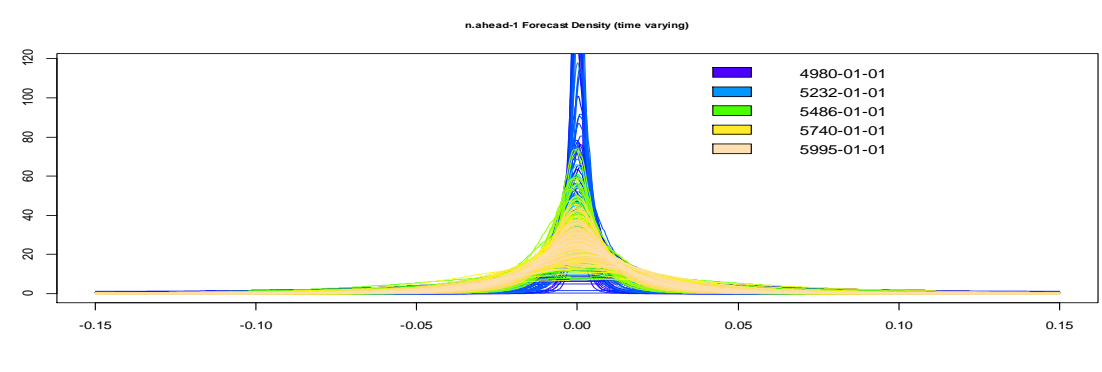

i

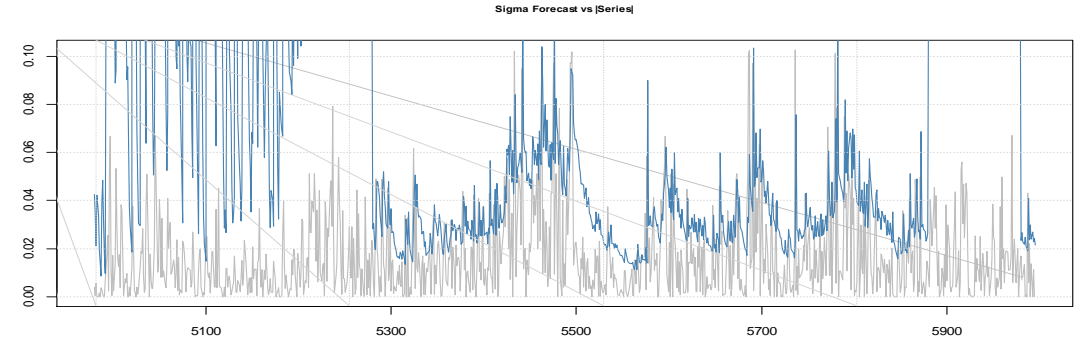

ii

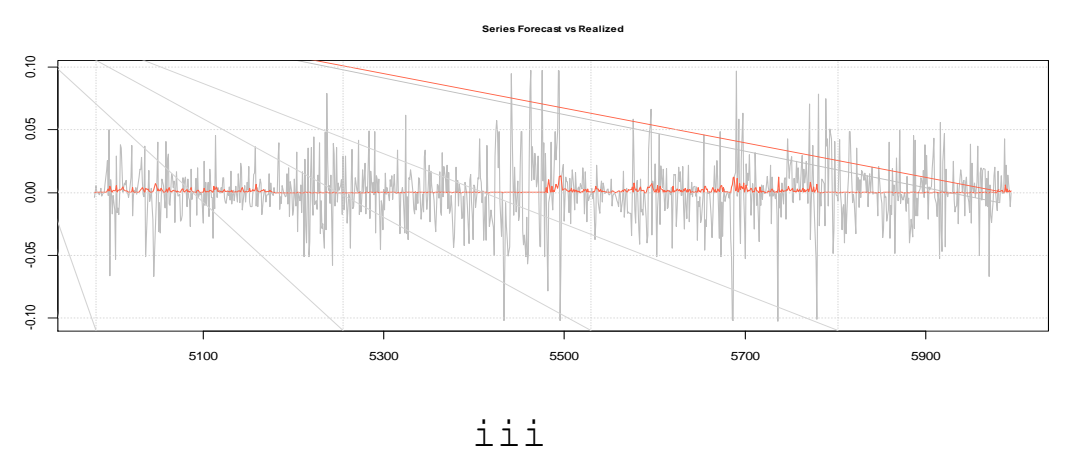

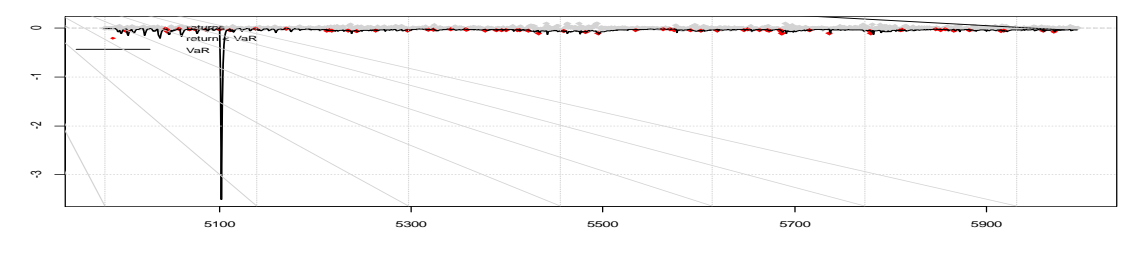

iv

eGARCH fit coefficients (across 11 refits) with robust s.e. bands
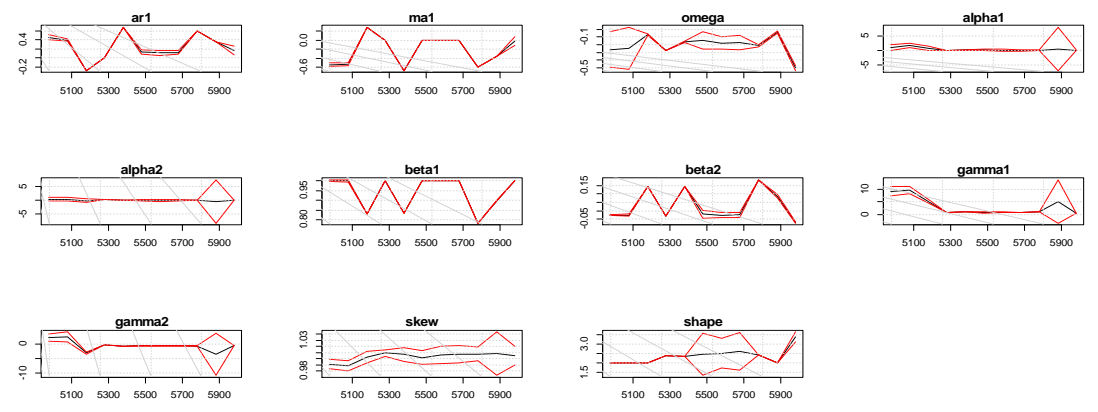

$\mathrm{V}$

Figure 12: Rolling Forecast Plots from $\operatorname{ARMA}(1,1)-$ eGARCH $(2,2)$ at 95\% confidence level (with sstd)

Key:

i. Density Forecast

ii. Sigma Forecast

iii. Series Forecast

iv. VaR Forecast

v. Fit Coefficients (with s.e band)

sstd: skewed student $t$ distribution 